\title{
NORMAL AND OUTLYING POPULATIONS OF THE MILKY WAY STELLAR HALO AT $[\mathrm{Fe} / \mathrm{H}]<-2^{*}$
}

\author{
Judith G. COHEN ${ }^{1}$, NORbert Christlieb ${ }^{2}$, IAN ThOMPSON ${ }^{3}$, ANDrew McWilliam ${ }^{3}$, \\ StePhen Shectman ${ }^{3}$, Dieter ReImers ${ }^{4}$, Lutz WisotzKi ${ }^{5}$, AND Evan Kirby ${ }^{6,7}$ \\ ${ }^{1}$ Palomar Observatory, Mail Stop 249-17, California Institute of Technology, Pasadena, CA 91125, USA; jlc@ astro.caltech.edu \\ ${ }^{2}$ Zentrum für Astronomie der Universität Heidelberg, Landessternwarte, Königstuhl 12, D-69117, Heidelberg, Germany; N.Christlieb@1sw.uni-heidelberg.de \\ ${ }^{3}$ Carnegie Observatories, 813 Santa Barbara Street, Pasadena, CA 91101, USA; \\ ian@obs.carnegiescience.edu, shec@obs.carnegiescience.edu, andy@obs.carnegiescience.edu \\ ${ }^{4}$ Hamburger Sternwarte, Gojenbergsweg 112, D-21029 Hamburg, Germany; dreimers@ @s.uni-hamburg.de \\ ${ }^{5}$ Leibniz-Institut für Astrophysik Potsdam, An der Sternwarte 16, D-14482 Potsdam, Germany; lutz@aip.de \\ ${ }^{6}$ University of California, Department of Physics and Astronomy, 4129 Frederick Reines Hall, Irvine, CA 92697, USA; ekirby@uci.edu \\ Received 2013 June 8; accepted 2013 September 23; published 2013 November 1
}

\begin{abstract}
From detailed abundance analysis of $>100$ Hamburg/ESO candidate extremely metal-poor (EMP) stars we find 45 with $[\mathrm{Fe} / \mathrm{H}]<-3.0$ dex. We identify a heretofore unidentified group: Ca-deficient stars with sub-solar $[\mathrm{Ca} / \mathrm{Fe}]$ ratios and the lowest neutron-capture abundances; the Ca-deficient group comprises $\sim 10 \%$ of the sample, excluding Carbon stars. Our radial velocity distribution shows that the carbon-enhanced stars with no $s$-process enhancements, CEMP-no, and which do not show $\mathrm{C}_{2}$ bands are not preferentially binary systems. Ignoring Carbon stars, approximately $15 \%$ of our sample are strong $(\geqslant 5 \sigma)$ outliers in one or more elements between $\mathrm{Mg}$ and $\mathrm{Ni}$; this rises to $\sim 19 \%$ if very strong $(\geqslant 10 \sigma)$ outliers for $\mathrm{Sr}$ and $\mathrm{Ba}$ are included. Examples include: HE0305-0554 with the lowest $[\mathrm{Ba} / \mathrm{H}]$ known; HE1012-1540 and HE2323-0256, two (non-velocity variable) C-rich stars with very strong $[\mathrm{Mg}, \mathrm{Al} / \mathrm{Fe}]$ enhancements; and HE1226-1149, an extremely $r$-process rich star.
\end{abstract}

Key words: Galaxy: abundances - Galaxy: formation - Galaxy: halo

Online-only material: color figures, machine-readable tables

\section{INTRODUCTION}

Extremely metal-poor (EMP) stars were presumably among the first stars formed in the Galaxy, and hence represent, in effect, a local high-redshift population. It is from this paradigm that the term "galactic archeology" arises. Such stars provide important clues to the chemical history of our Galaxy, the nature of early supernova (SN), the mode of star formation in the proto-Milky Way, and the formation of the Galactic halo. The number of EMP stars, i.e., those with $\mathrm{Fe}$ abundance ${ }^{8}[\mathrm{Fe} / \mathrm{H}]<-3.0$ dex, known as of 2005, is summarized by Beers \& Christlieb (2005); it has grown slowly since that time, but the sample is still quite small. The goal of the 0Z Survey, which began in 2001, is twofold: (1) to increase this sample substantially so that statistical studies of the Galactic EMP star population become feasible, and (2) to study at high spectral dispersion those new EMP stars we find to determine trends in abundance ratios, deduce implications regarding SN and nucleosynthesis in the young Milky Way, and derive constraints on how well the interstellar medium (ISM) in the forming Galaxy was mixed.

Our project, the 0Z Survey, focuses on candidate EMP stars from the Hamburg/ESO Survey (henceforth the HES; Wisotzki et al. 2000). These stars represent an in-situ probe of the Galactic halo. After completion of the Keck Pilot Project (Cohen et al. 2002; Carretta et al. 2002), we have published many papers over the past decade describing individual EMP stars, or small groups of such stars, of particular interest found in our survey,

\footnotetext{
* Based in part on observations obtained in part at the W. M. Keck Observatory, which is operated jointly by the California Institute of Technology, the University of California, and the National Aeronautics and Space Administration.

7 Southern California Center for Galaxy Evolution Fellow.

8 The standard nomenclature is adopted; the abundance of element $X$ is given by $\epsilon(X)=N(X) / N(H)$ on a scale where $N(H)=10^{12} \mathrm{H}$ atoms. Then $[X / H]=$ $\log _{10}[\mathrm{~N}(\mathrm{X}) / \mathrm{N}(\mathrm{H})]-\log _{10}[\mathrm{~N}(\mathrm{X}) / \mathrm{N}(\mathrm{H})]_{\odot}$, and similarly for $[\mathrm{X} / \mathrm{Fe}]$.
}

i.e., Cohen et al. (2003, 2004, 2005, 2006, 2007, 2008); those papers include a total of 74 individual stars. At this time, the $0 \mathrm{Z}$ project has ended, and we now have 122 candidate EMP stars mostly from the HES with high resolution spectra, many of which have not appeared in any of our earlier publications. This is the largest sample of EMP candidates with high dispersion spectra from a single survey analyzed by a single person. It is substantially larger than that of the First Stars Survey at the Very Large Telescope (VLT) led by R. Cayrel (see, e.g., Cayrel et al. 2004). A large component of the sample of EMP stars discussed by Yong et al. (2013) is in fact from our earlier publications.

In this paper, we focus on the $0 \mathrm{Z}$ sample as a whole, and in particular on the issue of outliers in various properties related to the chemical inventory of these stars. After defining the sample in Section 2, we give details of our analysis procedures in the next three sections, with Section 6.3 discussing the use of model atmospheres tailored to the individual abundance distribution within a specific star and Section 7 reporting on the overall quite good comparison for stars with independent abundance determinations by multiple groups.

Discussion of the outliers begins in Section 8, with linear fits to abundance ratios presented in Section 8.1. The largest family of outliers, the stars with sub-solar $[\mathrm{Ca} / \mathrm{Fe}]$, is presented in Section 8.2, with the next section discussing the other smaller outlier families. Carbon-rich EMP stars are discussed in Section 9; a presentation of $r$-process rich stars follows. Distance effects are examined in Section 11. Comments on nucleosynthesis and related issues are given in Section 12, and a brief summary concludes the paper.

\section{HIGH SPECTRAL RESOLUTION SAMPLE}

Christlieb et al. (2008) describe the procedure for selection of EMP candidates suspected to have $[\mathrm{Fe} / \mathrm{H}]<-3.0$ dex from the database of digitized spectra of the Hamburg/ESO Survey 
(Wisotzki et al. 2000), an objective prism survey whose primary goal was to find bright quasars. The original EMP candidate lists were generated from the HES database by N. Christlieb and a subset of these were transmitted to the Caltech and Carnegie 0Z core project members in 2001 August. As will be discussed in N. Christlieb et al. (2013, in preparation), because the fraction of higher-metallicity interlopers was unknown, but feared to be substantial, these candidates were followed up with moderate resolution spectroscopy using the Double Spectrograph (Oke \& Gunn 1982) at the Hale Telescope or the Boller and Chivens spectrograph at the $6 \mathrm{~m}$ Magellan Clay Telescope. Approximately 1540 candidates were observed. From each moderate resolution spectrum, a metallicity denoted $[\mathrm{Fe} / \mathrm{H}](\mathrm{HES})$ was generated using a variant of the algorithm of Beers et al. (1998), which relies on the strength of $\mathrm{H} \delta$ as an estimator of $T_{\text {eff }}$. The strength of the $3933 \AA$ Ca II absorption line then provides an estimate of the metallicity. The mod-res follow up program and results will be discussed in detail in $\mathrm{N}$. Christlieb et al. (2013, in preparation).

The sample selected for high dispersion observations from our mod-res follow up spectra of HES EMP candidates consisted of those stars with the lowest values of $[\mathrm{Fe} / \mathrm{H}](\mathrm{HES})$. Observations for the sample discussed here are primarily from HIRES (Vogt et al. 1994) at the Keck I Telescope with a substantial contribution of spectra from MIKE (Bernstein et al. 2003) at the Magellan II Clay Telescope. The stellar sample for the Keck Pilot Project (Cohen et al. 2002; Carretta et al. 2002; Lucatello et al. 2003) was assembled by N. Christlieb. It consisted of candidates with mod-res spectroscopy from other telescopes, which were subsequently observed with HIRES in 2000 September. N. Christlieb and T. Beers also contributed some of the candidates for the 2001 observing season at HIRES since at that time our $0 \mathrm{Z}$ project had not yet built up its own set of candidates vetted by moderate resolution spectroscopy. MIKE/Magellan observations for the $0 \mathrm{Z}$ project did not begin until 2003, by which time our 0Z project had built up its own set of vetted candidate EMP stars.

As high dispersion observations and preliminary detailed abundance analyses proceeded over the years, it became clear that there was a problem with the Beers et al. (1998) algorithm in that it underestimated $[\mathrm{Fe} / \mathrm{H}]$ for extremely C-rich stars (Cohen et al. 2005). In later versions of this code, no value was assigned to $[\mathrm{Fe} / \mathrm{H}](\mathrm{HES})$ for such stars. Because it took a few years to isolate this bias and to understand its origin, which is discussed in detail in Cohen et al. (2005), such C-rich stars are over-represented in our high resolution sample. Furthermore, as the metallicity assignment algorithm evolved and hopefully improved with time, the sample of stars of most interest, i.e., those with $[\mathrm{Fe} / \mathrm{H}](\mathrm{HES})<-3.0 \mathrm{dex}$, also evolved, with more stars being added, and a few dropping out. Major reviews, merging of the DBSP/Palomar and B\&C/Magellan lists, and summaries of all the moderate resolution results were carried out in 2005 and in 2007 to inform subsequent high resolution observations.

\section{HIGH DISPERSION OBSERVATIONS OF EMP CANDIDATES}

HIRES observations commenced in 2000 September at the Keck I Telescope and continued at a rapid pace through late 2007 , extending at a slower pace until early 2012. The initial observations used the original $2048 \times 2048$ pixel CCD detector at spectral resolution $36,000(1.1 \times 7$ arcsec slit $)$ with 4.2 pixels per spectral resolution element. Because of the limited size of the detector, the spectral coverage was restricted to 3805 to $5325 \AA$. This instrumental configuration was adopted since good coverage at blue wavelengths is crucial given the overall weakness of the absorption lines in EMP candidates and the dearth of red absorption lines. In the late spring of 2004, the HIRES detector was upgraded to a mosaic of three CCDs optimized for the blue, green, and red spectral regions, respectively. This enabled wider spectral coverage with smaller pixels (6.7 per spectral resolution element) and with lower readout noise. The spectra taken after this are divided into two classes; those that used the blue HIRES cross disperser had spectral coverage from about 3250 to $5990 \AA$ A. Most subsequent observations used the red HIRES cross disperser with spectral coverage from 3880 to $8360 \AA$ A. There are small gaps between the three CCDs that form the mosaic, sometimes resulting in the loss of part or in a few cases all of a single echelle order. Initially the HIRES observations were reduced using echelle routines written by J. McCarthy (McCarthy 1988) within the Figaro package (Shortridge 1993). Beginning in 2003, we used the pipeline package $\mathrm{MAKEE}^{9}$ for this purpose.

Most of the HIRES observations from 2010 to 2012 were repeat observations of stars whose prior high dispersion spectrum either had a signal-to-noise ratio $(\mathrm{S} / \mathrm{N})$ lower than desired or whose wavelength coverage needed to be extended further to the red for the reasons to be discussed in Section 8.

MIKE observations began in 2003 and continued through 2008. Although there were a number of detector upgrades on the blue and red arms during this period, the pixel size and format of the detectors remained unchanged; only the performance of the detectors was improved. MIKE observations have a spectral resolution of 42,000 in the blue camera and 32,000 in the red camera (corresponding to a 0.7 arcsec wide slit) with full spectral coverage, and are Nyquist sampled, with (typically) 2 pixels per spectral resolution element. The MIKE pipeline of Kelson (2003) was used to reduce the spectra.

The total sample of EMP candidates with high dispersion spectra and detailed abundance analyses presented here, including those from the Keck Pilot Project (Cohen et al. 2002; Carretta et al. 2002) and a few stars selected from the HK Survey (Beers et al. 1985, 1992), is 122 stars. The sample and a log of the HIRES/Keck and Mike/Magellan observations are presented in Table 1. The total integration time for this set of spectra is $\sim 210 \mathrm{hr}$.

The $\mathrm{S} / \mathrm{N}$ per spectral resolution element in the continuum at the center of the order near $5300 \AA$ is given in Table 1 . The spectral resolution is taken as 36,000 (that of a 1.1 arcsec wide slit) for HIRES. For MIKE, the 0.7 arcsec wide slit corresponds to 2 pixels at the detectors, to a spectral resolution of $42,000(32,000)$ in the blue (red) camera. ${ }^{10}$ For HIRES spectra, the $\mathrm{S} / \mathrm{N}$ is calculated from the observed detected electrons/spectral pixel in the final one-dimensional (1D) spectra (DN), ignoring such issues as cosmic rays, the contribution of sky background to the noise, etc. We have checked that this "quick and dirty" value (i.e., $\mathrm{S} / \mathrm{N}$ (quick) = $\left.\sqrt{\mathrm{DN} \times\left(e^{-} / \mathrm{DN}\right)}\right)$ provides an accurate determination of $\mathrm{S} / \mathrm{N}$ by selecting line-free regions approximately $30 \AA$ wide in the spectra of the most metal-poor stars and calculating the dispersion in DN across these intervals in segments of 20 pixels

\footnotetext{
9 MAKEE was developed by T.A. Barlow specifically for reduction of Keck HIRES data. It is freely available on the world wide web at the Keck Observatory home page, http://www2.keck.hawaii.edu/inst/hires.

10 A small number of MIKE and HIRES spectra were taken at even higher spectral resolution, but the $\mathrm{S} / \mathrm{N}$ are quoted for the values given above.
} 
Table 1

Sample of Candidate EMP Stars, Mostly from the HES

\begin{tabular}{|c|c|c|c|c|c|c|c|c|}
\hline ID & $\begin{array}{l}\text { R.A. } \\
(2000)\end{array}$ & $\begin{array}{c}\text { Decl. } \\
(2000)\end{array}$ & $\begin{array}{c}V \\
(\mathrm{mag})\end{array}$ & Date & $\begin{array}{c}t(\exp ) \\
(\mathrm{sec})\end{array}$ & $\mathrm{S} / \mathrm{N}^{\mathrm{a}}$ & Spec Mode ${ }^{b}$ & $\begin{array}{c}v_{r}{ }^{\mathrm{c}} \\
\left(\mathrm{km} \mathrm{s}^{-1}\right)\end{array}$ \\
\hline HE0007-1832 & 000952.8 & -181612 & 15.46 & 2001 Sep & 8400 & 90 & $\mathrm{O}$ & 26.4 \\
\hline HE0009-6039 & 001131.0 & -602221 & 16.54 & 2004 Sep & 1800 & 35 & M & 25.0 \\
\hline HE0011-0035 & 001415.8 & -001859 & 15.04 & 2001 Sep & 9600 & 100 & $\mathrm{O}$ & -170.6 \\
\hline HE0012-1441 & 001527.1 & -142437 & 16.40 & 2002 May & 10,800 & 80 & $\mathrm{O}$ & 11.2 \\
\hline HE0017-4346 & 002016.2 & -433018 & 15.88 & 2004 Sep & 1800 & 57 & M & 58.9 \\
\hline
\end{tabular}

Notes.

a $\mathrm{S} / \mathrm{N}$ per spectral resolution element in the continuum near $5300 \AA$ A. (HIRES: spectral resolution 36,000. MIKE 42,000 blue camera, 32,000 red camera).

${ }^{b}$ Spectrograph mode: $\mathrm{O}=$ HIRES prior to detector upgrade, $\mathrm{B}=$ HIRES-B after upgrade, $\mathrm{R}=\mathrm{HIRES}-\mathrm{R}$ after upgrade, $\mathrm{M}=\mathrm{Mike} / \mathrm{Magellan}$.

${ }^{\mathrm{c}}$ Heliocentric radial velocity.

${ }^{\mathrm{d}} V$ mag adopted from the HES in the absence of a better source.

(This table is available in its entirety in a machine-readable form in the online journal. A portion is shown here for guidance regarding its form and content.)

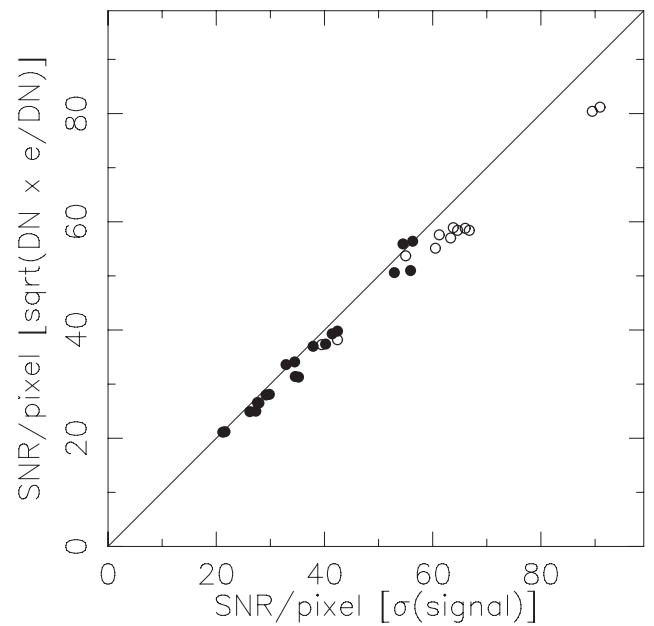

Figure 1. Comparison of $\mathrm{S} / \mathrm{N}$ calculated by looking at the dispersion in DN within line-free regions of spectra of the most metal-poor stars (horizontal axis) vs. that calculated in a simplified way (vertical axis) shows good agreement between the two measures. Filled circles are from HIRES-B spectra, open circles from HIRES-R spectra, all using the green or red CCDs of the mosaic HIRES detector.

length. The short length removes any curvature in the continuum arising from the use of an echelle grating. We then use the mean of the resulting $\sigma$ values to determine a more realistic value for $\mathrm{S} / \mathrm{N}$ without making any simplifying assumptions. This is compared with the $\mathrm{S} / \mathrm{N}$ (quick) in Figure 1. The agreement, shown for 32 test cases using the mosaic HIRES detector, is extremely good. The slight drop below equality seen in Figure 1, most obvious at the highest DN levels, may be due to the limited precision of the conversion factor $\mathrm{e}^{-} / \mathrm{DN}$ in the HIRES online documentation.

\section{EQUIVALENT WIDTHS AND RADIAL VELOCITIES}

The pipeline MAKEE when applied to HIRES spectra outputs a 1D spectrum for each echelle order whose wavelength scale has the heliocentric velocity correction applied. For the MIKE spectra, the heliocentric corrections were applied later.

Equivalent widths were automatically measured from the 1D spectra using the code EWDET2 which is based on the code EWDET described in Ramírez et al. (2001). This code carries out a continuum fit with an algorithm similar to that used in the IRAF script "continuum," then searches for absorption features. For each feature judged to be real, the continuum fit at that wavelength is evaluated followed by a Gaussian fit to the absorption feature. The output is a list of the central wavelength, equivalent width, and half width of each feature, and an estimate of the uncertainty in the equivalent width. Note that this list, together with a list of laboratory lines, is also used to determine the radial velocity of the star. EWDET2 works well on the HIRES spectra, but is somewhat less reliable for the MIKE spectra, where the spectra are Nyquist sampled. The smaller number of pixels per spectral resolution element in the MIKE spectra makes the Gaussian fitting routine used by EWDET2 sometimes output spurious results.

Extensive hand checking of equivalent widths had to be done for the MIKE spectra. For the HIRES spectra, some hand checking was done particularly to add lines too weak for the automatic routine to find. $W_{\lambda}$ for the strongest lines were always checked by hand to ensure that the full extent of the damped wings was included.

A master line list of features we adopted for radial velocity determination was constructed. It contains 38 reasonably isolated strong features from 3815 to $6643 \AA$ that one might expect to be present and reasonably strong even in EMP stars. The laboratory wavelengths for these features were taken from the NIST database as of 2002. Lines falling within the strongest $\mathrm{CH}$ and $\mathrm{CN}$ bands are omitted if the star under consideration is C-rich.

An initial guess of the radial velocity was made by searching the EWDET2 output by hand for the Mg triplet lines. Then a code searches for these 38 features within the list of features found by EWDET2, rejecting observed lines with $W_{\lambda}<50 \mathrm{~m} \AA$, and calculates a radial velocity for each of the laboratory lines. The individual $v_{r}$ values for lines which occur in two different echelle orders, and hence have independent measurements, are averaged. Finally an average $v_{r}$ and an rms dispersion are calculated for the subset of the 38 lab lines that are actually detected in a given spectrum. This is repeated after a $3 \sigma$ clip in $v_{r}$ to determine the adopted $v_{r}$ for each star. At most one laboratory line is eliminated by applying this $3 \sigma$ clip.

The master line list also contains a list of small corrections in wavelength for each individual line used that was developed in late 2001 by comparing the line-by-line $v_{r}$ measurements of a reasonable number of stars. Most of them correspond to less than $1 \mathrm{~km} \mathrm{~s}^{-1}$. This correction file has been used throughout. After the HIRES upgrade, additional lines were added to the master list at redder wavelengths to form the final list of 
38 lines. All the added lines are redder than the $\mathrm{Mg}$ triplet and have their correction value set to zero.

For spectra from HIRES taken after the 2004 detector upgrade with reasonable $\mathrm{S} / \mathrm{N}$ of stars which are not highly enhanced in carbon, this procedure gives an rms dispersion for the set of $v_{r}$ lines around the mean $v_{r}$ of less than $0.15 \mathrm{~km} \mathrm{~s}^{-1}$. Fewer lines from the master list for $v_{r}$ determinations can be picked up in the spectra of the cool C-rich stars, and there may be some blending with molecular features. Hence the dispersion around the final $v_{r}$ is often larger for C-rich stars, sometimes reaching as much as $1.5 \mathrm{~km} \mathrm{~s}^{-1}$. The uncertainty in the adopted $v_{r}$ is then nominally $\sigma / \sqrt{N(\text { lines })}$.

However, there may be a systematic contribution to the uncertainty in $v_{r}$ arising from placement of the star within the slit. If the slit is "wide" compared to the seeing, this becomes a concern. The worst case among our high resolution spectra is that of HIRES, where a 1.1 arcsec slit was used for essentially all of the spectra, in an attempt to avoid light loss while maintaining a sufficiently high spectral dispersion. However, when the seeing is very good (i.e., 0.6 arcsec or less), one is centering the star within the slit with only a few percent of the total light of the star not going through the slit. If guiding is done using another star within the guider field once the object of interest is placed within the spectrograph slit, the location of the star within the slit should be preserved throughout the exposure assuming perfect correction for field rotation. Thus centering within the slit may thus be slightly different from one star to another.

Simulations suggest that a 0.1 arcsec difference in placing the star within the width of the slit near its center will produce a shift in measured $v_{r}$ of less than $0.6 \mathrm{~km} \mathrm{~s}^{-1}$. We therefore assume that all HIRES spectra have a $0.6 \mathrm{~km} \mathrm{~s}^{-1}$ contribution to the total $v_{r}$ uncertainty. MIKE spectra use narrower slits, so this value should be a firm upper limit to the $v_{r}$ offset due to placement of the star in the slit.

In addition, we did not take other steps necessary to secure the highest possible radial velocity accuracy from HIRES. The configuration (usually the setting of the cross disperser) of HIRES varied from run to run depending on the goals for the HES and for whatever other observations were planned. To test the $v_{r}$ accuracy we actually achieved, we used the terrestrial atmospheric band near $6860 \AA$. The measured wavelengths of selected isolated lines in this band should be constant in the final 1D spectra, but they are not. They show differences from run to run corresponding to a maximum of $3 \mathrm{~km} \mathrm{~s}^{-1}$, although the variations within a given run (i.e., with a fixed HIRES configuration) were generally closer to $1 \mathrm{~km} \mathrm{~s}^{-1}$. We emphasize that these variations most likely do not arise in the spectrograph itself, but rather from the idiosyncrasies of wavelength fitting within the reduction pipeline. The largest apparent wavelength shifts occurred when this atmospheric band shifted from the first order of the red detector in the three CCD mosaic to the last order of the green detector, with one or both being only partial orders due to gaps between the CCDs in the mosaic.

G. W. Preston (2001, private communication) kindly supplied a compilation of his monitoring of metal-poor red giants using the echelle spectrograph at the DuPont $2.5 \mathrm{~m}$ Telescope of the Las Campanas Observatory. Those with eight or more independent measurements which had a low rms dispersion for the set of measured $v_{r}$ (typically less than $0.5 \mathrm{~km} \mathrm{~s}^{-1}$ ) were adopted by our $0 Z$ Project as radial velocity standard stars. One or more of these stars was observed during each HIRES run for the first $4 \mathrm{yr}$ of the $0 \mathrm{Z}$ project. With one exception, all our observations of stars from Preston's list agree with his $v_{r}$ to within the observational uncertainties.
Table 2

$v_{r}$ for EMP Stars with Multiple Spectra from HIRES

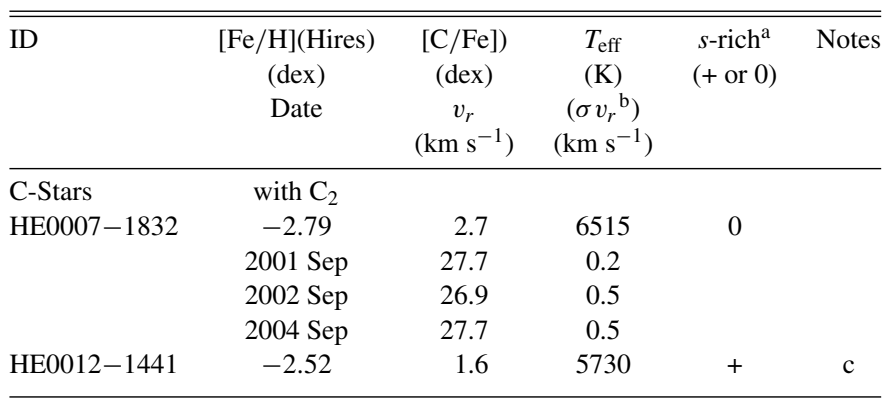

Notes.

${ }^{\text {a }}$ High $[\mathrm{Ba} / \mathrm{Fe}](+)$ versus normal $[\mathrm{Ba} / \mathrm{Fe}](0)$.

${ }^{\mathrm{b}}$ Uncertainty in the mean $v_{r}$ computed from the rms dispersion.

c Spectrum shows double lines.

${ }^{\mathrm{d}}$ Lucatello et al. (2003) gives an orbit for this close binary with period 3.14 days and with full amplitude of $v_{r}$ of $104 \mathrm{~km} \mathrm{~s}^{-1}$.

${ }^{\mathrm{e}} \mathrm{HE} 0058-0244$ is a rediscovery of CS22183-015, see Johnson \& Bolte (2002) and Tsangarides et al. (2004).

${ }^{f}$ MIKE/Magellan spectrum.

g J. Andersen et al. (2013, in preparation) found this star to be a long period binary.

h J. Andersen et al. (2013, in preparation) have determined a period of 289 days for this binary.

i A spectrum with low $\mathrm{S} / \mathrm{N}$.

${ }^{\mathrm{j}}$ HE2356-0410 is a rediscovery of CS22957-27, previously known binary with $v_{r}$ range -62 to $-78 \mathrm{~km} \mathrm{~s}^{-1}$ (Preston \& Sneden 2001).

${ }^{\mathrm{k}} \mathrm{HE} 2344-2800$ is a rediscovery of CS22966-048.

${ }^{1} \mathrm{HE} 2323-0256$ is a rediscovery of CS22949-037.

$\mathrm{m}$ Double lined (Cohen et al. 2002).

(This table is available in its entirety in a machine-readable form in the online journal. A portion is shown here for guidance regarding its form and content.)

A significant fraction $(\sim 25 \%)$ of the stars in the $0 \mathrm{Z}$ sample have been observed more than once at high spectral dispersion during the course of the $0 \mathrm{Z}$ project, with a typical separation of several years; see Table 2. This offers a good opportunity to test for binarity. Gunn \& Griffin (1979) found that a typical $v_{r}$ jitter in metal-poor red giants due to atmospheric effects is $\sim 1 \mathrm{~km} \mathrm{~s}^{-1}$. Bearing this in mind and given the issues for $v_{r}$ discussed above, we assume that velocity differences between the various epochs of observation for a particular star exceeding $5 \mathrm{~km} \mathrm{~s}^{-1}$ imply that the star is a binary. Stars with multiple spectra whose velocity differences are less than $3 \mathrm{~km} \mathrm{~s}^{-1}$ are taken to be consistent with no variation in radial velocity and hence no evidence that the star is a member of a binary system, while stars with $3<\Delta\left(v_{r}\right)<5 \mathrm{~km} \mathrm{~s}^{-1}$ are assigned as possible binaries. Previously known binaries are indicated in Table 2. This table includes a few shorter HIRES exposures, not listed in Table 1, acquired only to check $v_{r}$, as well as $v_{r}$ measurements from the literature for our sample stars when available from other high dispersion studies.

We find (see Table 2) that seven of the total of eight C-stars (i.e., C-rich stars with detectable $\mathrm{C}_{2}$ bands) in this table with high $[\mathrm{Ba} / \mathrm{Fe}]$ are binaries or probable binaries as defined above. The high fraction of binaries is in agreement with an earlier study by Lucatello et al. (2005) for a sample of comparable size of C-rich Ba-rich metal-poor stars. This is not surprising given that the standard explanation of $s$-process enrichment is mass transfer in a system where the primary has gone through the asymptotic giant branch (AGB), see, e.g., Busso et al. (1999). Two of the four $\mathrm{C}$-stars with normal $[\mathrm{Ba} / \mathrm{Fe}]$ are confirmed binaries with 
periods, one (a hot dwarf with three epochs of HIRES spectra) does not appear to be a binary, but might now be or have been one, and the fourth has only one epoch of observation. Thus it appears that even the $\mathrm{C}$-stars with normal $[\mathrm{Ba} / \mathrm{Fe}]$ are mostly binaries.

The picture changes for the five C-rich stars that do not show $\mathrm{C}_{2}$ bands. (Note that the taxonomy and characteristics of C-rich stars are discussed in Section 9.) The only one with high $[\mathrm{Ba} / \mathrm{Fe}]$ (the CEMP-rs star HE2148-1247) appears to be a binary. One of the other four with normal $[\mathrm{Ba} / \mathrm{Fe}]$ may be a binary, but there is no evidence to support binarity for the remaining three stars.

Of the $18 \mathrm{C}$-normal stars with multiple $v_{r}$ determinations, 3 are definite binaries and 3 more are probable binaries, i.e., a minimum binary fraction of $17 \pm 9 \%$, possibly being as large as $33 \%$. The minimum estimated binary fraction for our sample of EMP stars is consistent with that reported by Latham et al. (2002), who present the results of an extensive radial velocity monitoring program for a large sample of proper motion stars carried out over a time span of $18 \mathrm{yr}$. They find that $15 \%$ of halo field stars in the solar neighborhood are binaries, and that this fraction is identical to within the uncertainties to that of a similar sized sample of Galactic disk stars.

In order to investigate more quantitatively whether the radial velocity distributions differ between $\mathrm{C}$-normal, $\mathrm{C}$-rich with $\mathrm{C}_{2}$ bands, and $\mathrm{C}$-rich without $\mathrm{C}_{2}$ bands, we computed the velocity range, $\Delta v_{r}=v_{\max }-v_{\min }$, for each star with multiple epoch spectra. For the 18 stars in the C-normal group, $\Delta v_{r}$ ranges from 0.2 to $5.5 \mathrm{~km} \mathrm{~s}^{-1}$. For the group of $11 \mathrm{C}$-rich stars with $\mathrm{C}_{2}$ bands, there are 4 with $\Delta v_{r}$ above $10 \mathrm{~km} \mathrm{~s}^{-1}$ and another at $6.8 \mathrm{~km} \mathrm{~s}^{-1}$, all well above the range for the $\mathrm{C}$-normal group. The five $\mathrm{C}$-rich stars without $\mathrm{C}_{2}$ bands range from 0.8 to $5.7 \mathrm{~km} \mathrm{~s}^{-1}$, very similar to the $\mathrm{C}$-normal distribution of $\Delta v_{r}$ values. $\mathrm{A} \mathrm{K}-\mathrm{S}$ test comparing the velocities of the $\mathrm{C}$-normal and C-rich stars without $\mathrm{C}_{2}$ indicates a probability of only $0.3 \%$ that the two distributions differ. On the other hand, the $\mathrm{K}-\mathrm{S}$ test indicates an $80 \%$ probability that the velocities of the C-rich stars with $\mathrm{C}_{2}$ bands differ from the $\mathrm{C}$-normal velocities.

Thus, the velocities are consistent with the idea that most of the C-rich stars with $\mathrm{C}_{2}$ bands are binaries, while the binary fraction of the $\mathrm{C}$-rich stars without $\mathrm{C}_{2}$ is similar to the $\mathrm{C}$-normal stars. Except for HE2148-1247, our C-rich stars without $\mathrm{C}_{2}$ bands are not particularly enhanced in Ba: they are CEMP-no stars, i.e., without $s$-process enhancements.

As we noted in Cohen et al. (2006), the CEMP-no stars show very similar characteristics to population I early R-type carbon stars: both show carbon enhancements but normal $s$-process abundances (Dominy 1984), and both have low ${ }^{12} \mathrm{C} /{ }^{13} \mathrm{C}$ ratios. Here we find that the CEMP-no stars also have low binary fractions, qualitatively similar to the conclusion of McClure (1997) for R-type carbon stars.

Thus, our velocities lend further support to the idea that CEMP-no stars without $\mathrm{C}_{2}$ bands are the population II equivalent of the population I early R-type carbon stars.

\section{STELLAR PARAMETERS}

We follow the procedures developed earlier by J. Cohen and collaborators and described in Cohen \& Melendez (2005). We rely on infrared magnitudes $J$ and $K_{s}$ from the Two Micron All Sky Survey (2MASS; Skrutskie et al. 2006; Cutri et al. 2003) combined with optical photometry from a variety of sources, including our Andicam queue photometry program at CTIO for $V, I$ described in N. Christlieb et al. (2013, in preparation). If a
0Z sample star with a high resolution spectrum was not observed by Andicam or by the Sloan Digital Sky Survey (SDSS; York et al. 2000), we fell back on our small photometry program at the 40 inch Swope telescope at the Las Campanas Observatory in 2001, or, in the four cases where nothing else was available, relied on the HES $V$ mag. A comparison of the HES $V$ mags with those of higher precision from the SDSS will be given in N. Christlieb et al. (2013, in preparation); for 51 stars from the OZ Survey in common, the mean difference in $V$ (Andi) versus $V($ SDSS) (the conversion formulae of Smith et al. 2002 are used to go from SDSS gri to $V, I$ ) is $0.00 \mathrm{mag}$, while the rms deviation is $0.07 \mathrm{mag}$.

From these we form the colors $V-I, V-J$, and $V-K_{s}$. We match these using the predicted color grid of Houdashelt et al. (2000), which we have earlier shown to be essentially identical to those from the Kurucz (Kurucz 1993) and MARCS models (Gustaffson et al. 1975). We chose these specific colors to have good discrimination for effective temperature, $T_{\text {eff }}$ while minimizing their sensitivity to metallicity and surface gravity. The all-sky uniform 2MASS colors were extremely useful, but the faintest $0 \mathrm{Z}$ sample stars are approaching the faint limit for 2MASS; they have rather large uncertainties for their IR colors, particularly for $K_{s}$. We used the reddening maps of Schlegel et al. (1998) assuming that the EMP candidate was beyond the absorbing gas clouds, which are confined fairly tightly to the Galactic plane. The first pass at $T_{\text {eff }}$ used $[\mathrm{Fe} / \mathrm{H}](\mathrm{HES})$ as the stellar metallicity.

Once a first pass at $T_{\text {eff }}$ was made, we calculated the surface gravity, $\log (g)$, by combining a bolometric correction adopted from the Houdashelt et al. (2000) color grid with an $\alpha$-enhanced EMP isochrone from Kim et al. (2002) and Yi et al. (2003) and dereddened $V$ mag. We assume a fixed stellar mass of $0.8 M_{\odot}$. We then repeated this procedure using the $\log (g)$ from the first iteration and the $[\mathrm{Fe} / \mathrm{H}]$ from the first pass abundance analysis to obtain the nominal stellar parameters. Note that these are set without any reference to the spectral features, except for a rough value of $[\mathrm{Fe} / \mathrm{H}]$, which is not crucial over the range relevant here, since at such low metallicities the contribution of atomic lines to the total opacity is not significant and at these low $[\mathrm{Fe} / \mathrm{H}]$ the electron density is dominated by ionization of $\mathrm{H}$, not of the metals.

Table 3 lists the nominal and adopted stellar parameters for our sample, with the final $[\mathrm{Fe} / \mathrm{H}]$ values from our detailed abundance analyses in the last column. The uncertainty in $T_{\text {eff }}$ is taken as the rms $\sigma$ over the three colors we use to determine $T_{\text {eff }}$. Stars with $T_{\text {eff }}$ near that of the main sequence turnoff can be either above it (i.e., subgiants) or lower luminosity dwarfs. In the absence of a distance, distinguishing between these two choices can only be made by analyzing the spectra, i.e., by the Fe ionization equilibrium. Our $0 \mathrm{Z}$ high dispersion sample is shown in the $T_{\text {eff }}-\log (g)$ plane in Figure 2.

There are some stars with very large uncertainties in their nominal $T_{\text {eff }}$ (see the third column of Table 3 ). In early 2012, in an effort to understand the origin of these few very large $\sigma$, those stars with $\sigma\left(T_{\text {eff }}\right)>200 \mathrm{~K}$ were searched for in DR8 of the SDSS (Aihara et al. 2011). Several were located, but the SDSS photometry, transformed into $V$ and $I$ using the equations of Smith et al. (2002), was in all cases very close to that from Andicam. Most of the high $\sigma\left(T_{\text {eff }}\right)$ stars are C-stars; presumably the strong $\mathrm{CH}, \mathrm{CN}$, and $\mathrm{C}_{2}$ molecular bands are distorting the colors. The few other stars with high $\sigma\left(T_{\text {eff }}\right)$ are at the faint end of the 2MASS sample with large uncertainties at $K_{s}$. There are only four stars for which we had to rely on $V(\mathrm{HES})$ as no 
Table 3

Nominal and Adopted Stellar Parameters

\begin{tabular}{|c|c|c|c|c|c|c|c|}
\hline \multirow[t]{2}{*}{ ID } & \multicolumn{3}{|c|}{ Nominal $^{\mathrm{a}}$} & \multicolumn{3}{|c|}{ Adopted } & \multirow{2}{*}{$\begin{array}{c}{[\mathrm{Fe} / \mathrm{H}]^{\mathrm{c}}} \\
(\mathrm{dex})\end{array}$} \\
\hline & $\begin{array}{l}T_{\text {eff }} \\
(\mathrm{K})\end{array}$ & $\begin{array}{c}\sigma\left(T_{\text {eff }}\right)^{\mathrm{b}} \\
(\mathrm{K})\end{array}$ & $\begin{array}{c}\log (g) \\
(\operatorname{dex})\end{array}$ & $\begin{array}{l}T_{\text {eff }} \\
(\mathrm{K})\end{array}$ & $\begin{array}{c}\log (g) \\
(\operatorname{dex})\end{array}$ & $\begin{array}{c}v_{t} \\
\left(\mathrm{~km} \mathrm{~s}^{-1}\right)\end{array}$ & \\
\hline HE0007-1832 & 6500 & 30 & 3.8 & 6500 & 3.8 & 1.7 & -2.79 \\
\hline HE0009-6039 & 5270 & 150 & 2.7 & 5250 & 2.7 & 1.8 & -3.29 \\
\hline HE0011-0035 & 4955 & 25 & 1.9 & 4950 & 1.8 & 2.0 & -2.99 \\
\hline HE0012-1441 & 5610 & 155 & 3.3 & 5750 & 3.5 & 1.6 & -2.52 \\
\hline HE0017-4346 & 6198 & 53 & 3.8 & 6198 & 3.8 & 1.2 & -3.07 \\
\hline
\end{tabular}

Notes.

a The nominal $T_{\text {eff }}$ and $\log (g)$ are based solely on the available photometry, specifically the mean of that deduced from each of $V-I, V-J, V-K$. There are two possible solutions for stars near the main sequence turnoff; the one adopted is given.

${ }^{\mathrm{b}}$ Dispersion about the mean for the values (maximum of three) of $T_{\mathrm{eff}}$.

c Value from our high-resolution analysis adopting $[\mathrm{Fe} / \mathrm{H}](\mathrm{Sun})=7.45 \mathrm{dex}$.

d See Keck Pilot Project analyses (Cohen et al. 2002; Carretta et al. 2002; Lucatello et al. 2003).

e The HIRES spectrum of this star suggests that it is on the horizontal branch, not the RGB.

(This table is available in its entirety in a machine-readable form in the online journal. A portion is shown here for guidance regarding its form and content.)

accurate $V$ could be located nor were these four stars included in our own photometry programs, but these are not among those with unusually large $\sigma\left(T_{\text {eff }}\right)$. In a few other cases the optical photometry may not be as accurate as claimed. However, as can be seen in Table 3 , the dispersion around the nominal $T_{\text {eff }}$ of the three contributing colors is not unreasonably large for most stars and is in most cases consistent with the uncertainties of each color from their original sources. The quartiles in the distribution of dispersion in $T_{\text {eff }}$ from the three colors are 42 , 67 , and $133 \mathrm{~K}$, and $68 \%$ (that of $1 \sigma$ for a Gaussian distribution) of the total set of dispersions is less than $100 \mathrm{~K}$ and $94 \%$ (equivalent to $2 \sigma$ for a Gaussian distribution) is less than $200 \mathrm{~K}$. Only $13 \%$ have $\sigma\left(T_{\text {eff }}\right)>150 \mathrm{~K}$. Thus adopting $100 \mathrm{~K}$ as our nominal $T_{\text {eff }}$ uncertainty seems appropriate. With this $T_{\text {eff }}$ uncertainty, the slope of the red giant branch (RGB) in low metallicity isochrones from Yi et al. (2003) suggests 0.25 dex as the appropriate uncertainty in $\log (g)$.

\section{ABUNDANCE ANALYSIS PROCEDURES}

Our detailed abundance analyses begin with the stellar parameters derived as described above. We use plane-parallel LTE model atmospheres from the grid of Kurucz (1993) with no convective overshooting. We use the abundance determination code MOOG (Sneden 1973) as of 2002. Recently, an improved version of MOOG was presented by Sobeck et al. (2011). This update incorporates a number of improvements that are important for EMP stars. In particular, it includes a better treatment of coherent isotropic scattering, which in the 2002 version is treated as pure absorption. This provides a proper treatment of Rayleigh scattering, which can become an important opacity source in the blue and UV in EMP stars. The difference in deduced abundances between MOOG-2002 and MOOG-2010SCAT is thus largest for the coolest EMP giants, and also is a function of the wavelength of the line, becoming significant only at $\lambda<4000 \AA$, as was pointed out by Sobeck et al. (2011). The 5 stars in the HES sample with $T_{\text {eff }}<4800 \mathrm{~K}$, as well as the 15 stars with $4800<T_{\text {eff }}<5120 \mathrm{~K}$ with spectra extending well below $4000 \AA$, were reanalyzed in early 2012 with the updated

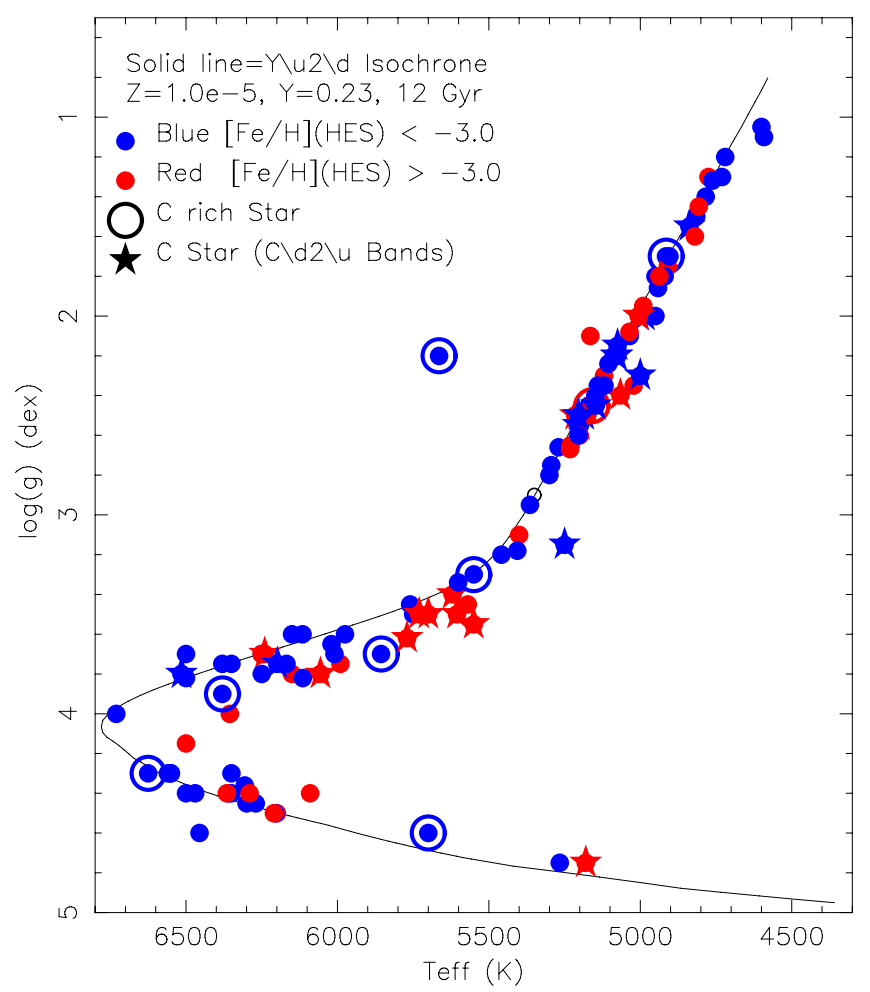

Figure 2. Adopted $T_{\text {eff }}$ vs. $\log (g)$ is shown for the 122 stars in the high resolution abundance sample studied here. There is one HB star. The colors denote the metallicity, while the symbols indicate the degree of C-enhancement; C-stars are shown by stars while $\mathrm{C}$-rich stars with no $\mathrm{C}_{2}$ are circled. The filled and open circles denote the metallicity.

(A color version of this figure is available in the online journal.)

code of Sobeck et al. (2011). These stars are indicated by the letter $\mathrm{S}$ after their ID in column 1 of Table 3. In general the changes were small; $v_{t}$ tended to be reduced by $0.1 \mathrm{~km} \mathrm{~s}^{-1}$, and the largest difference in abundance was a decrease of $0.3 \mathrm{dex}$ for the UV features in the coolest sample stars.

The determination of stellar parameters, measurement of equivalent widths, and detailed abundance analyses were all carried out by J. Cohen over the past decade, with the exception of 9 of the 11 stars $^{11}$ included in the Keck Pilot Project (Cohen et al. 2002; Carretta et al. 2002). During that time, the $g f$ values for several species have been updated. In particular, we are now using the current NIST (version 4.0; Ralchenko et al. 2010) values for $\mathrm{Mg}$ and $\mathrm{Ca}$, which are not the same as those we have used in the past. In late 2011, we therefore went through the abundance analyses of all the HES stars (except those from the Keck Pilot Project which were not subsequently reobserved) to homogenize the $g f$ values used for all the detected absorption lines in the abundance analyses of each of the sample stars to those on J. Cohen's current master list. The largest impact in deduced abundance was for $\mathrm{Mg}$ as the updates to the $g f$ values for several of the Mg I lines were large, and there are only a few detectable Mg I lines in EMP stars. Note that the $g f$ values for the $\mathrm{Mg}$ triplet lines did not change. The updated Mg I $g f$ values are clearly better; they significantly reduce the dispersion in $\mathrm{Mg}$ abundance determined from a typical set of $\mathrm{Mg}$ I lines. In addition to reducing $\sigma$, depending on which specific lines were detected in a particular star, a decrease in $[\mathrm{Mg} / \mathrm{H}]$ over our older values of up to 0.12 dex resulted.

\footnotetext{
11 Supplementary observations with HIRES were subsequently obtained by J. Cohen for 2 of the 11 stars included in the Keck Pilot Project and she then reanalyzed them.
} 


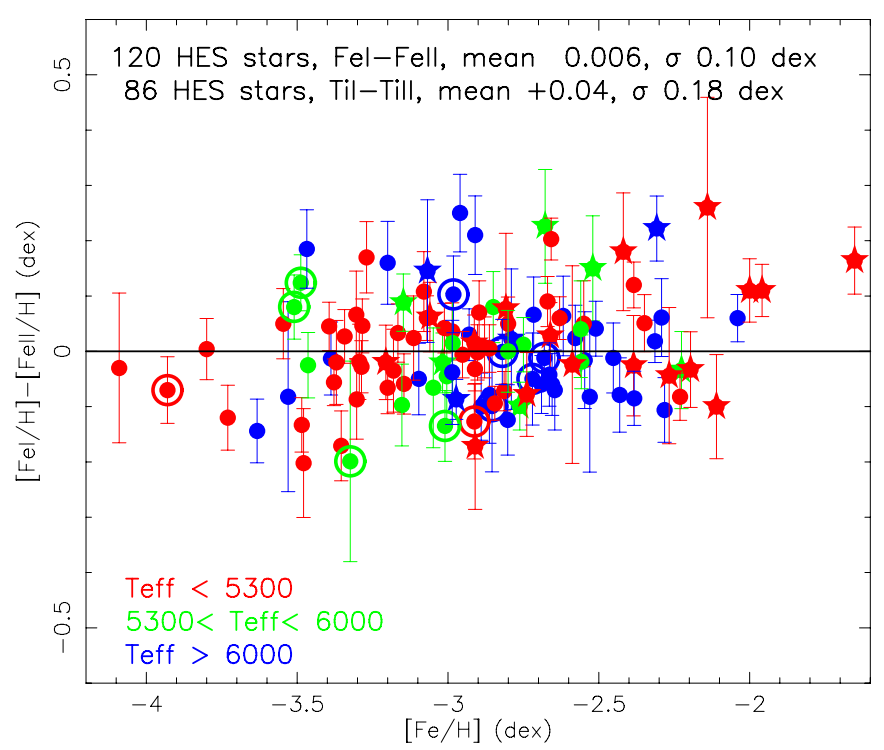

Figure 3. Ionization equilibrium for neutral vs. singly ionized $\mathrm{Fe}$ is shown as a function of $[\mathrm{Fe} / \mathrm{H}]$. Statistics for the $\mathrm{Fe}$ and $\mathrm{Ti}$ ionization equilibria are given in the text at the top of the figure. The symbol colors denote ranges of $T_{\text {eff }}$, red: $T_{\text {eff }}<5300 \mathrm{~K}$, green: $5300<T_{\text {eff }}<6000 \mathrm{~K}$, blue: $T_{\text {eff }}>6000 \mathrm{~K}$. The star symbols are $\mathrm{C}$-stars, while the circled points are $\mathrm{C}$-rich but do not show $\mathrm{C}_{2}$ bands. In the black and white version, the temperature ranges, from cooler to hotter, are indicated by open circles, filled gray circles, and black filled circles. This is the set of symbols used to define $T_{\text {eff }}$ and degree of $\mathrm{C}$ enhancement for the rest of the figures in this paper, with the exception of Figures 19 and 20.

(A color version of this figure is available in the online journal.)

The impact of our update of the Ca I $g f$ values was smaller as there are usually more detectable $\mathrm{Ca}$ I lines, only some of which had their transition probabilities changed. For a given star, smaller changes in $[\mathrm{Ca} / \mathrm{H}]$, ranging from +0.01 to +0.04 dex, occurred as a result of these changes.

Because of the uncertainty in the stellar parameters, we felt free to make small adjustments in the adopted $T_{\text {eff }}$ and $/$ or $\log (g)$ to improve the results of the abundance analyses (usually the ionization equilibrium) if necessary. These changes, which were always less than the dispersion in $T_{\text {eff }}$ values from the various available colors, are indicated in Table 3, where both the purely photometric stellar parameters and the final adopted values are given for our 122 star sample. The adopted stellar parameters of only 17 of the 122 sample stars differ from their photometric values by more than $20 \mathrm{~K}$ in $T_{\text {eff }}$ or more than $0.1 \mathrm{dex}$ in $\log (g)$.

Standard tests of the validity of our procedures include whether we were able to achieve good ionization equilibrium, and consistency of abundances from the set of Fe I lines observed in each star with $\chi, W_{\lambda}$, and $\lambda$. The results are very encouraging. For 120 stars (a few of the EMP stars have no detected Fe II lines), we find a mean difference between Fe abundance as determined from the neutral versus the ionized species of $0.00 \mathrm{dex}$, with $\sigma$ of only $0.10 \mathrm{dex}$. The adopted $T_{\text {eff }}$ uncertainty of $100 \mathrm{~K}$ corresponds to a shift in the ionization equilibrium between $\mathrm{Fe}$ I and $\mathrm{Fe}$ II of 0.09 dex, and the predicted $1 \sigma$ uncertainty in $[\mathrm{Fe} \mathrm{I} / \mathrm{Fe} \mathrm{II}]$ for giants including both stellar parameter and analysis uncertainties is 0.14 dex (see Table 4, with values for main sequence turnoff region stars in Table 5). The good agreement between these values suggests that our adopted uncertainty in $T_{\text {eff }}$ is reasonable. For 86 stars with both Ti I and Ti II detected, the mean difference between the deduced Ti abundance was only 0.04 dex, with a larger $\sigma$ of $0.18 \mathrm{dex}$; the corresponding entry in Table 4 is $\sigma=0.12$ dex, fairly close
Table 4

Abundance Ratio Changes for Small Changes in Stellar Parameters for Giants ${ }^{\mathrm{a}}$

\begin{tabular}{|c|c|c|c|c|c|c|}
\hline Species & $\begin{array}{c}\sigma[\mathrm{X} / \mathrm{Fe}]^{\mathrm{b}} \\
1 \mathrm{Star} \\
(1 \sigma)(\operatorname{dex})\end{array}$ & $\begin{array}{c}\Delta \log \epsilon(X) \\
T_{\text {eff }} \\
(\operatorname{dex})\end{array}$ & $\begin{array}{c}\Delta \log \epsilon(X) \\
\log (g) \\
(\operatorname{dex})\end{array}$ & $\begin{array}{c}\Delta \log \epsilon(X) \\
{[\mathrm{Fe} / \mathrm{H}]} \\
(\mathrm{dex})\end{array}$ & $\begin{array}{c}\Delta \log \epsilon(X) \\
v_{t} \\
(\operatorname{dex})\end{array}$ & $\begin{array}{c}\Delta \log \epsilon(X) \\
W_{\lambda} \\
(\operatorname{dex})\end{array}$ \\
\hline$[\mathrm{Fe} \mathrm{I} / \mathrm{H}]^{\mathrm{c}}$ & 0.17 & 0.11 & -0.02 & -0.04 & -0.05 & 0.01 \\
\hline$[\mathrm{Fe} \text { II } / \mathrm{H}]^{\mathrm{c}}$ & 0.10 & 0.00 & -0.08 & -0.03 & -0.03 & 0.04 \\
\hline$\left[\mathrm{Fe} \text { II } / \mathrm{Fe} \mathrm{I}_{\mathrm{I}}\right]^{\mathrm{d}}$ & 0.14 & 0.07 & 0.11 & 0.04 & 0.01 & 0.04 \\
\hline$\left[\mathrm{Ti}\right.$ II $/ \mathrm{Ti} \mathrm{I}^{\mathrm{d}}$ & 0.12 & 0.08 & -0.08 & -0.01 & -0.02 & 0.03 \\
\hline $\mathrm{C}(\mathrm{CH})$ & 0.13 & 0.11 & -0.04 & -0.04 & 0.00 & $0.05^{\mathrm{e}}$ \\
\hline $\mathrm{N}(\mathrm{NH})$ & 0.17 & 0.09 & -0.12 & 0.05 & -0.05 & $0.05^{\mathrm{e}}$ \\
\hline $\mathrm{Na} \mathrm{I}$ & 0.10 & 0.01 & -0.07 & -0.01 & 0.03 & 0.06 \\
\hline Mg I & 0.09 & -0.03 & 0.03 & -0.01 & 0.06 & 0.05 \\
\hline $\mathrm{Al} \mathrm{I}$ & 0.09 & -0.01 & 0.01 & 0.00 & 0.04 & 0.08 \\
\hline Si I & 0.16 & 0.00 & -0.05 & -0.02 & 0.11 & 0.11 \\
\hline $\mathrm{Ca} \mathrm{I}$ & 0.07 & -0.03 & 0.03 & 0.00 & 0.03 & 0.05 \\
\hline $\mathrm{Sc} \mathrm{II}^{\mathrm{f}}$ & 0.11 & 0.05 & -0.06 & 0.00 & 0.05 & 0.05 \\
\hline Ti I & 0.06 & 0.01 & 0.00 & 0.01 & 0.00 & 0.05 \\
\hline Ti II & 0.09 & 0.04 & -0.06 & 0.00 & 0.06 & 0.03 \\
\hline $\mathrm{Cr} \mathrm{I}$ & 0.07 & 0.01 & 0.01 & 0.00 & 0.05 & 0.05 \\
\hline $\operatorname{Mn} \mathrm{I}^{\mathrm{f}}$ & 0.09 & 0.04 & 0.00 & 0.00 & 0.03 & 0.08 \\
\hline Mn II UV f,g & 0.13 & 0.02 & -0.05 & -0.01 & -0.05 & 0.11 \\
\hline Fe II UV' & 0.22 & 0.04 & -0.06 & -0.02 & -0.17 & 0.11 \\
\hline $\mathrm{Co} \mathrm{I}^{\mathrm{f}}$ & 0.07 & 0.02 & -0.02 & 0.00 & 0.03 & 0.06 \\
\hline Ni I & 0.17 & 0.01 & -0.02 & -0.02 & -0.15 & 0.08 \\
\hline $\mathrm{Cu} \mathrm{I}^{\mathrm{f}}$ & 0.11 & 0.00 & 0.01 & 0.01 & -0.01 & 0.11 \\
\hline Zn I & 0.13 & -0.05 & 0.04 & 0.00 & -0.01 & 0.11 \\
\hline Sr II & 0.13 & 0.06 & -0.01 & 0.00 & -0.04 & 0.11 \\
\hline Y II & 0.08 & 0.05 & -0.01 & 0.00 & -0.02 & 0.05 \\
\hline $\mathrm{Ba} \mathrm{II}^{\mathrm{f}}$ & 0.11 & 0.08 & -0.01 & 0.01 & -0.01 & 0.08 \\
\hline $\mathrm{La} \mathrm{II}^{\mathrm{f}}$ & 0.08 & 0.05 & 0.00 & 0.00 & 0.00 & 0.06 \\
\hline $\mathrm{Eu} \mathrm{II}^{\mathrm{f}}$ & 0.10 & 0.06 & -0.01 & 0.01 & -0.01 & 0.08 \\
\hline
\end{tabular}

Notes.

a Computed from the line list of HE1416-1032 with selected additions as required.

b $1 \sigma$ uncertainty in $[\mathrm{X} / \mathrm{Fe}]$ for a single (typical) star. Fe II is used for the ionized species. The adopted uncertainties in stellar parameters $(+100 \mathrm{~K}$ in $T_{\text {eff }},+0.25$ dex in $\log (g),+0.2 \mathrm{dex}$ in $[\mathrm{Fe} / \mathrm{H}]$ of the adopted model stellar atmosphere, and $+0.2 \mathrm{~km} \mathrm{~s}^{-1}$ in $v_{t}$ ) and from the uncertainties in the measured $W_{\lambda}$ are discussed in Section 6. Potential systematic errors are not included.

${ }^{c}$ Uncertainty in $\log \epsilon(\mathrm{Fe})$ of a single (typical) star for the adopted uncertainties in stellar parameters. Potential systematic errors are not included.

${ }^{d}$ These are of the form $[\mathrm{XI} / \mathrm{H}]-[\mathrm{XII} / \mathrm{H}]$, not [XI/Fe I] - [XII/Fe II].

e A minimum uncertainty in fitting a synthesis to an observed high resolution spectrum of either $\mathrm{CH}$ or $\mathrm{NH}$ bands corresponding to 0.05 dex is assumed.

${ }^{\mathrm{f}}$ HFS was used when necessary. See Section 6.2 for details.

g A larger uncertainty is assigned to the far UV $W_{\lambda}$.

to that observed. The ionization equilibrium for neutral versus singly ionized Fe is shown for our sample in Figure 3.

The slopes of the linear fit to deduced Fe I abundances as a function of $\chi, \log \left[W_{\lambda} / \lambda\right]$, and $\lambda$ are given in Table 6 , and, for the first two, are shown in Figure 4. The dependence on $\log \left[W_{\lambda} / \lambda\right]$ is small (i.e., the slopes are close to zero), as is also true of the dependence on $\lambda$. The quartiles for the absolute value of the slope for $\log \left[W_{\lambda} / \lambda\right]$ are only $0.017,0.028$, and $0.060 \mathrm{dex} / \mathrm{dex}$, so over the typical range of $1.8 \mathrm{dex}$ for $\log \left[W_{\lambda} / \lambda\right]$, the maximum change in deduced $\mathrm{Fe}$ abundance is only $0.03,0.05$, and $0.11 \mathrm{dex}$ respectively. Raising $v_{t}$ by $0.1 \mathrm{~km} \mathrm{~s}^{-1}$ decreases this slope by $0.05 \mathrm{dex} / \mathrm{dex}$. The spread in the lower panel of Figure 4 of this slope around zero suggests that the uncertainty in our adopted values of $v_{t}$ is about $0.1 \mathrm{~km} \mathrm{~s}^{-1}$, indicating that our choice of $v_{t}$ is appropriate.

The dependence on $\chi$ is small for the hot EMP dwarfs, but our analyses for the cooler giants tend to yield higher Fe 
Table 5

Abundance Ratio Changes for Small Changes in Stellar Parameters for Turnoff Region Stars ${ }^{\mathrm{a}}$

\begin{tabular}{|c|c|c|c|c|c|c|}
\hline Species & $\begin{array}{c}\sigma[\mathrm{X} / \mathrm{Fe}]^{\mathrm{b}} \\
1 \mathrm{Star} \\
(1 \sigma)(\mathrm{dex})\end{array}$ & $\begin{array}{c}\Delta \log \epsilon(X) \\
T_{\text {eff }} \\
(\operatorname{dex})\end{array}$ & $\begin{array}{c}\Delta \log \epsilon(X) \\
\log (g) \\
(\operatorname{dex})\end{array}$ & $\begin{array}{c}\Delta \log \epsilon(X) \\
{[\mathrm{Fe} / \mathrm{H}]} \\
(\mathrm{dex})\end{array}$ & $\begin{array}{c}\Delta \log \epsilon(X) \\
v_{t} \\
(\operatorname{dex})\end{array}$ & $\begin{array}{c}W_{\lambda} \\
(\operatorname{dex})\end{array}$ \\
\hline$[\mathrm{Fe} \mathrm{I} / \mathrm{H}]^{\mathrm{c}}$ & 0.13 & -0.08 & 0.00 & 0.04 & 0.04 & 0.01 \\
\hline$[\mathrm{Fe} \text { II } / \mathrm{H}]^{\mathrm{c}}$ & 0.09 & -0.02 & -0.07 & 0.03 & 0.03 & 0.03 \\
\hline$\left[\mathrm{Fe} \text { II } / \mathrm{Fe} \mathrm{I}_{\mathrm{I}}\right]^{\mathrm{d}}$ & 0.10 & 0.06 & -0.07 & -0.01 & -0.01 & 0.02 \\
\hline$[\mathrm{Ti} \text { II/Ti I }]^{\mathrm{d}}$ & 0.12 & 0.06 & -0.07 & -0.01 & 0.04 & -0.06 \\
\hline $\mathrm{Na} I$ & 0.06 & 0.01 & 0.00 & -0.01 & 0.02 & 0.06 \\
\hline Mg I & 0.07 & 0.02 & 0.02 & -0.03 & -0.03 & 0.05 \\
\hline $\mathrm{Al}$ I & 0.06 & 0.00 & 0.00 & 0.00 & -0.01 & 0.06 \\
\hline Si I & 0.11 & 0.01 & -0.01 & 0.02 & 0.07 & 0.08 \\
\hline $\mathrm{Ca} \mathrm{I}$ & 0.05 & 0.02 & 0.00 & 0.00 & 0.02 & 0.03 \\
\hline $\mathrm{Sc} \mathrm{II}^{\mathrm{e}}$ & 0.06 & -0.04 & 0.00 & 0.00 & 0.01 & 0.05 \\
\hline Ti I & 0.08 & 0.00 & 0.00 & -0.01 & 0.00 & 0.08 \\
\hline Ti II & 0.05 & -0.02 & 0.00 & 0.00 & 0.04 & 0.02 \\
\hline V II & 0.09 & -0.04 & -0.02 & -0.01 & 0.01 & 0.08 \\
\hline $\mathrm{Cr} \mathrm{I}$ & 0.06 & -0.01 & 0.00 & 0.00 & 0.04 & 0.04 \\
\hline $\mathrm{Mn} \mathrm{I}^{\mathrm{e}}$ & 0.07 & -0.02 & 0.00 & 0.00 & 0.04 & 0.06 \\
\hline Mn II UV & 0.14 & -0.05 & -0.03 & 0.00 & 0.10 & 0.08 \\
\hline $\operatorname{Co~I}^{\mathrm{e}}$ & 0.05 & -0.02 & 0.00 & 0.00 & 0.01 & 0.05 \\
\hline $\mathrm{Ni} \mathrm{I}$ & 0.08 & -0.02 & 0.00 & 0.00 & 0.06 & 0.06 \\
\hline $\mathrm{Cu}$ I UVe,f & 0.12 & -0.04 & 0.01 & -0.01 & 0.01 & 0.11 \\
\hline Zn I & 0.07 & 0.02 & -0.02 & -0.01 & 0.01 & 0.06 \\
\hline Sr II & 0.11 & -0.04 & 0.00 & 0.00 & 0.08 & 0.06 \\
\hline $\mathrm{Ba} \mathrm{II}^{\mathrm{e}}$ & 0.08 & -0.06 & -0.01 & 0.00 & 0.01 & 0.06 \\
\hline $\mathrm{Eu} \mathrm{II}$ & 0.12 & -0.06 & -0.06 & 0.02 & 0.00 & 0.08 \\
\hline
\end{tabular}

Notes.

${ }^{a}$ Computed from the line list of HE2344-2800, except for Eu II, which is from HE0458-1346.

b $1 \sigma$ uncertainty in $[\mathrm{X} / \mathrm{Fe}]$ for a single (typical) star. Fe II is used for the ionized species. The adopted uncertainties in stellar parameters $\left(+100 \mathrm{~K}\right.$ in $T_{\text {eff }}$, +0.2 dex in $\log (g),+0.2$ dex in $[\mathrm{Fe} / \mathrm{H}]$ of the adopted model stellar atmosphere, $+0.2 \mathrm{~km} \mathrm{~s}^{-1}$ in $v_{t}$, and from the uncertainties in the measured $W_{\lambda}$ are discussed in Section 6. Potential systematic errors are not included.

${ }^{c}$ Uncertainty in $\log \epsilon(\mathrm{Fe})$ of a single (typical) star for the adopted uncertainties in stellar parameters. Potential systematic errors are not included.

$\mathrm{d}$ These are of the form $[\mathrm{XI} / \mathrm{H}]-[\mathrm{XII} / \mathrm{H}]$, not $[\mathrm{XI} / \mathrm{Fe} \mathrm{I}]-[\mathrm{XII} / \mathrm{Fe} \mathrm{II}]$.

e HFS was used when necessary. See Section 6.2 for details.

${ }^{\mathrm{f}} \mathrm{A}$ larger uncertainty is assigned to the far $\mathrm{UV} W_{\lambda}$.

Table 6

Slopes of Linear Fits to Abundances of Individual Fe I Lines

\begin{tabular}{lcccc}
\hline \hline ID & \multirow{2}{*}{ N Lines } & \multicolumn{3}{c}{ Slopes } \\
\cline { 3 - 5 } & & $\mathrm{EP}^{\mathrm{a}}$ & $\begin{array}{c}W_{\lambda} / \lambda^{\mathrm{b}} \\
(\mathrm{dex})\end{array}$ & $\begin{array}{c}\lambda^{\mathrm{c}} \\
\left(\mathrm{dex} \AA^{-1}\right)\end{array}$ \\
\hline HE0007-1832 & 18 & $\ldots$ & $\ldots$ & $\ldots$ \\
HE0009-6039 & 31 & -0.017 & -0.043 & $0.4672 \mathrm{E}-04$ \\
HE0011-0035 & 63 & -0.092 & -0.021 & $0.1104 \mathrm{E}-04$ \\
HE0012-1441 & 14 & $\ldots$ & $\ldots$ & $\ldots$ \\
HE0017-4346 & 15 & $\ldots$ & $\ldots$ & $\ldots$ \\
\hline
\end{tabular}

Notes.

a This is $\Delta[\log (\epsilon(\mathrm{Fe})] / \Delta(\chi)$, where $\chi$ is the excitation potential of the lower level of the line.

b This is $\Delta\left[\log (\epsilon(\mathrm{Fe})] / \Delta\left(W_{\lambda} / \lambda\right)\right.$.

${ }^{\mathrm{c}}$ This is $\Delta[\log (\epsilon(\mathrm{Fe})] / \Delta(\lambda)$, where $\lambda$ is the wavelength of the transition.

(This table is available in its entirety in a machine-readable form in the online journal. A portion is shown here for guidance regarding its form and content.)

abundances from the $\mathrm{Fe} \mathrm{I}$ lines with the lowest $\chi$. A decrease in $T_{\text {eff }}$ of $\sim 300 \mathrm{~K}$ would be required to make the mean slope of the linear fit to deduced $\mathrm{Fe}$ I abundances as a function of $\chi$ as shown in Figure 4 become zero, but this introduces problems

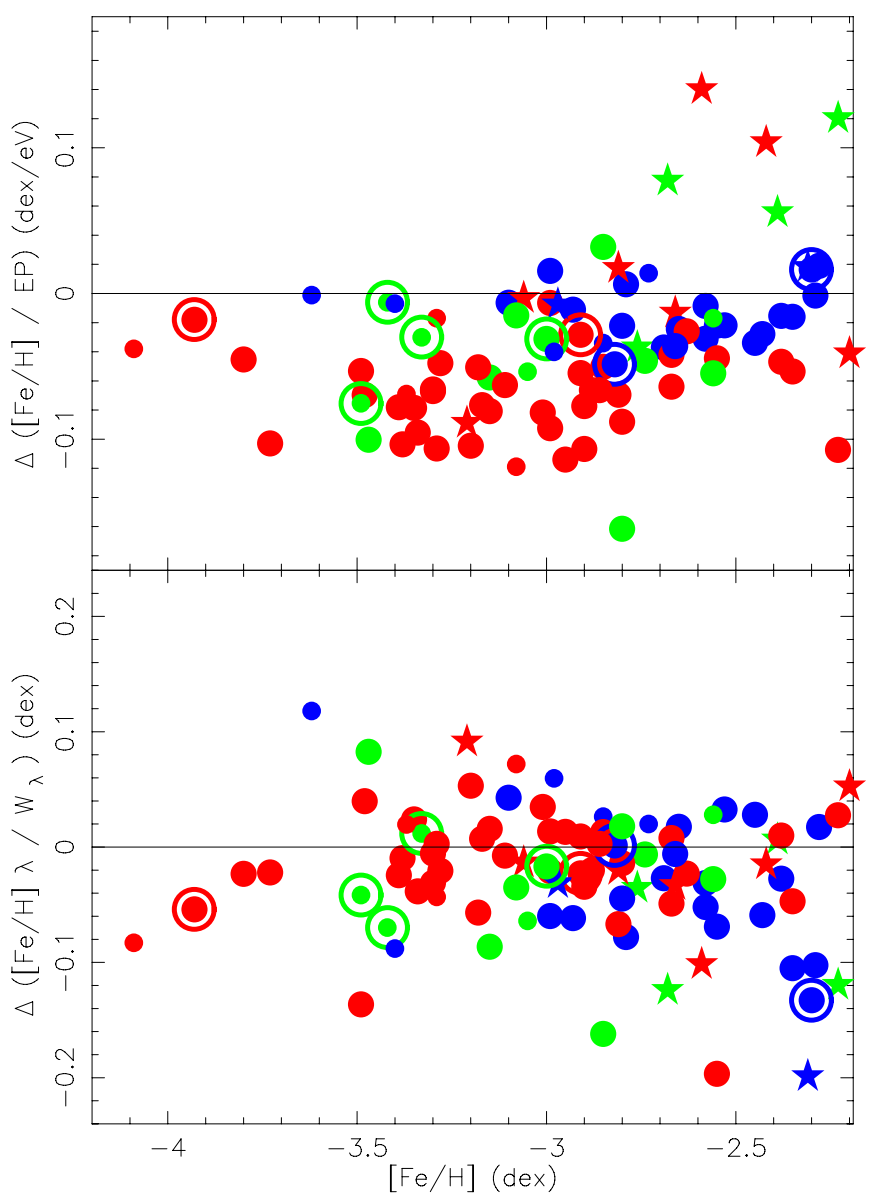

Figure 4. Slopes of deduced Fe abundance from Fe I lines as a function of $\chi$ (typical range: 0 to $4.5 \mathrm{eV}$; upper panel) or for $\log \left(W_{\lambda} / \lambda\right)$ (typical range: -5.8 to -4.6 ; lower panel) are shown for EMP stars with more than 20 detected Fe I lines. Symbols are as in Figure 3. Smaller symbols are used for stars with less than 40 detected $\mathrm{Fe}$ I lines.

(A color version of this figure is available in the online journal.)

in the ionization equilibrium. No matter how we tried to adjust the various stellar parameters, the coolest giants tend to have somewhat negative slopes for the deduced $\mathrm{Fe}$ abundance from $\mathrm{Fe} \mathrm{I}$ lines as a function of $\chi$; the $0 \mathrm{eV} \mathrm{Fe} \mathrm{I}$ lines almost always tend to be too strong in such cool giants. Use of MOOG2010-SCAT instead of MOOG-2002 does not eliminate this problem. Presumably this arises from some deficiency in the model stellar atmospheres we adopt or in the analysis. Perhaps this is a symptom of non-LTE, as suggested by the calculations of Bergemann et al. (2012). In any case, since most of the Fe I lines we use have $\chi>2 \mathrm{eV}$, the effect of this problem is not large. However, it must be recognized that for cool giants, something is systematically not correct, while for the hotter EMP dwarfs, the analyses are completely satisfactory. Since Fe II is the dominant species, many authors, including for example Thevenin \& Idiart (1999) and Bergemann et al. (2012), recommend using the abundance derived from $\mathrm{Fe}$ II lines rather than that from the more numerous and stronger $\mathrm{Fe} \mathrm{I}$ lines as the $\mathrm{Fe}$ abundance. Unfortunately with such faint EMP stars, the detectable Fe II lines are often very few in number and very weak.

The final derived abundance ratios for our sample of 122 EMP stars, primarily from the HES, are shown as a function of $[\mathrm{Fe} / \mathrm{H}]$ in Figures 5-9. Each of these figures shows the abundance ratios for four species, including, with the exception of Eu, upper limits. We set the vertical scale to be identical on as 


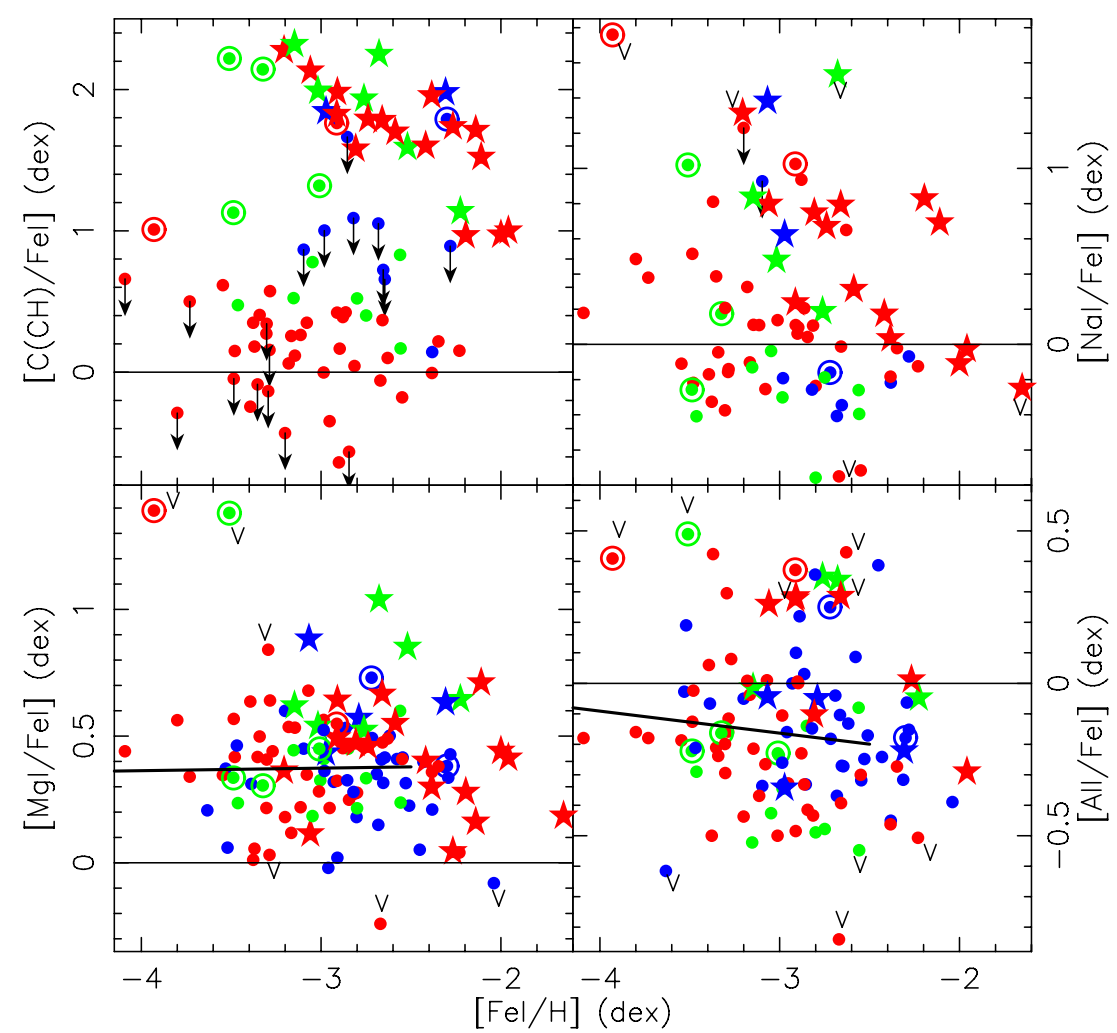

Figure 5. $[\mathrm{C} / \mathrm{Fe}]$ (upper left), $[\mathrm{Na} / \mathrm{Fe}]$ (upper right), $[\mathrm{Mg} / \mathrm{Fe}]$ (lower left) and $[\mathrm{Al} / \mathrm{Fe}]$ vs. $[\mathrm{Fe} / \mathrm{H}]$ for our 0Z sample. Symbols are as in Figure 3. "V" denotes an abundance ratio that was carefully checked and "?" denotes one which when checked could not be verified. The linear fits from Table 13 are shown when available. Three $\mathrm{C}$-rich stars from our sample have $[\mathrm{C} / \mathrm{Fe}]$ beyond the maximum range shown in the upper left panel.

(A color version of this figure is available in the online journal.)

many panels in a given one of this set of five figures as possible, but this was not always feasible.

\subsection{Non-LTE}

The only correction for non-LTE uniformly implemented here in the tables and figures is +0.6 dex for $\mathrm{Al}$ for all stars based on the calculations of Baumüller \& Gehren (1997). Only the resonance doublet at $3950 \AA$ can be detected in these EMP stars; all other Al I lines are too weak. Often only the $3961 \AA$ line can be used as the $3944 \AA$ line will be blended with strong CH lines if $\mathrm{C}$ is enhanced.

Extensive calculations of non-LTE effects in EMP stars have recently become available for other species. Non-LTE corrections for $\mathrm{Ca}$ lines have been calculated by several groups, most recently by Spite et al. (2012). At the relevant low metallicities, they are negligible in EMP giants for the resonance line of Ca I at $4226 \AA$, but can reach +0.3 dex in EMP giants (they are close to zero for dwarfs) for typical subordinate lines of this species. The $\mathrm{Ca}$ II line at $3736 \AA$ with $\chi=3.1 \mathrm{eV}$ was picked up in a small number of stars. It presumably should be relatively unaffected by non-LTE, but is a blend in the blue wing of a much stronger $\mathrm{Fe}$ I line, hence difficult to measure accurately. We cannot comment on potential differences between abundances inferred from these two ionization states of $\mathrm{Ca}$ with this specific $\mathrm{Ca}$ II line from our work.

Other recent calculations include Andrievsky et al. (2010) for $\mathrm{Mg}$, Lind et al. (2011) for $\mathrm{Na}$, Andrievsky et al. (2011) for Sr, and Dobrovolskas et al. (2012) for Ba. For Mg, the non-LTE corrections are about +0.1 dex for EMP giants and +0.3 dex for EMP turnoff stars. Andrievsky et al. (2011) find that for the resonance lines of $\mathrm{Sr}$ II at 4077 and $4215 \AA$, the only ones used here, the non-LTE corrections are approximately constant at +0.4 dex for EMP dwarfs and are smaller and negative for EMP giants. However, Hansen et al. (2013) find much smaller corrections for stars at $[\mathrm{Fe} / \mathrm{H}] \sim-3.0$ of $\mathrm{Sr}$ abundance from the $4077 \AA \mathrm{Sr}$ II line of +0.10 dex and -0.05 dex for dwarfs and giants, respectively. The non-LTE corrections for Ba II are about -0.3 dex for hot turnoff EMP stars, becoming larger for subgiants, and reaching +0.3 dex for the coolest EMP red giants. However, as we will see later, the range in $\mathrm{Sr}$ and $\mathrm{Ba}$ abundance is so large that the differences in non-LTE corrections with $T_{\text {eff }}$ for EMP stars are to first order irrelevant.

Non-LTE corrections for the $7699 \AA$ line of K I (the only one detected here) have been calculated by several groups (e.g., Ivanova \& Shimanskiı̌ 2000; Takeda et al. 2002; Andrievsky et al. 2010). These non-LTE corrections are negative, and range from -0.1 to -0.9 dex (see Figure 6 of Ivanova \& Shimanskiı 2000). They vary strongly with $T_{\text {eff }}$ and with metallicity, but they have not been applied to our deduced abundances.

There is also the issue of corrections for three-dimensional (3D) effects, which tend to affect lines primarily formed in the outer layers of RGB giants; see, e.g., Asplund (2005). This becomes important in comparing abundances for a specific element deduced from molecules versus those from high excitation atomic lines, such as occurs with $\mathrm{O}$, where the very limited available features include $\mathrm{OH}$, very high excitation $\mathrm{O}$ I lines (the triplet near $7770 \AA$ ), and the extremely weak forbidden lines, impossible to detect in these EMP stars. Pending the availability of large grids of models that include such effects, which is still computationally demanding, we ignore 3D effects, but we note that for some atomic lines, and in particular for low excitation Fe I lines in metal-poor stars, they sometimes work in the 


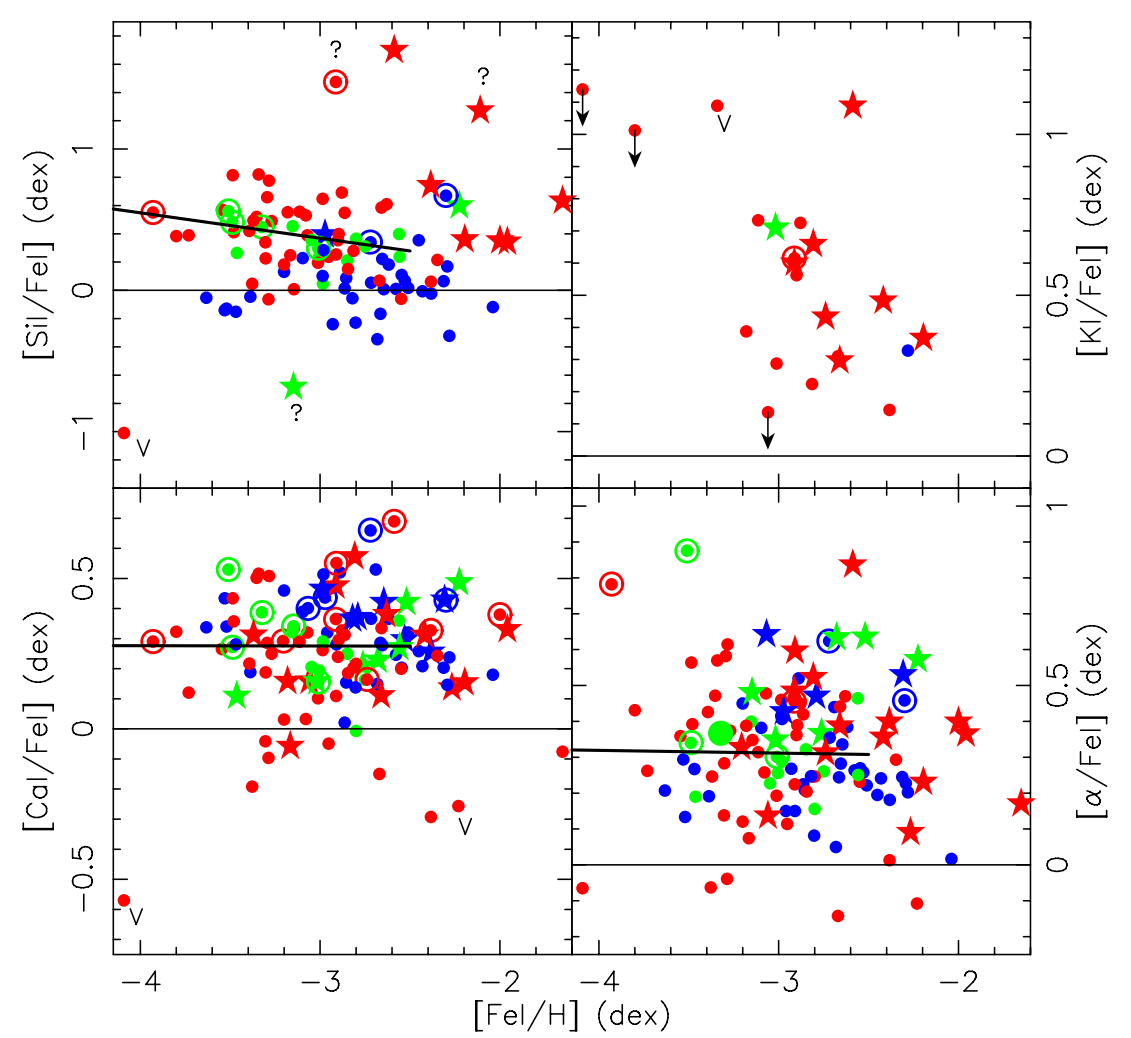

Figure 6. $[\mathrm{Si} / \mathrm{Fe}]$, with the linear fit for giants, (upper right), $[\mathrm{K} / \mathrm{Fe}]$ (upper left), $[\mathrm{Ca} / \mathrm{Fe}]$ (lower left), and $[\alpha / \mathrm{Fe}]$ (lower right) vs. [Fe/H] for the $0 \mathrm{Z}$ sample. Symbols are as in Figure 3. Notes regarding checks of outliers are as in Figure 5. The linear fits from Table 13 are shown when available.

(A color version of this figure is available in the online journal.)

opposite direction so as to cancel at least partially the effects of non-LTE; see, e.g., Section 5.3 of Magic et al. (2013).

Since our primary interest here is outliers from well characterized abundance ratio trends, these limitations in our abundance analyses should not be a serious impediment.

\subsection{Other Analysis Issues}

The uncertainties in the deduced abundance ratios are given in Table 4 (for giants) and Table 5 (for dwarfs). These include terms from the adopted uncertainty in $T_{\text {eff }}$ of $100 \mathrm{~K}$, that of $\log (g)$ of 0.25 dex for giants (a slightly smaller value is adopted for main sequence turnoff stars), that from a change in $[\mathrm{Fe} / \mathrm{H}]$ of $+0.2 \mathrm{dex}$ for the model stellar atmosphere used, and that from a change in $v_{t}$ of $0.2 \mathrm{~km} \mathrm{~s}^{-1}$. A term for the uncertainty in the measurements of $W_{\lambda}$ is also included, set to $0.11 / \sqrt{N \text { (lines) }}$ dex for the giants, where $N$ (lines) is the number of detected absorption lines of the species. A somewhat lower value for a single detected line, 0.08 dex, was used for the dwarfs, where in general due to their higher $T_{\text {eff }}$, the lines are weaker and less crowded. Random errors in the $g f$ values also contribute to this term. These terms are added in quadrature, with a minimum value for the total uncertainty set to 0.05 dex. Since in many cases only a few lines of a given species can be detected in these EMP stars, the total uncertainty in the abundance ratios $[\mathrm{X} / \mathrm{H}]$ is often dominated by that of the $W_{\lambda}$. Values are also given in the tables for $[\mathrm{Fe} / \mathrm{H}]$ from the neutral and ionized species and for [Fe II/Fe I].

Another issue we have looked at is whether there is any sign of differing behavior of abundance ratios between the cool giants and the hotter turnoff region stars. While in most cases no such effect can be detected, a clear separation is seen for [ $\mathrm{Si} / \mathrm{Fe}]$ as a function of $[\mathrm{Fe} / \mathrm{H}]$. For the vast majority of the stars, only the 3905 and/or $4102 \AA$ lines of Si I could be detected. As shown in Figure 6, we find that the hot dwarfs have [Si/Fe] approximately the solar value, which is consistently lower by $\sim 0.4$ dex than the values typical of the cool giants. This trend was already been noticed in our earlier work (see, e.g., Section 7.6 of Cohen et al. 2004), see also Preston et al. (2006) and, for the First Stars project, Bonifacio et al. (2009). Shi et al. (2009) presented non-LTE corrections, which they suggested might reduce the discrepancy. Subsequently Zhang et al. (2011) demonstrated that with these non-LTE corrections for the two strong Si I lines they could largely eliminate the $T_{\text {eff }}$ dependence, and that the hot turnoff stars have positive non-LTE corrections of $\sim+0.25$ dex, making the ratio $[\mathrm{Si} / \mathrm{Fe}]$ somewhat above the solar value in EMP stars.

A smaller such trend appears to be present for the ratio $[\mathrm{Co} / \mathrm{Fe}]$ versus $[\mathrm{Fe} / \mathrm{H}]$, with the dwarfs having this abundance ratio consistently higher by $\sim 0.2 \mathrm{dex}$ (see Figure 7 ). Still smaller trends of separation in abundance ratios at a given $[\mathrm{Fe} / \mathrm{H}]$ between dwarfs and giants, to be discussed later, appear to be present.

Since some of our HIRES spectra extend far into the UV at reasonable $\mathrm{S} / \mathrm{N}$, another issue of interest is to compare the abundances deduced from features in the UV versus in the commonly utilized optical features. Fe II has three strong lines near $3260 \AA$ which are stronger than those at optical wavelengths. We find that for the eight $\mathrm{C}$-normal stars in which we could determine reliable $W_{\lambda}$ for at least two of the three lines, the mean difference in deduced $\mathrm{Fe}$ abundance is only 0.09 dex, with $\sigma$ about the mean of only 0.13 dex. This is very good agreement for such a difficult measurement.

The Mn II triplet near $3470 \AA$ was picked up in 23 C-normal stars in our sample. These UV lines with $\chi \sim 1.8 \mathrm{eV}$ give a Mn 


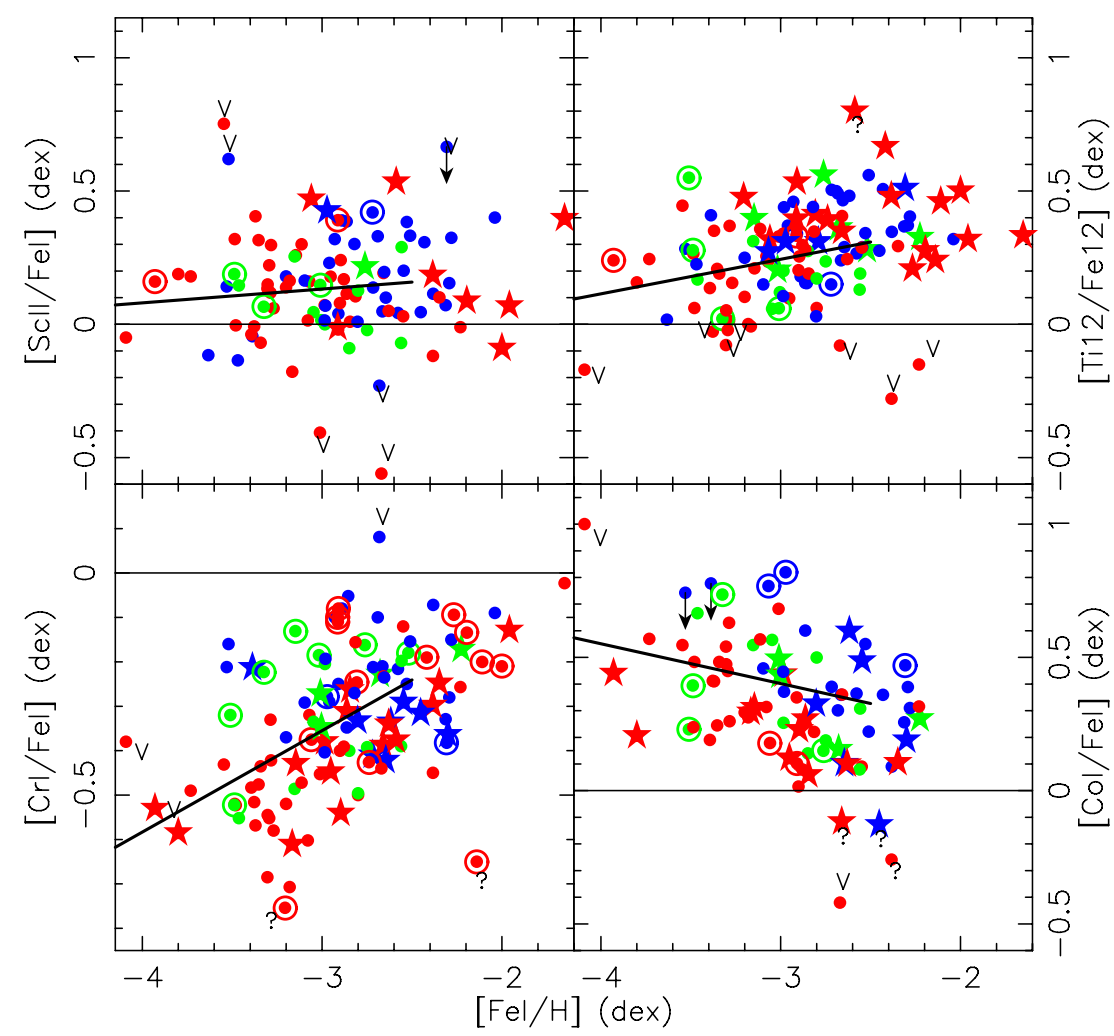

Figure 7. [Sc II/Fe] (upper left), [Ti12/Fe12], which is the mean of [Ti I/Fe I] and [Ti II/Fe II] (upper right), [Cr/Fe] (lower left), and [Co/Fe] (lower right) vs. [Fe/H] for the $0 \mathrm{Z}$ sample. Symbols are as in Figure 3. Notes regarding checks of outliers are as in Figure 5. The linear fits from Table 13 are shown when available.

(A color version of this figure is available in the online journal.)

abundance $\sim 0.3$ dex (with $\sigma=0.14$ dex) higher than the 4030 , $4033 \AA$ blue lines, which are the strongest optical lines, and the only ones usually detected in most EMP stars. (The third line of this Mn I triplet at $4034.5 \AA$ is badly blended and was not used.) Ignoring two major outliers and one star with a rather noisy spectrum at $3470 \AA$ (HE2339-5105) for which only one of the three Mn II UV lines has a measured $W_{\lambda}$, a fit to the 20 remaining $\mathrm{C}$-normal stars finds $[\mathrm{Mn}$ II/Fe II] at $[\mathrm{Fe} / \mathrm{H}] \sim$ -3.3 dex is -0.46 dex, with a small, negative, and uncertain slope. This value is 0.30 dex higher than that for the neutral species as determined from the 4030, $4033 \AA$ resonance lines. Since the bulk of $\mathrm{Mn}$ atoms are singly ionized at the relevant temperatures, we therefore infer that the Mn II abundances are probably correct, and that there are problems (presumably nonLTE effects, as suggested by a non-LTE calculation for Mn by Bergemann \& Gehren 2008) with the analysis for the resonance Mn I $4030 \AA$ lines.

Cayrel et al. (2004) suggested that Mn abundances derived from the $4030 \AA \mathrm{Mn}$ I resonance triplet require a correction of $\sim+0.3$ dex compared to those deduced from the redder and much weaker subordinate Mn I lines. In principle, our spectra could be used to check this. However, the subordinate optical Mn I lines are so weak that we cannot ensure that their measured $W_{\lambda}$ are sufficiently accurate. This test is best done with a sample of somewhat higher $[\mathrm{Fe} / \mathrm{H}]$ and with brighter stars than that presented here. If this value for the offset holds, the $[\mathrm{Mn} / \mathrm{Fe}]$ ratio inferred from the $4030 \AA$ resonance lines of Mn I would then be quite close (within $0.1 \mathrm{dex}$ ) to that from the Mn II UV lines.

Table 7 presents the list of lines we have adopted with their atomic parameters, and the number of stars in which each line was detected. Hyperfine structure (HFS) corrections were
Table 7

Atomic Data for Lines Used

\begin{tabular}{lcccc}
\hline $\begin{array}{l}\lambda \\
(\AA)\end{array}$ & Elem $^{\mathrm{a}}$ & $\begin{array}{c}\chi \\
(\mathrm{eV})\end{array}$ & $\begin{array}{c}\log (g f) \\
(\mathrm{dex})\end{array}$ & $\begin{array}{c}\text { No. Stars } \\
\text { Detected }^{\mathrm{b}}\end{array}$ \\
\hline 3189.30 & $108.0^{\mathrm{c}}$ & 1.03 & -1.990 & 2 \\
3255.50 & $108.0^{\mathrm{c}}$ & 1.30 & -1.940 & 3 \\
6707.80 & 3.0 & 0.00 & 0.178 & 8 \\
6300.30 & 8.0 & 0.00 & -9.780 & 5 \\
6363.78 & 8.0 & 0.02 & -10.300 & 2 \\
\hline
\end{tabular}

Notes.

${ }^{\text {a }}$ Element code: atomic number + (ionization stage/10), i.e., $26.0=$ Fe I, $26.1=$ Fe II

${ }^{\mathrm{b}}$ Upper limits are also included.

${ }^{\mathrm{c}} \mathrm{UV} \mathrm{OH}$ lines.

(This table is available in its entirety in a machine-readable form in the online journal. A portion is shown here for guidance regarding its form and content.)

derived for Sc II, Mn I, Mn II, Co I, Cu I, Ba II, La II, and $\mathrm{Eu}$ II as necessary by comparing for each star the abundance of a species derived using a multi-component line list for each detected line with that derived from the equivalent widths of each line using the closest model atmosphere for that specific star; see Cohen et al. (2008) and references therein for the sources of the HFS patterns. The measured equivalent widths are given in Table 8; negative values denote upper limits. Table 9 gives our adopted solar abundances, which are largely from Grevesse \& Sauval (1998) with slight modifications to those of C, O, and Fe. Our derived abundances are given in Table 10. Upper limits are indicated by setting $N$ (lines) negative. As indicated in Section 1 , we have already published analyses for 74 of these 
Table 8

Equivalent Widths, First 12 Stars

\begin{tabular}{|c|c|c|c|c|c|c|c|c|c|c|c|c|c|}
\hline $\begin{array}{l}\lambda \\
(\AA)\end{array}$ & $\mathrm{HE}$ & $\begin{array}{c}\mathrm{W}_{\lambda} \\
(\mathrm{m \AA} \AA \\
0007 \\
-1832\end{array}$ & $\begin{array}{c}\mathrm{W}_{\lambda} \\
(\mathrm{m} \AA) \\
0009 \\
-6039\end{array}$ & $\begin{array}{c}\mathrm{W}_{\lambda} \\
(\mathrm{m} \AA) \\
0011 \\
-0035\end{array}$ & $\begin{array}{c}\mathrm{W}_{\lambda} \\
(\mathrm{m \AA}) \\
0012 \\
-1441\end{array}$ & $\begin{array}{c}\mathrm{W}_{\lambda} \\
(\mathrm{m} \AA) \\
0017 \\
-4346\end{array}$ & $\begin{array}{c}\mathrm{W}_{\lambda} \\
(\mathrm{m} \AA) \\
0025 \\
-2334\end{array}$ & $\begin{array}{c}\mathrm{W}_{\lambda} \\
(\mathrm{m \AA} \AA \\
0028 \\
-2152\end{array}$ & $\begin{array}{c}\mathrm{W}_{\lambda} \\
(\mathrm{m} \AA) \\
0037 \\
-0348\end{array}$ & $\begin{array}{c}\mathrm{W}_{\lambda} \\
(\mathrm{m} \AA) \\
0039 \\
-4154\end{array}$ & $\begin{array}{c}\mathrm{W}_{\lambda} \\
(\mathrm{m} \AA) \\
0048 \\
-0611\end{array}$ & $\begin{array}{c}\mathrm{W}_{\lambda} \\
(\mathrm{m} \AA) \\
0054 \\
-2123\end{array}$ & $\begin{array}{c}\mathrm{W}_{\lambda} \\
(\mathrm{m} \AA) \\
0055 \\
-2314\end{array}$ \\
\hline 6707.80 & 3.0 & $\ldots$ & -10.0 & $\ldots$ & $\ldots$ & $\ldots$ & $\ldots$ & $\ldots$ & $\ldots$ & $\ldots$ & $\ldots$ & $\ldots$ & 29.7 \\
\hline 5889.95 & 11.0 & $\ldots$ & 78.0 & $\ldots$ & $\ldots$ & 154.0 & $\ldots$ & $\ldots$ & 133.1 & 141.0 & 139.8 & & 64.9 \\
\hline 5895.92 & 11.0 & $\ldots$ & 71.0 & $\ldots$ & $\ldots$ & 125.0 & $\ldots$ & $\ldots$ & 111.0 & 122.4 & 124.2 & $\ldots$ & 51.5 \\
\hline 3829.36 & 12.0 & $\ldots$ & 101.0 & $\ldots$ & $\ldots$ & $\ldots$ & $\ldots$ & $\ldots$ & 145.2 & $\ldots$ & $\ldots$ & $\ldots$ & $\ldots$ \\
\hline 4057.52 & 12.0 & $\ldots$ & $\ldots$ & $\ldots$ & 34.0 & $\ldots$ & $\ldots$ & 9.6 & 14.5 & $\ldots$ & 29.1 & 7.2 & $\ldots$ \\
\hline
\end{tabular}

Notes.

a Element code: atomic number + (ionization stage $/ 10$ ), i.e., $26.0=\mathrm{Fe}$ I, $26.1=\mathrm{Fe}$ II.

b Negative $\mathrm{W}_{\lambda}$ denote upper limits.

(This table is available in its entirety in a machine-readable form in the online journal. A portion is shown here for guidance regarding its form and content.)

Table 9

Adopted Solar Abundances for HES Stars

\begin{tabular}{lccccc}
\hline \hline Elem $^{\mathrm{a}}$ & $\begin{array}{c}\log [\epsilon(\mathrm{X})] \\
(\mathrm{dex})\end{array}$ & Elem $^{\mathrm{a}}$ & $\begin{array}{c}\log [\epsilon(\mathrm{X})] \\
(\mathrm{dex})\end{array}$ & Elem $^{\mathrm{a}}$ & $\begin{array}{c}\log [\epsilon(\mathrm{X})] \\
(\mathrm{dex})\end{array}$ \\
\hline 6.0 & 8.59 & 7.0 & 7.93 & 8.0 & 8.93 \\
11.0 & 6.32 & 12.0 & 7.54 & 13.0 & 6.47 \\
14.0 & 7.55 & 19.0 & 5.12 & 20.0 & 6.36 \\
20.1 & 6.36 & 21.0 & 3.10 & 21.1 & 3.10 \\
22.0 & 4.99 & 22.1 & 4.99 & 23.0 & 4.00 \\
23.1 & 4.00 & 24.0 & 5.67 & 24.1 & 5.67 \\
25.0 & 5.39 & 25.1 & 5.39 & 26.0 & 7.45 \\
26.1 & 7.45 & 26.2 & 7.45 & 27.0 & 4.92 \\
28.0 & 6.25 & 29.0 & 4.21 & 30.0 & 4.60 \\
38.0 & 2.90 & 38.1 & 2.90 & 39.1 & 2.24 \\
40.1 & 2.60 & 56.1 & 2.13 & 57.1 & 1.14 \\
58.1 & 1.55 & 59.1 & 0.71 & 60.1 & 1.50 \\
62.1 & 1.00 & 63.1 & 0.51 & 64.1 & 1.12 \\
66.1 & 1.10 & 82.0 & 1.95 & 90.1 & 0.12 \\
\hline
\end{tabular}

Notes.

${ }^{a}$ Species code: atomic number + (ionization stage/10), i.e., $26.0=\mathrm{Fe}$ I, $26.1=$ Fe II. Special case: 26.2 is for three strong Fe II lines in the UV near $3260 \AA$.

b Adopted $\log [\epsilon(\mathrm{X})]$ where the value for $\mathrm{H}$ is 12.00 . stars previously; the results presented here supersede the earlier ones. Note that we assume $\log [\epsilon(\mathrm{Fe})]$ for the Sun is $7.45 \mathrm{dex}$, somewhat lower than the value adopted by many recent studies, typically 7.52 dex. Hence our derived $[\mathrm{Fe} / \mathrm{H}]$ will be higher by $\sim 0.07$ dex and our derived abundance ratios $[\mathrm{X} / \mathrm{Fe}]$ will be lower by this amount than those of many other groups.

The lowest $[\mathrm{Fe} / \mathrm{H}]$ star found in the present sample of EMP candidates from the HES is HE1424-0241, a giant at -4.1 dex. There are only four reasonably well studied stars known at significantly lower metallicity. They are HE0107-5240 (Christlieb et al. 2002) and HE1347-2326 (Frebel et al. $2005)$ at $[\mathrm{Fe} / \mathrm{H}] \leqslant-5 \mathrm{dex}, \mathrm{HE} 0557-4840$ at $-4.8 \mathrm{dex}$ (Norris et al. 2007), and the recently discovered case of SDSSJ102915+172027 at [Fe/H] -4.8 dex (Caffau et al. 2011). Each of these four UMP or HMP stars has been the subject of numerous publications, and we exclude discussion of them from the present work.

\subsection{Tailored Model Atmospheres}

As we will see later, some of the $0 \mathrm{Z}$ sample stars show peculiar abundance ratios. We picked a subset of five stars exploring the range of abnormalities we found, including the

Table 10

Relative Abundances, Stars 1-5 from the HES

\begin{tabular}{|c|c|c|c|c|c|c|c|c|c|c|c|c|c|c|c|}
\hline \multirow[t]{2}{*}{ Elem $^{\mathrm{a}}$} & \multicolumn{3}{|c|}{ HE0007-1832 } & \multicolumn{3}{|c|}{ HE0009-6039 } & \multicolumn{3}{|c|}{ HE0011-0035 } & \multicolumn{3}{|c|}{ HE0012-1441 } & \multicolumn{3}{|c|}{ HE0017-4346 } \\
\hline & $\begin{array}{c}{[\mathrm{X} / \mathrm{Fe}]^{\mathrm{b}}} \\
\quad(\mathrm{dex})\end{array}$ & $\begin{array}{c}\sigma^{\mathrm{c}} \\
(\mathrm{dex})\end{array}$ & $N^{\mathrm{d}}$ & $\begin{array}{c}{[\mathrm{X} / \mathrm{Fe}]^{\mathrm{b}}} \\
\quad(\mathrm{dex})\end{array}$ & $\begin{array}{c}\sigma^{\mathrm{c}} \\
(\operatorname{dex})\end{array}$ & $N^{\mathrm{d}}$ & $\begin{array}{c}{[\mathrm{X} / \mathrm{Fe}]^{\mathrm{b}}} \\
(\mathrm{dex})\end{array}$ & $\begin{array}{c}\sigma^{\mathrm{c}} \\
(\operatorname{dex})\end{array}$ & $N^{\mathrm{d}}$ & $\begin{array}{c}{[\mathrm{X} / \mathrm{Fe}]^{\mathrm{b}}} \\
\quad(\mathrm{dex})\end{array}$ & $\begin{array}{c}\sigma^{\mathrm{c}} \\
(\mathrm{dex})\end{array}$ & $N^{\mathrm{d}}$ & $\begin{array}{c}{[\mathrm{X} / \mathrm{Fe}]^{\mathrm{b}}} \\
\quad(\mathrm{dex})\end{array}$ & $\begin{array}{c}\sigma^{\mathrm{c}} \\
(\operatorname{dex})\end{array}$ & $N^{\mathrm{d}}$ \\
\hline 26.0 & -2.79 & 0.16 & 18 & -3.29 & 0.21 & 31 & -2.99 & 0.20 & 64 & -2.52 & 0.16 & 14 & -3.07 & 0.17 & 15 \\
\hline $6.0^{\mathrm{e}}$ & 2.66 & $\ldots$ & 1 & 0.16 & $\ldots$ & -1 & 0.00 & $\ldots$ & 1 & 1.59 & $\ldots$ & 1 & 2.90 & $\ldots$ & 1 \\
\hline 7.0 & 2.11 & $\ldots$ & 1 & $\ldots$ & $\ldots$ & $\ldots$ & $\ldots$ & $\ldots$ & $\ldots$ & 0.54 & $\ldots$ & 1 & $\ldots$ & $\ldots$ & $\ldots$ \\
\hline 11.0 & $\ldots$ & $\ldots$ & $\ldots$ & -0.16 & 0.14 & 2 & $\ldots$ & $\ldots$ & $\ldots$ & $\ldots$ & $\ldots$ & $\ldots$ & 1.38 & 0.00 & 2 \\
\hline 12.0 & 0.57 & 0.16 & 4 & 0.03 & 0.27 & 4 & 0.56 & 0.05 & 4 & 0.85 & 0.10 & 4 & 0.88 & 0.13 & 3 \\
\hline 13.0 & -0.05 & $\ldots$ & 1 & -0.16 & 0.22 & 2 & -0.11 & $\ldots$ & 1 & $\ldots$ & $\ldots$ & $\ldots$ & -0.04 & $\ldots$ & 1 \\
\hline 14.0 & $\ldots$ & $\ldots$ & $\ldots$ & -0.06 & $\ldots$ & 1 & 0.65 & $\ldots$ & 1 & $\ldots$ & $\ldots$ & $\ldots$ & $\ldots$ & $\ldots$ & $\ldots$ \\
\hline 20.0 & 0.37 & $\ldots$ & 1 & -0.10 & 0.19 & 3 & 0.26 & 0.07 & 6 & 0.42 & 0.15 & 3 & 0.40 & 0.15 & 2 \\
\hline 21.1 & $\ldots$ & $\ldots$ & $\ldots$ & 0.12 & 0.13 & 2 & 0.07 & 0.08 & 3 & $\ldots$ & $\ldots$ & $\ldots$ & 1.61 & $\ldots$ & -1 \\
\hline
\end{tabular}

Notes.

a Species code: atomic number + (ionization stage/10), i.e., $26.0=$ Fe I, $26.1=$ Fe II. Special case: 26.2 is for three strong Fe II lines in the UV near $3260 \AA$.

${ }^{\mathrm{b}}[\mathrm{X} / \mathrm{Fe}](\mathrm{Fe} \mathrm{I})$, except for Fe I, where we give $[\mathrm{Fe} / \mathrm{H}]$ from $\mathrm{Fe} \mathrm{I}$ lines only.

${ }^{c}$ rms dispersion for the lines of this species around the mean abundance ratio.

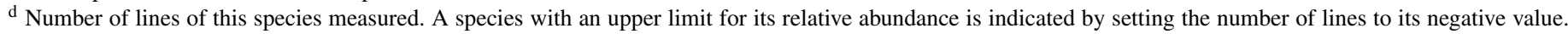

$N<-1$ means several lines were searched for, but none were firmly detected.

e $\mathrm{C}$ from synthesis of $G$ band of $\mathrm{CH}$.

(This table is available in its entirety in a machine-readable form in the online journal. A portion is shown here for guidance regarding its form and content.) 
most metal-poor C-normal star, HE1424-0241, with very low Si-abundance, HE0926-0546 (a very low metallicity giant with no $\alpha$-enhancement) and several extremely $\mathrm{C}$-enhanced stars (HE1012-1540 and HE2323-0256). For each of these stars, we computed a plane-parallel model atmosphere in LTE using ATLAS9 (Kurucz 1993) with an elemental composition constructed from the first iteration of our detailed abundance analysis. In the absence of a measured abundance for an element in a given star, the scaled solar abundance was adopted. In conformance with our more standard model atmospheres, convective overshooting was turned off in the customized models, and the mixing length parameter $(l / H)$ was 1.25 . This treatment of convection is the same as in the often-used model atmosphere grid computed by Castelli \& Kurucz (2004). We also adopted the same atomic and molecular line lists for the calculations of the opacity distribution functions as Castelli \& Kurucz (2004). The opacities were recomputed for the detailed abundance pattern of each star, then a model atmosphere tailored for that specific star was generated.

We compared the results from scaled solar composition models in the standard Kurucz grids (Kurucz 1993; our normal mode of analysis) with those when the tailored model atmospheres were used, in both cases with our adopted stellar parameters. It should be noted that our normal abundance analysis interpolates from the nearest (in $T_{\text {eff }}, \log (g)$, and $[\mathrm{Fe} / \mathrm{H}])$ models in the standard Kurucz grid, while the tailored models were computed for the adopted stellar parameters, hence no interpolation was required.

The maximum difference between $[\mathrm{Fe} / \mathrm{H}]$ as obtained from Fe I and Fe II lines at optical wavelengths was only 0.08 dex. This corresponds to the maximum change in the Fe ionization equilibrium between the two solutions, one with scaled solar abundances, and one with tailored abundances. The neutral ions show differences in $[\mathrm{X} / \mathrm{H}]$ within 0.03 dex of that for $\mathrm{Fe}$ I, while the ionized species tend to follow Fe II. The total range of the differences in $[\mathrm{X} / \mathrm{H}]$ for the absolute abundances deduced for each element considering all species with detected absorption lines between the scaled solar and the tailored model atmospheres was 0.07 dex for HE1424-0241, 0.09 dex for the very C-rich star HE2323-0256, 0.12 dex for HE1012-1540, 0.06 dex for HE0926-0546, and 0.15 dex for HE0533-5340 (among the coolest EMP giants in our sample).

The changes in abundance ratios are smaller, assuming $[\mathrm{X} / \mathrm{Fe}: \mathrm{Fe} \mathrm{I}]$ for neutral species and $[\mathrm{X} / \mathrm{Fe}: \mathrm{Fe} \mathrm{II}]$ for ionized species are used. We find from these tests that for stars with peculiar chemical inventories, an additional uncertainty in their abundance ratios of up to 0.05 dex may exist arising from this effect. Furthermore, again only for stars with severe abundance peculiarities with respect to the bulk of the large sample studied here, we may have introduced small additional errors by trying to slightly adjust the stellar parameters of the star to improve (at least as we perceive it) the results of the detailed abundance analyses.

VandenBerg et al. (2012) have recently presented a grid of stellar evolutionary tracks and isochrones for stars with enhanced abundances of one of a set of specific elements. He points out that $\mathrm{C}, \mathrm{N}$, and $\mathrm{O}$ do not contribute significantly to opacities at low temperatures and pressures, while $\mathrm{Mg}$ and $\mathrm{Si}$ do. So it is not surprising that some of the stars checked with tailored model atmospheres that are very $\mathrm{C}$ and/or $\mathrm{N}$ enhanced do not show large differences in their deduced abundances from detailed abundance analyses using our normal interpolation scheme with scaled solar composition models. VandenBerg
Table 11

Data for Candidates with $[\mathrm{Fe} / \mathrm{H}](\mathrm{HES})<-3.5 \mathrm{dex}$

\begin{tabular}{lccl}
\hline \hline ID & $\begin{array}{c}{[\mathrm{Fe} / \mathrm{H}](\mathrm{HES})} \\
(\mathrm{dex})\end{array}$ & $\begin{array}{c}{[\mathrm{Fe} / \mathrm{H}](\text { high-res })} \\
(\mathrm{dex})\end{array}$ & Comments \\
\hline HE1159-0525 & -3.52 & -2.91 & Extreme C-star \\
HE1150-0428 & -3.57 & -3.21 & C-star \\
HE2209-3909 & -3.57 & -3.47 & Hot turnoff dwarf \\
HE1031-0020 & -3.61 & -2.81 & C-star \\
HE0122-1616 & -3.63 & -2.80 & \\
HE0305-5442 & -3.68 & -3.30 & Extreme CN \\
HE0313-3640 & -3.72 & -3.63 & Hot turnoff dwarf \\
HE0911-0512 & -3.84 & $\ldots$ & M dwarf \\
HE2323-0256 & -3.96 & -3.93 & Extreme CN \\
HE1432-1819 & -4.43 & -2.56 & Hot turnoff dwarf \\
HE0208-5335 & -4.14 & $\ldots$ & QSO \\
HE1030+0137 & -4.37 & $\ldots$ & M dwarf \\
\hline
\end{tabular}

Notes.

${ }^{\text {a }} \mathrm{HE} 0305-5442$ is a rediscovery of CS22968-014. It has $[\mathrm{Fe} / \mathrm{H}]$ from Cayrel et al. (2004) of -3.56 dex.

${ }^{b} \mathrm{HE} 2323-0256$ is a rediscovery of CS22949-037. It has $[\mathrm{Fe} / \mathrm{H}]$ from Cayrel et al. (2004) of -3.79 dex.

et al. (2012) notes that it is variations for $\mathrm{Mg}$ and $\mathrm{Si}$ among the heavy elements that have the most impact on the opacity, but fortunately the bulk of the stars in our sample have about the same value of $[\mathrm{Mg} / \mathrm{Fe}]$ and of $[\mathrm{Si} / \mathrm{Fe}]$, consistent with the normal $\alpha$-enhancement seen among EMP stars. As we will see later in Section 8, only a few are highly deviant for $\mathrm{Mg}$ and/or $\mathrm{Si}$, and most of them are depleted in these elements rather than enhanced, at which point in such metal-poor stars, most of the electrons are from ionization of $\mathrm{H}$. These deviant stars were all rejected as outliers in determining the linear fits of $[\mathrm{X} / \mathrm{Fe}]$ versus $[\mathrm{Fe} / \mathrm{H}]$.

\subsection{Completeness of the High Resolution Observations}

There are 12 candidates for which $[\mathrm{Fe} / \mathrm{H}](\mathrm{HES})<-3.5 \mathrm{dex}$ from either the 2005 merge of P200 and Magellan follow up spectra or from that carried out in the spring of 2007. All of these have been observed with MIKE or HIRES. A comparison of $[\mathrm{Fe} / \mathrm{H}](\mathrm{HES})$ versus that obtained from a detailed abundance analysis is given in Table 11. Two of these twelve turned out to be M dwarfs with strong emission in the core of the $3933 \AA$ line and one is a QSO.

22 of the 28 EMP candidate stars with $-3.2<[\mathrm{Fe} / \mathrm{H}](\mathrm{HES})$ $<-3.5$ dex from the 2007 June merge of the Magellan and Palomar mod-res spectra using $[\mathrm{Fe} / \mathrm{H}](\mathrm{HES})$ derived at that time have been observed at high spectral resolution (see Table 12). Four of the six without a high resolution spectrum are extremely C-enhanced; these four probably have true values of $[\mathrm{Fe} / \mathrm{H}]$ higher than -3.2 dex.

A perusal of Tables 11 and 12 demonstrates the prevalence of the underestimation of $[\mathrm{Fe} / \mathrm{H}](\mathrm{HES})$ in extremely C-rich stars discussed in Cohen et al. (2005). It is also interesting to note that some of the higher $[\mathrm{Fe} / \mathrm{H}]$ interlopers in these two groups have abnormally low $[\mathrm{Ca} / \mathrm{Fe}]$ ratios, which is probably why their $[\mathrm{Fe} / \mathrm{H}](\mathrm{HES})$ values are significantly lower than their $[\mathrm{Fe} / \mathrm{H}](\mathrm{HIRES})$ values.

A large number of stars with $-3.2<[\mathrm{Fe} / \mathrm{H}](\mathrm{HES})<$ -2.8 dex from the 2007 June P200+Magellan merge have also been observed with either MIKE or HIRES. 
Table 12

Data for Candidates with $-3.4<[\mathrm{Fe} / \mathrm{H}](\mathrm{HES})<-3.2$ dex

\begin{tabular}{|c|c|c|c|}
\hline ID & $\begin{array}{c}{[\mathrm{Fe} / \mathrm{H}](\mathrm{HES})} \\
(\mathrm{dex})\end{array}$ & $\begin{array}{c}{[\mathrm{Fe} / \mathrm{H}] \text { (high-res) }} \\
(\mathrm{dex})\end{array}$ & Comments \\
\hline HE0014-3607 & -3.21 & $\ldots$ & Prob. extreme C-star \\
\hline HE2356-0410 & -3.22 & -3.06 & Extreme C-star \\
\hline HE1509-0806 & -3.22 & -2.91 & Extreme C-star \\
\hline HE0218-4136 & -3.22 & $\ldots$ & \\
\hline HE2203-3723 & -3.22 & -3.30 & \\
\hline HE2128-4248 & -3.23 & $\ldots$ & \\
\hline HE $1500-1628$ & -3.25 & -2.38 & v. low $[\mathrm{Ca} / \mathrm{Fe}]$ \\
\hline HE2155-3750 & -3.28 & -2.59 & Extreme C-star \\
\hline HE0349-0045 & -3.28 & -2.23 & v. low $[\mathrm{Ca} / \mathrm{Fe}]$ \\
\hline HE0048-0611 & -3.28 & -2.67 & \\
\hline HE2129-4652 & -3.28 & $\ldots$ & Prob. extreme C-star \\
\hline HE2122-4707 & -3.29 & -2.42 & C-star \\
\hline HE0024-2523 & -3.32 & -2.72 & C-star \\
\hline HE2339-5105 & -3.32 & -2.88 & \\
\hline HE0216-3838 & -3.33 & $\ldots$ & Prob. extreme C-star \\
\hline HE2251-0821 & -3.34 & -2.91 & Very strong $\mathrm{CH}$, no $\mathrm{C}_{2}$ \\
\hline HE0926-0546 & -3.35 & -3.73 & \\
\hline HE2357-0701 & -3.35 & -3.17 & \\
\hline HE0009-6039 & -3.35 & -3.29 & \\
\hline HE0344-0243 & -3.36 & -3.35 & \\
\hline HE0226+0103 & -3.36 & -2.81 & \\
\hline HE2215-2548 & -3.36 & -3.01 & \\
\hline HE1434-1442 & -3.42 & -2.39 & C-star \\
\hline HE1245-0215 & -3.42 & -2.85 & Hot turnoff star \\
\hline HE1416-1032 & -3.44 & -3.20 & \\
\hline HE0134-2504 & -3.45 & -2.74 & C-star \\
\hline HE0212-0557 & -3.45 & -2.27 & Extreme C-star \\
\hline HE2138-4250 & -3.46 & $\ldots$ & Prob. extreme C-star \\
\hline
\end{tabular}

\section{COMPARISON WITH PREVIOUS ABUNDANCE ANALYSES}

The abundance analyses we present here for EMP candidates from the HES were, as indicated above, carried out using the same method of determination of the stellar parameters, master line list, transition probabilities as well as other atomic parameters, and abundance analysis code for all the stars with the exception of nine of the stars from the Keck Pilot Project for which subsequent repeat observations were not obtained. Here we compare our results from detailed abundance analyses to the preliminary $[\mathrm{Fe} / \mathrm{H}]$ determined from our earlier moderate resolution followup spectra for each candidate and to detailed abundance analyses carried out by other major groups for the small number of stars previously analyzed. The goal from these comparisons is to establish that the work of the $0 \mathrm{Z}$ project is sufficiently robust that we are able to determine the trends among abundance ratios, and that stars we call outliers really are outliers from these mean trends. Note again that we assume $\log [\epsilon(\mathrm{Fe})]$ for the Sun is 7.45 dex, somewhat lower than the value adopted by many recent studies, typically 7.52 dex. Hence our derived $[\mathrm{Fe} / \mathrm{H}]$ will be higher by $\sim 0.07$ dex while our abundance ratios $[\mathrm{X} / \mathrm{Fe}]$ will be $\sim 0.07$ dex lower than those of many other groups.

\subsection{Comparison of the Fe-Metallicities from the HES versus High Resolution Spectra}

We compare the Fe metallicities derived from our moderate dispersion follow up spectra using a simple algorithm based on the strength of the $3933 \AA \mathrm{Ca}$ II line plus $\mathrm{H} \delta$ with those from our high resolution spectra and a detailed abundance analysis. The former was the initial basis for similar algorithms used by many recent large surveys of stellar spectra; see, e.g., Lee et al. (2008) describing the pipeline developed for the SEGUE SDSS-II. Such a comparison is therefore quite illuminating. One must, however, remember that these codes have been extensively improved over the past decade and their current versions are much more sophisticated, yielding better performance than the early versions available to us in the initial phases of this project.

A comparison of the metallicities from the 2007 HES algorithm with those found from our entire sample of high resolution spectroscopy (122 stars), ignoring the extreme C-stars for which the algorithm fails (Cohen et al. 2005) and the 2007 version does not return a value, shows that for the 35 dwarfs near the main sequence turnoff (i.e., stars with $T_{\text {eff }}>6000 \mathrm{~K}$ ), the HES algorithm underestimates the metallicity by $0.27 \mathrm{dex}$ in the mean (with $\sigma=0.36 \mathrm{dex}$ ). The EMP main sequence turnoff region dwarfs have very weak lines, so it is perhaps not surprising that moderate resolution spectra underestimate their metal abundances. Presumably recent updates in the calibration of the metallicity algorithm improve this situation. For the 87 cooler subgiants and giants, we find the mean difference is small ( $0.07 \mathrm{dex})$, but the dispersion is even larger, 0.44 dex. These differences for the full sample are shown in Figure 10.

\subsection{Comparison with the First Stars Project}

We have compared our results for stars in common with a number of other major projects focusing on EMP Galactic halo field stars, in particular the First Stars large project at the VLT (Cayrel et al. 2004), in some of our earlier publications; see, e.g., Section 7 and Appendix B of Cohen et al. (2008). First we note that the sample for their project, by design, is deliberately strongly biased against $\mathrm{C}$-rich stars. There we found that there is a $\sim 0.2$ dex offset in the absolute abundance scale between our $0 \mathrm{Z}$ project and the First Stars project. Some of the factors giving rise to this are discussed in detail in Cohen et al. (2004). We have now found one more term contributing to this systematic difference in derived $[\mathrm{Fe} / \mathrm{H}]$. With the use of the 2010 version of MOOG with a better treatment of isotropic scattering (Sobeck et al. 2011), we find that for the most metal-poor stars in our sample, $[\mathrm{Fe} / \mathrm{H}]$ is decreased by 0.1 to 0.15 dex as compared to the 2002 version of MOOG (Sneden 1973). The abundance analysis code used by the First Stars project already contained a suitable treatment of scattering in 2004. This improvement, together with the factors discussed in our earlier papers, helps to explain the difference in $[\mathrm{Fe} / \mathrm{H}]$ between the First Stars results and our earlier results for these two stars.

There are only two stars in common. HE2323-0256 (aka CS 22949-037) was discovered by McWilliam et al. (1995b). This star was subsequently reobserved at higher $\mathrm{S} / \mathrm{N}$ and analyzed by Depagne et al. (2002) and also by Cayrel et al. (2004). Comparing our current analysis of the spectrum of HE2323-0256 with that of Cayrel et al. (2004), we first note that our stellar parameter assignment is essentially identical as that of the First Stars project, $4900 \pm 125 \mathrm{~K}$ for $T_{\text {eff }}$ versus $4915 \pm 15 \mathrm{~K}$, $\log (g) 1.5$ versus $1.7 \mathrm{dex}$, and $v_{t} 1.8$ versus $1.9 \mathrm{~km} \mathrm{~s}^{-1}$ (First Stars versus $0 \mathrm{Z}$ ). Our $0 \mathrm{Z}$ analysis, which now uses the updated version of MOOG (Sobeck et al. 2011) with a tailored model atmosphere, yields $\log (\epsilon)$ for $\mathrm{Fe}$ I (Fe II) of 3.52 (3.59) dex, while that of the First Stars project is 3.51 (3.56) dex, which is remarkably good agreement between the two completely independent observations and analyses. The abundance ratios $[\mathrm{X} / \mathrm{Fe}]$ for the 15 species in common also agree well, with 10 of these having differences $|\Delta| \leqslant 0.15$ dex. The agreement for the second star in common, BS 16467-062, is not as good. Our $T_{\text {eff }}$ 


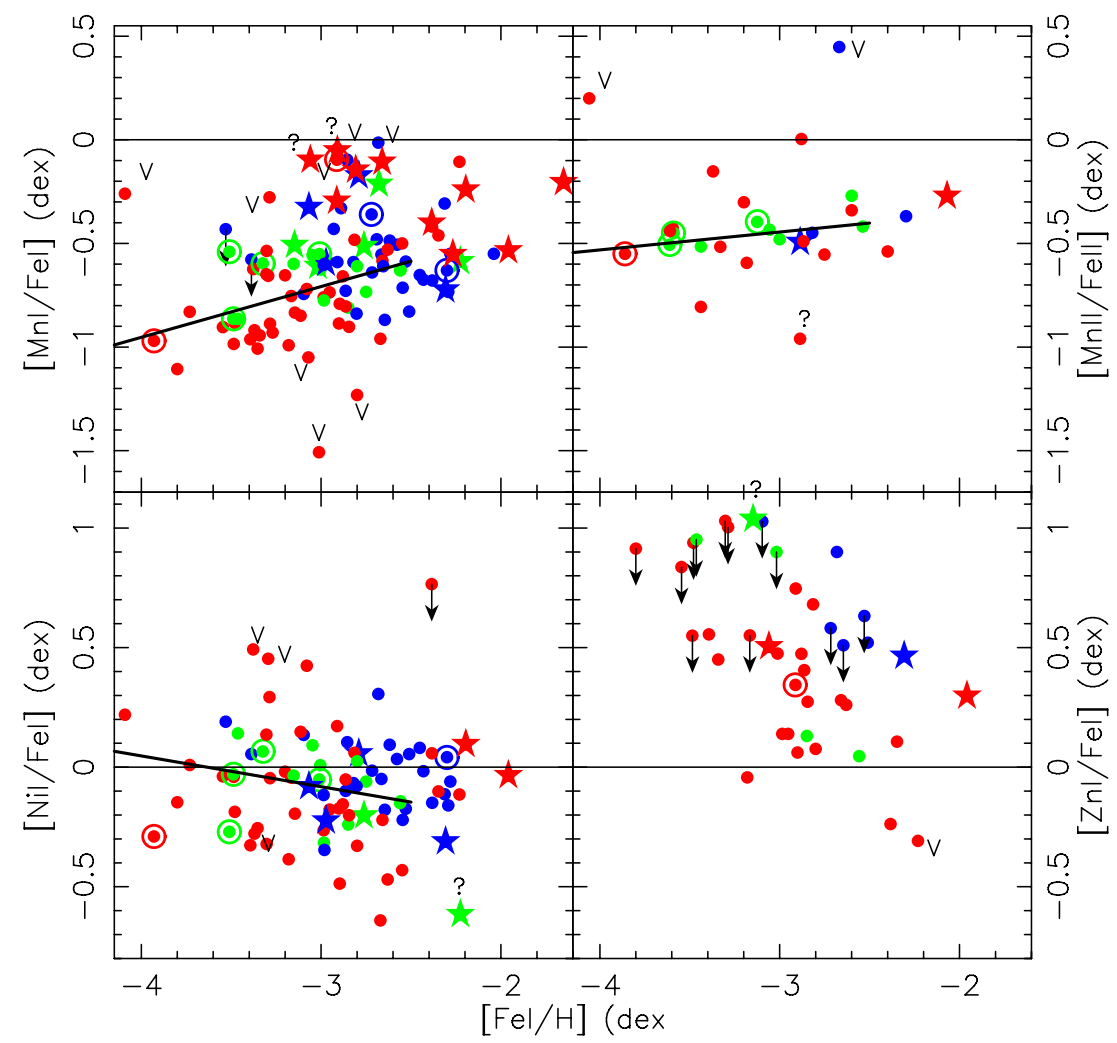

Figure 8. [Mn II/Fe] (upper right), [Mn I/Fe] (upper left), [Ni/Fe] (lower left), and [Zn/Fe] (lower right) vs. [Fe/H] for the 0Z sample. Symbols are as in Figure 3. Notes regarding checks of outliers are as in Figure 5. The linear fits from Table 13 are shown when available.

(A color version of this figure is available in the online journal.)

is $164 \mathrm{~K}$ higher than that of the First Stars project (Cayrel et al. 2004), in part because of the difference in the adopted interstellar absorption along the line-of-sight to this star. This difference in stellar parameters leads to a 0.3 dex difference in $[\mathrm{Fe} / \mathrm{H}]$, with our value being higher. If we had adopted the $T_{\text {eff }}$ used by the First Stars project, the disagreement in $[\mathrm{Fe} / \mathrm{H}]$ would become much smaller. Comparing derived abundance ratios $[\mathrm{X} / \mathrm{Fe}]$, the agreement even for BS 16467-062 is fairly good; 9 of the 13 species in common have $|\Delta| \leqslant 0.15$ dex.

\subsection{Comparison with Other Groups}

HE1300+0157 was the only star found by the HERES project (Barklem et al. 2005) to have $[\mathrm{Fe} / \mathrm{H}]<-3.5$ dex. We observed this star with HIRES in 2006 April. A detailed abundance analysis based on high S/N HDS/Subaru spectra was carried out by Frebel et al. (2007). Our adopted $T_{\text {eff }}$ is $100 \mathrm{~K}$ hotter than that of Frebel et al. (2007; a difference no larger than the uncertainties), and thus our $[\mathrm{Fe} / \mathrm{H}]$ is higher as well. The agreement between our results and those of Frebel et al. (2007) for the abundance ratios $[\mathrm{X} / \mathrm{Fe}]$ for this star is excellent, with the difference in abundance ratios exceeding 0.12 dex for only three of the 15 species in common.

Johnson \& Bolte (2002) published abundances for the rare earths and a few other elements in the carbon star HE0058-0244 (aka CS22183-015). The $T_{\text {eff }}$ are very different, that of the $0 Z$ project is $420 \mathrm{~K}$ higher than that of Johnson \& Bolte (2002). Given that they focused on the rare earths, each of which only has a few relatively weak lines, and that this is a rather crowded spectrum since the star is a carbon star, the agreement between the two independent analyses for the derived abundance ratios is reasonable.
There are two stars in common with the recent study of Norris and collaborators (Norris et al. 2013a; Yong et al. 2013), HE1346-0427 and BS16545-089. The agreement for all the stellar parameters and for the deduced $\log [\epsilon(\mathrm{Fe} \mathrm{I})]$ is very good, well within the uncertainties, except for $T_{\text {eff }}$ for the second star, where our value is somewhat lower than that adopted by them; this difference appears to arise, at least in part, from differences in the adopted photometry. They adopt the solar Fe abundance of Asplund et al. (2009), which is 0.07 dex higher than the one we use. Their Figure 3(c) compares their set of $W_{\lambda}$ from a 2008 HIRES/Keck spectrum with ours from a 2002 spectrum; there is a small offset of $\sim 3 \mathrm{~m} \AA$ with very small scatter.

Some of the brighter well-studied metal-poor stars observed as part of the Keck Pilot Project (e.g., BD 3-740, G139-8) have detailed abundance analyses in the literature. As was discussed in Cohen et al. (2002) and in Carretta et al. (2002), our results are in good overall agreement in these cases as well.

\section{ABUNDANCE RATIO OUTLIERS}

After all the abundance analyses were completed, we constructed plots of $[\mathrm{X} / \mathrm{Fe}]$ versus $[\mathrm{Fe} / \mathrm{H}]$ (Figures 5-9) based on the data in Table 10. We then selected from these plots the strong (i.e., very discrepant) outliers, either high or low, for verification of their outlier status. Each of these was checked in detail, looking for mistakes, inspecting the original spectra, remeasuring the equivalent widths of selected lines again if necessary, eliminating specific lines known to be blends, etc. This resulted in one of several outcomes: a correction to $[\mathrm{X} / \mathrm{Fe}]$ such that the star is no longer an outlier ${ }^{12}$, a verification of the outlier status

\footnotetext{
12 To avoid confusion, such stars are not specifically identified in the figures.
} 


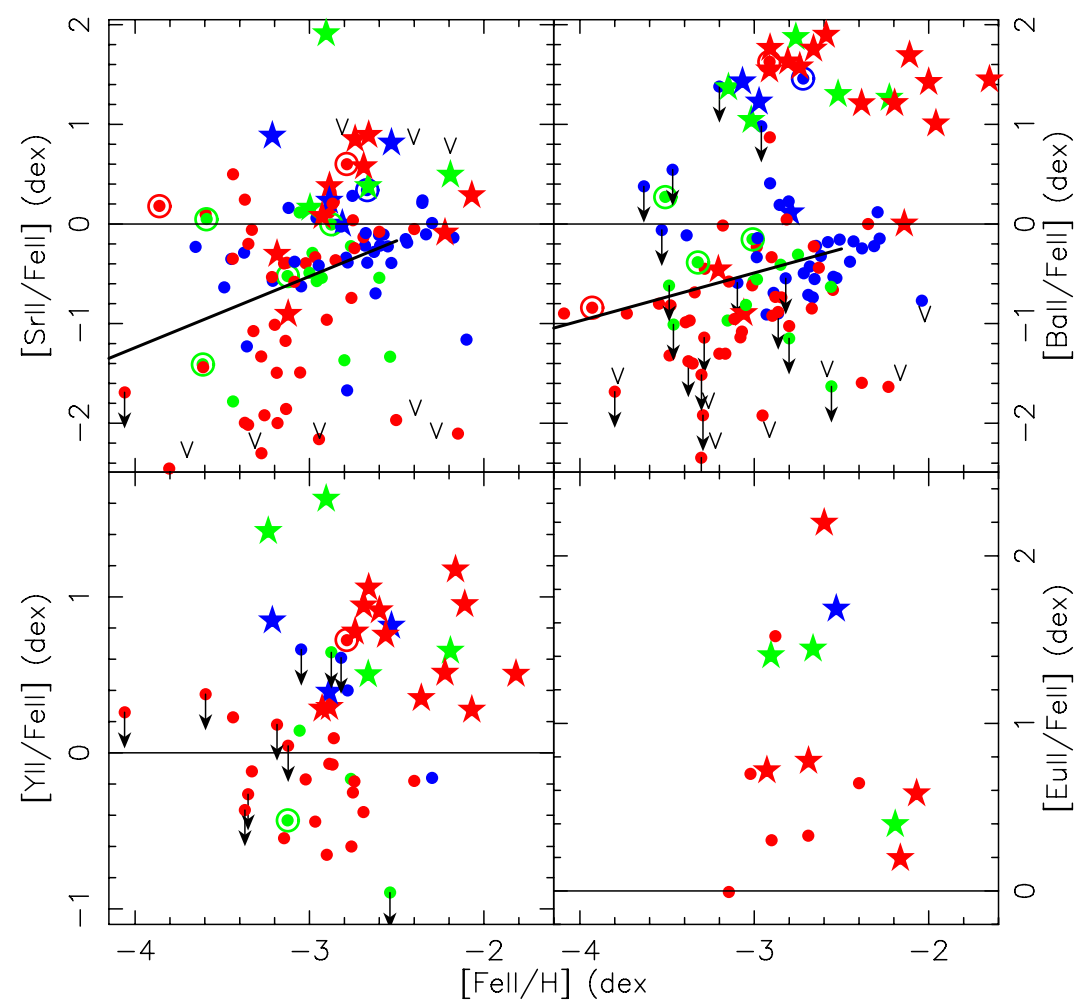

Figure 9. [Sr II/Fe] (upper left), [Ba II/Fe] (upper right), [Y II/Fe] (lower left), and [Eu II/Fe] (lower right) vs. [Fe/H] for the 0Z sample. Upper limits are ignored for Eu II. Symbols are as in Figure 3. Notes regarding checks of outliers are as in Figure 5. The linear fits from Table 13 are shown when available.

(A color version of this figure is available in the online journal.)

(indicated by "V" in the relevant figures for that species), or a decision that the data in hand were not adequate to determine whether or not the outlier status is correct; such cases are indicated by "?" in these figures. When possible, additional spectra were obtained to resolve the uncertain cases, either covering a wider spectral range or of higher $\mathrm{S} / \mathrm{N}$.

In the early stages of the $0 \mathrm{Z}$ project, we believed that several $\mathrm{C}$-stars were strong outliers for $[\mathrm{Ca} / \mathrm{Fe}]$. Our determination of the $\mathrm{Ca}$ abundance turned out to be problematic in those very C-rich stars whose spectra were obtained prior to HIRES detector upgrade in mid-2004 and thus included only a limited wavelength range. In an effort to derive $\mathrm{Ca}$ abundances from these early HIRES spectra, we ended up using lines which were crowded/blended, presumably by molecular features. This was only realized fairly recently (Cohen et al. 2012) when we obtained additional C-star HIRES spectra extending out to $8000 \AA$ which covered key Ca I lines in the $6160 \AA$ region. The $6160 \AA$ region is quite clean; it is not afflicted by $\mathrm{CH}, \mathrm{CN}$, or $\mathrm{C}_{2}$ bands. We found much lower (up to 0.75 dex lower) $\mathrm{Ca}$ abundances from the additional $\mathrm{Ca}$ lines in these $\mathrm{C}$-stars than those we previously published in Cohen et al. (2006). Fortunately our earlier paper does not focus at all on the Ca abundances in the EMP C-stars.

\subsection{Linear Fits to Abundance Ratios}

Based on the extensive previous work in this field (see, e.g., the discussion of toy models in Section 6 of Cohen \& Huang 2009), we chose to approximate the behavior of abundance ratios versus $[\mathrm{Fe} / \mathrm{H}]$ as a linear relationship in the EMP regime of our HES sample. We therefore fit lines to the abundance ratios $[\mathrm{X} / \mathrm{Fe}]$ versus $[\mathrm{Fe} / \mathrm{H}]$ where there is adequate data for species X. In order to demonstrate the slope of the line, Table 13 gives the evaluations of the line equation for each species $\mathrm{X}$ at

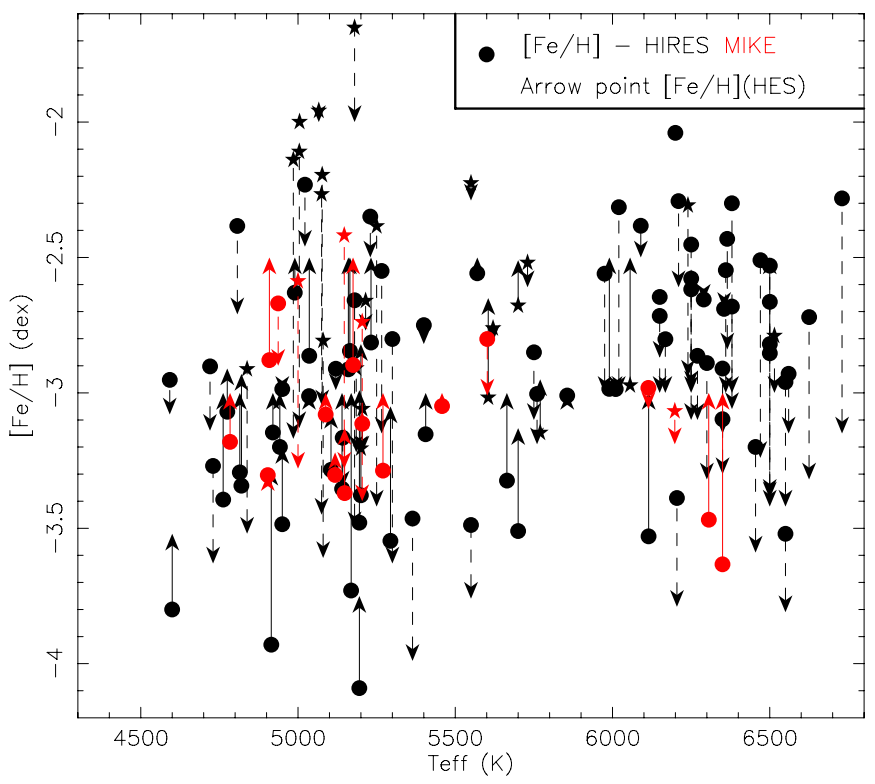

Figure 10. Difference between the $[\mathrm{Fe} / \mathrm{H}]$ deduced from the high resolution Keck and Magellan spectra, indicated by the filled circles (C-stars are denoted by small stars), and the moderate resolution follow up spectra analyzed with a modified version of the Beers et al. (1998) algorithm, indicated by the points of the arrows. If $[\mathrm{Fe} / \mathrm{H}](\mathrm{HIRES})>[\mathrm{Fe} / \mathrm{H}](\mathrm{HES})$, the arrow is shown as a solid line; otherwise it is drawn as a dashed line. For the hot dwarfs, which are on the right side of this figure, the arrows mostly point downward indicating that $[\mathrm{Fe} / \mathrm{H}](\mathrm{HIRES})>[\mathrm{Fe} / \mathrm{H}](\mathrm{HES})$.

(A color version of this figure is available in the online journal.)

$[\mathrm{Fe} / \mathrm{H}]=-3.5$ and -3.0 dex. For titanium, the line was fit to [Ti12/Fe12], which denotes the mean of $\left[\mathrm{Ti}_{\mathrm{I}} / \mathrm{Fe} \mathrm{I}\right]$ and [Ti II/Fe II]. The fit to [Ba II/Fe II] is not as well determined as some of the others because of the many upper limits for the Ba 
Table 13

Linear Fits for C-Normal Stars from the HES

\begin{tabular}{|c|c|c|c|c|c|}
\hline Element & $N($ stars $)$ & $\begin{array}{c}\text { At }[\mathrm{Fe} / \mathrm{H}]-3 \\
(\mathrm{dex})\end{array}$ & $\begin{array}{c}{[\mathrm{At} \mathrm{Fe} / \mathrm{H}]-3.5} \\
(\mathrm{dex})\end{array}$ & $\begin{array}{c}\sigma^{\mathrm{a}} \\
(\mathrm{dex})\end{array}$ & $\begin{array}{l}\text { Outliers }^{\mathrm{b}} \\
\text { High,Low }\end{array}$ \\
\hline \multicolumn{6}{|l|}{ All } \\
\hline$\left[\mathrm{Fe}_{\mathrm{I}} / \mathrm{Fe} \mathrm{II}\right]$ & 120 & 0.00 & 0.00 & 0.10 & \\
\hline [Ti I/Ti II] & 86 & 0.04 & 0.04 & 0.18 & \\
\hline Exclude & C-Stars & & & & \\
\hline$[\mathrm{Mg} / \mathrm{Fe}]$ & 81 & +0.37 & +0.37 & 0.17 & $2 \mathrm{H}, 1 \mathrm{~L}$ \\
\hline$[\mathrm{Al} / \mathrm{Fe}]$ & 81 & -0.16 & -0.13 & 0.24 & $1 \mathrm{H}, 1 \mathrm{~L}$ \\
\hline$[\mathrm{Si} / \mathrm{Fe}]^{\mathrm{c}}$ & 29 & +0.03 & -0.04 & 0.18 & none \\
\hline$[\mathrm{Si} / \mathrm{Fe}]^{\mathrm{d}}$ & 48 & +0.37 & +0.46 & 0.20 & $1 \mathrm{H}, 1 \mathrm{~L}$ \\
\hline$[\mathrm{Ca} / \mathrm{Fe}]$ & 81 & +0.27 & +0.27 & 0.15 & $3 \mathrm{~L}$ \\
\hline$[\alpha / \mathrm{Fe}]$ & 81 & +0.31 & +0.31 & 0.14 & $2 \mathrm{~L}, 2 \mathrm{H}$ \\
\hline$[\mathrm{Sc}$ II $/ \mathrm{Fe} \mathrm{I}]$ & 77 & +0.13 & +0.11 & 0.14 & $2 \mathrm{~L}, 2 \mathrm{H}$ \\
\hline [Ti II/Fe II] & 81 & +0.23 & +0.16 & 0.14 & $2 \mathrm{~L}$ \\
\hline [Ti12/Fe12] & 81 & +0.24 & +0.18 & 0.14 & $2 \mathrm{~L}$ \\
\hline$[\mathrm{Cr} / \mathrm{Fe}]$ & 80 & -0.35 & -0.47 & 0.13 & $2 \mathrm{H}$ \\
\hline$[\mathrm{Mn} / \mathrm{Fe}]$ & 70 & -0.71 & -0.83 & 0.16 & $5 \mathrm{H}, 2 \mathrm{~L}$ \\
\hline [Mn II/Fe II] & 23 & -0.44 & -0.49 & 0.18 & $2 \mathrm{H}$ \\
\hline$[\mathrm{Co} / \mathrm{Fe}]^{\mathrm{c}}$ & 19 & +0.40 & +0.47 & 0.15 & $2 \mathrm{UL}$ \\
\hline$[\mathrm{Co} / \mathrm{Fe}]^{\mathrm{d}}$ & 45 & +0.33 & +0.43 & 0.16 & $1 \mathrm{H}, 1 \mathrm{~L}$ \\
\hline$[\mathrm{Ni} / \mathrm{Fe}]$ & 68 & -0.08 & -0.02 & 0.21 & none \\
\hline$[\mathrm{Zn} / \mathrm{Fe}]^{\mathrm{e}, \mathrm{f}}$ & 20 & +0.37 & +0.37 & 0.25 & none \\
\hline$[\mathrm{Sr} \text { II } / \mathrm{Fe} \text { II }]^{\mathrm{e}}$ & 81 & -0.52 & -0.88 & 0.66 & $1 \mathrm{~L}, 1 \mathrm{H}$ \\
\hline$[\mathrm{Ba} \text { II } / \mathrm{Fe} \text { II }]^{\mathrm{e}}$ & 63 & -0.49 & -0.73 & 0.55 & 1L \\
\hline$\left[\mathrm{Ba} \mathrm{II} / \mathrm{Fe} \mathrm{II}^{\mathrm{g}}\right]^{\mathrm{s}}$ & 73 & -0.60 & -0.91 & 0.61 & $1 \mathrm{~L}$ \\
\hline
\end{tabular}

Notes.

${ }^{\mathrm{a}} \sigma$ of $[\mathrm{X} / \mathrm{Fe}]$ about the linear fit.

b Strong outliers with $[\mathrm{Fe} / \mathrm{H}]<-2.5$ dex excluded from fit.

c For hot dwarfs.

${ }^{\mathrm{d}}$ For cool giants.

e Upper limits are ignored.

${ }^{\mathrm{f}}$ Fit is very uncertain.

g Includes upper limits as detections for $T_{\text {eff }}<6000 \mathrm{~K}$.

abundance in the $0 \mathrm{Z}$ sample, which were ignored in constructing this fit. To at least partially take this into account, a second fit for $[\mathrm{Ba} / \mathrm{Fe}]$ versus $[\mathrm{Fe} / \mathrm{H}]$ was carried out assuming that the upper limits for $[\mathrm{Ba} / \mathrm{Fe}]$ in stars with $T_{\text {eff }}<6000 \mathrm{~K}$ are actually detections. This increases the sample size and slightly lowers the fit value at low Fe metallicities. The table also gives the mean and $\mathrm{rms}$ dispersion for $[\mathrm{Fe} / \mathrm{H}](\mathrm{Fe} \mathrm{I})-[\mathrm{Fe} / \mathrm{H}](\mathrm{Fe}$ II $)$, and the same for the more temperature dependent Ti. As noted earlier, the means of 0.0 independent of Fe-metallicity and the low $\sigma$, especially for Fe, where $\sigma=0.10$ dex, support the validity of our procedures and abundance analyses.

These fits exclude C-stars and also a small number of strong outliers (either high or low) as indicated in the last column of Table 13. Only stars with $[\mathrm{Fe} / \mathrm{H}]<-2.5 \mathrm{dex}$ are included. The histogram of deviations from the linear fit is shown for the set of stars from which each fit was constructed for $\mathrm{Mg}, \mathrm{Ca}, \mathrm{Cr}$, and $\mathrm{Sr}$ in Figure 11. The fit for each of $[\mathrm{Mg} / \mathrm{Fe}]$ and $[\mathrm{Ca} / \mathrm{Fe}]$ versus $[\mathrm{Fe} / \mathrm{H}]$ is approximately a constant ratio, $[\mathrm{Mg} / \mathrm{Fe}] \sim+0.40 \mathrm{dex}$, $[\mathrm{Ca} / \mathrm{Fe}] \sim+0.27$ dex.

Our linear fits to $[\mathrm{X} / \mathrm{Fe}]$ versus $[\mathrm{Fe} / \mathrm{H}]$ given in Table 13 have been compared with those of Cayrel et al. (2004), both evaluated at $[\mathrm{Fe} / \mathrm{H}]=-3.0$ dex. Overall the agreement is very good. With the correction of +0.15 dex for $[\mathrm{Mg} / \mathrm{Fe}]$ advocated by Bonifacio et al. (2009) to the results of Cayrel et al. (2004), 7 of the 10 fits for species in common agree to within 0.05 dex. The fits for $[\mathrm{Si} / \mathrm{Fe}]$ evaluated at $[\mathrm{Fe} / \mathrm{H}]-3.0$ dex differ by 0.08 dex, but this fit shows a strong dependence on $T_{\text {eff }}$, discussed in Section 6.2; see also Figure 6 . The fits for $[\mathrm{Ca} / \mathrm{Fe}]$ differ by

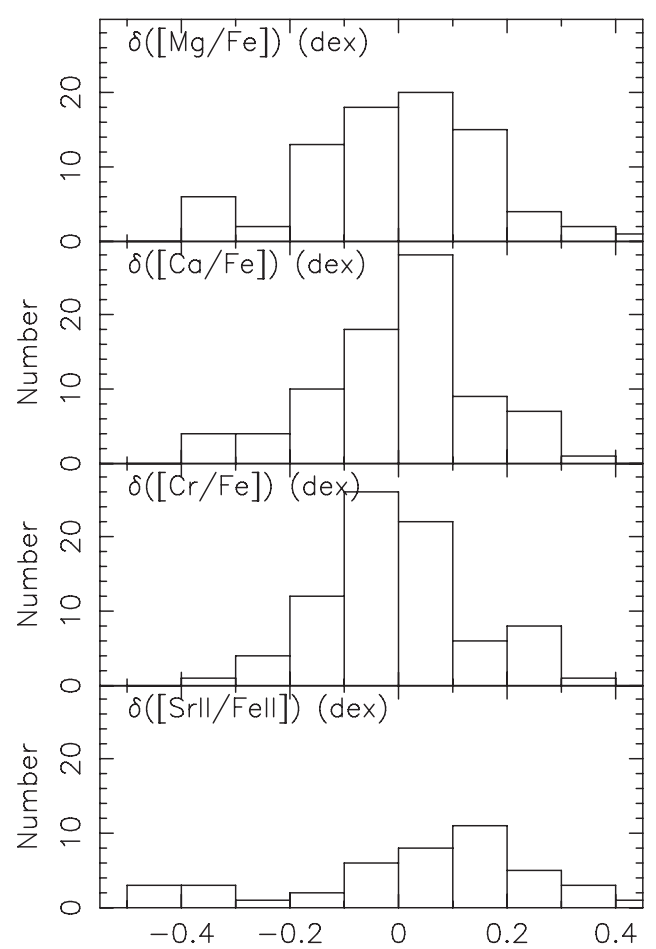

Figure 11. Deviations from the linear fits for $[\mathrm{Mg} / \mathrm{Fe}],[\mathrm{Ca} / \mathrm{Fe}],[\mathrm{Cr} / \mathrm{Fe}]$, and [Sr II/Fe II] (from top to bottom) are shown as histograms. C-stars, outliers, and stars with $[\mathrm{Fe} / \mathrm{H}]>-2.5$ dex were not used in the construction of the linear fits.

only $0.06 \mathrm{dex}$ at the nominal value of $[\mathrm{Fe} / \mathrm{H}]$, which is within the uncertainty of the comparison. The largest difference is only $0.09 \mathrm{dex}$ (and that is the fit for $[\mathrm{Zn} / \mathrm{Fe}]$, an element with at most two detectable weak lines for EMP star spectra in the optical wavelength regime). The dispersions around the fits from the First Stars project of Cayrel et al. (2004) are somewhat smaller than ours in most cases, perhaps because their sample is more homogeneous (in terms of $T_{\text {eff }}$ and $\log (g)$ ), and is considerably brighter in the mean, thus making it easier to get high $\mathrm{S} / \mathrm{N}$ high spectral resolution spectra.

The strong outliers from the mean relations of $[\mathrm{X} / \mathrm{Fe}]$ versus $[\mathrm{Fe} / \mathrm{H}]$, defined as those stars deviant by $\geqslant 5 \sigma$, are listed in Table 14. Given the very large spread in the abundance ratios of the heavy neutron capture elements $\mathrm{Sr}$ and $\mathrm{Ba}$, we require deviations of $10 \sigma$ for outlier status for these two species. Ignoring the C-stars, this leads to approximately $15 \%$ of the sample being strong outliers in one or more elements between $\mathrm{Mg}$ and $\mathrm{Ni}$. This rises to $\sim 19 \%$ if very strong $(\geqslant 10 \sigma)$ outliers for $\mathrm{Sr}$ and $\mathrm{Ba}$ are included. There is no question that they are statistically significantly different from the bulk of the population, and are not in the far wings of some smooth Gaussian distribution of errors. For our sample size, there should only be 1 star more deviant than $2.5 \sigma$, where $\sigma$ is the total observational uncertainty given in Table 5 (for dwarfs) or 4 (for giants), off the mean distribution in such relations for any given species.

As was first shown by McWilliam et al. (1995a), several of these linear fits have a statistically significant slope (e.g., $[\mathrm{Cr} / \mathrm{Fe}],[\mathrm{Mn} / \mathrm{Fe}],[\mathrm{Sr}, \mathrm{Fe}],[\mathrm{Ba} / \mathrm{Fe}]$, etc.). They begin with a large positive or negative value at the lowest $[\mathrm{Fe} / \mathrm{H}]$, then approach $[\mathrm{X} / \mathrm{Fe}]=0.0$ at some $[\mathrm{Fe} / \mathrm{H}]$ generally near $-2.5 \mathrm{dex}$. We assume that once the linear fit reaches the value of 0.0 dex, it remains at 0.0 for all higher $[\mathrm{Fe} / \mathrm{H}]$. This modification only affects the evaluation the deviation from the linear fits for strong 
Table 14

Strong Outliers Among the C-Normal Stars from the HES

\begin{tabular}{|c|c|c|c|}
\hline ID & $\begin{array}{c}{[\mathrm{X} / \mathrm{Fe}]} \\
(\mathrm{dex})\end{array}$ & $\begin{array}{l}\Delta[\mathrm{X} / \mathrm{Fe}]^{\mathrm{a}} \\
\quad(\mathrm{dex})\end{array}$ & $\Delta[\mathrm{X} / \mathrm{Fe}] / \sigma^{\mathrm{b}}$ \\
\hline \multicolumn{4}{|l|}{$\mathrm{Mg}$} \\
\hline HE0533-5340 & -0.234 & -0.64 & -7 \\
\hline HE $1012-1540^{c}$ & +1.38 & +0.98 & +11 \\
\hline $\mathrm{HE} 2323-0256^{\mathrm{c}}$ & +1.39 & +0.99 & +11 \\
\hline \multicolumn{4}{|l|}{$\mathrm{Al}$} \\
\hline HE0533-5340 & -0.84 & -0.65 & -6 \\
\hline $\mathrm{HE} 1012-1540^{\mathrm{c}}$ & +0.49 & +0.62 & +7 \\
\hline HE2200-1946 & +0.43 & +0.62 & +7 \\
\hline HE2251-0821 & +0.37 & +0.54 & +6 \\
\hline $\mathrm{HE} 2323-0256^{\mathrm{c}}$ & +0.41 & +0.51 & +6 \\
\hline \multicolumn{4}{|l|}{$\mathrm{Si}$} \\
\hline HE1424-0241 & -1.01 & -1.57 & -10 \\
\hline \multicolumn{4}{|l|}{$\mathrm{Ca}$} \\
\hline HE0349-0045 & -0.26 & -0.53 & -8 \\
\hline HE1424-0241 & -0.57 & -0.85 & -12 \\
\hline HE1500-1628d & -0.29 & -0.57 & -8 \\
\hline \multicolumn{4}{|l|}{$\mathrm{Sc}$} \\
\hline HE0132-2429 & +0.75 & +0.64 & +6 \\
\hline HE0218-2738 & +0.62 & +0.46 & +5 \\
\hline HE0533-5340 & -0.56 & -0.71 & -6 \\
\hline HE2215-2548 & -0.41 & -0.54 & -5 \\
\hline \multicolumn{4}{|l|}{ Ti12 } \\
\hline HE $1500-1628^{d}$ & -0.28 & -0.66 & -6 \\
\hline \multicolumn{4}{|l|}{$\mathrm{Cr}$} \\
\hline HE2344-2800 & +0.08 & +0.36 & +5 \\
\hline \multicolumn{4}{|l|}{$\mathrm{Mn}$} \\
\hline HE0122-1616 & -1.23 & -0.57 & -6 \\
\hline HE1226-1149 & -0.04 & +0.64 & +7 \\
\hline HE1245-0215 & -0.10 & +0.58 & +6 \\
\hline HE1424-0241 & -0.26 & +0.72 & +8 \\
\hline HE2215-2548 & -1.51 & -0.80 & -9 \\
\hline HE2344-2800 & -0.01 & +0.62 & +7 \\
\hline \multicolumn{4}{|l|}{ MnII } \\
\hline HE1424-0241 & +0.23 & +0.77 & +6 \\
\hline HE2344-2800 & +0.46 & +0.86 & +7 \\
\hline \multicolumn{4}{|l|}{ Co } \\
\hline HE0533-5340 & -0.42 & -0.77 & -11 \\
\hline $\mathrm{HE} 1424-0241^{\mathrm{e}}$ & +1.00 & +0.44 & +6 \\
\hline \multicolumn{4}{|l|}{$\mathrm{Zn}^{\mathrm{f}}$} \\
\hline HE0349-0045 d & -0.31 & -0.65 & -5 \\
\hline \multicolumn{4}{|l|}{$\mathrm{Sr}^{\mathrm{h}}$} \\
\hline HE0349-0045 & -2.02 & $-2.02^{\mathrm{g}}$ & -13 \\
\hline HE1422-0818 & -2.28 & -1.55 & -10 \\
\hline HE $1500-1628^{d}$ & -2.09 & $-2.09^{\mathrm{g}}$ & -13 \\
\hline HE2249-1704 & -2.15 & -1.66 & -10 \\
\hline \multicolumn{4}{|l|}{$\mathrm{Ba}^{\mathrm{i}}$} \\
\hline HE0305-5442 ${ }^{\mathrm{i}}$ & -2.41 & -1.77 & $\leqslant-15$ \\
\hline HE0349-0045 & -1.56 & $-1.56^{\mathrm{g}}$ & -13 \\
\hline HE0447-1858 ${ }^{\mathrm{i}}$ & -1.61 & -1.33 & $\leqslant-11$ \\
\hline HE1422-0818 ${ }^{i}$ & -1.90 & -1.27 & $\leqslant-11$ \\
\hline HE $1500-1628^{d}$ & -1.72 & -1.52 & -13 \\
\hline HE2249-1704 & -1.92 & -1.45 & -12 \\
\hline HE2251-0821 & +1.76 & +2.21 & +18 \\
\hline
\end{tabular}

Notes.

a The difference between $[\mathrm{X} / \mathrm{Fe}]$ and the linear fit for $[\mathrm{X} / \mathrm{Fe}]$ from Table 13 evaluated at the $[\mathrm{Fe} / \mathrm{H}]$ of the star.

b The ratio of $\Delta[\mathrm{X} / \mathrm{Fe}]$ to $\sigma$ for a single star given in the second column of Table 4 .

c This star is not "C-normal."

${ }^{\mathrm{d}}[\mathrm{Fe} / \mathrm{H}]>-2.5 \mathrm{dex}$, not used in constructing linear fits.

e The trend of $[\mathrm{Co} / \mathrm{Fe}]$ versus $[\mathrm{Fe} / \mathrm{H}]$ is not as well established as for other species; these stars may be at the extremes of the trends rather than genuine outliers.

${ }^{\mathrm{f}}$ The trend of $[\mathrm{Zn} / \mathrm{Fe}]$ versus $[\mathrm{Fe} / \mathrm{H}]$ is not as well established as for other species and at best only two weak $\mathrm{Zn}$ I lines are detected in our spectra.

g Fit value set to 0.0 dex. See Section 8.1.

${ }^{\mathrm{h}} \sigma$ includes extra term discussed in Section 8.1, and is $0.16 \mathrm{dex}$ for $[\mathrm{Sr} / \mathrm{Fe}]$ and 0.12 dex for $[\mathrm{Ba} / \mathrm{Fe}]$.

${ }^{\mathrm{i}}$ Upper limit to [Ba II/Fe II], not used in constructing fit.
Table 15

Dispersion About Linear Fit versus Observational Uncertainty for C-normal Stars

\begin{tabular}{lccc}
\hline \hline Element & $\begin{array}{c}\sigma(\text { Lin. Fit }) \\
(\text { dex })\end{array}$ & $\begin{array}{c}\text { Pred. } \sigma \\
(\text { dex })\end{array}$ & Ratio \\
\hline$[\mathrm{Mg} / \mathrm{Fe}]$ & 0.17 & 0.09 & 1.9 \\
{$[\mathrm{Al} / \mathrm{Fe}]$} & 0.24 & 0.09 & 2.7 \\
{$[\mathrm{Si} / \mathrm{Fe}]^{\mathrm{a}}$} & 0.18 & 0.16 & 1.2 \\
{$[\mathrm{Si} / \mathrm{Fe}]^{\mathrm{b}}$} & 0.20 & 0.16 & 1.2 \\
{$[\mathrm{Ca} / \mathrm{Fe}]$} & 0.15 & 0.07 & 2.1 \\
{$[\mathrm{Sc} \mathrm{II} / \mathrm{Fe} \mathrm{I}]$} & 0.14 & 0.11 & 1.3 \\
{$[\mathrm{Ti}$ II $/ \mathrm{Fe} \mathrm{II}]$} & 0.14 & 0.09 & 1.6 \\
{$[\mathrm{Cr} / \mathrm{Fe}]$} & 0.13 & 0.07 & 1.9 \\
{$[\mathrm{Mn} / \mathrm{Fe}]$} & 0.16 & 0.09 & 1.8 \\
{$[\mathrm{Ni} / \mathrm{Fe}]$} & 0.21 & 0.17 & 4.1 \\
{$[\mathrm{Sr}$ II $/ \mathrm{Fe} \mathrm{II}]$} & 0.66 & $0.16^{\mathrm{c}}$ & 4.6 \\
{$[\mathrm{Ba}$ II $/ \mathrm{Fe} \mathrm{II}]$} & 0.55 & $0.12^{\mathrm{c}}$ & \\
\hline
\end{tabular}

Notes.

${ }^{a}$ Fit for dwarfs.

b Fit for giants.

${ }^{c}$ Uses augmented $\sigma$ with term from slope, 0.16 dex for [Sr II/Fe II] and 0.12 dex for [Ba II/Fe II].

outliers in $[\mathrm{Sr} / \mathrm{Fe}]$ and in $[\mathrm{Ba} / \mathrm{Fe}]$ at metallicities near the high end of the $0 \mathrm{Z}$ sample, i.e., those higher than -2.5 dex.

There are several stars which are very deviant low outliers in $[\mathrm{Ba} / \mathrm{Fe}]$. One of these (HE0305-5442) has an upper limit to $[\mathrm{Ba} / \mathrm{H}]$ which is 0.3 dex lower than that of the previous record holder for the lowest known [Ba/H], Draco 119 (Fulbright et al. 2004), which also has only an upper limit.

We compare the dispersion we measured around the linear fits for the restricted sample of stars used to construct each fit for various elements with those based on the predicted uncertainties in Table 15. In doing so, we have assumed that the uncertainty in the $[\mathrm{Fe} / \mathrm{H}]$ values does not contribute significantly to that of the abundance ratios $[\mathrm{X} / \mathrm{Fe}]$, which is not always valid. Hence there is another term contributing to the dispersion around the linear fits, which can be approximated as $\sigma(x) \frac{d y}{d x}$, where $x$ is $[\mathrm{Fe} / \mathrm{H}]$ and $y$ is $[\mathrm{X} / \mathrm{Fe}]$. For those species with significant slopes for the linear fits given in Table 13, this term will increase the observed dispersion about the linear fits compared to the one predicted based solely the analysis and measurement errors in $[\mathrm{X} / \mathrm{Fe}]$. This extra term only affects those elements with the largest slopes, which are, as shown in Table 13, Sr II and Ba II; it raises the expected dispersions of $[\mathrm{Sr} / \mathrm{Fe}]$ and of $[\mathrm{Ba} / \mathrm{Fe}]$ from 0.12 and 0.10 dex, respectively, to 0.16 and 0.12 dex, respectively. We may have slightly underestimated the increase in the predicted dispersion for $[\mathrm{Ba} / \mathrm{Fe}]$ due to the many upper limits in the $\mathrm{Ba}$ abundance among our sample stars.

Such a comparison of the predicted and actual deviations around the linear fits to $[\mathrm{X} / \mathrm{Fe}]$ versus $[\mathrm{Fe} / \mathrm{H}]$ for species $\mathrm{X}$, shown in Table 15, suggests that, ignoring major outliers, there is no detectable range in $[\mathrm{X} / \mathrm{Fe}]$ versus $[\mathrm{Fe} / \mathrm{H}]$ that is larger than the uncertainties for $\mathrm{Si}, \mathrm{Sc}$, and $\mathrm{Ni}$. The ratio of that measured (see Table 13) to that predicted (see Table 5 for dwarfs and Table 4 for giants), ranges from 1.1 to 1.3 for these three elements (see Table 15). The ratios for $\mathrm{Mg}, \mathrm{Ti}, \mathrm{Cr}$, and $\mathrm{Mn}$, are only slightly higher, 1.6 to 1.9 . They are still larger for $\mathrm{Al}$ (2.7), and $\mathrm{Ca}$ (2.1), while the heavy neutron capture elements $\mathrm{Sr}$ and $\mathrm{Ba}$ have the largest ratios $(\sim 4)$, hence the highest intrinsic dispersion, of all the species tested. 


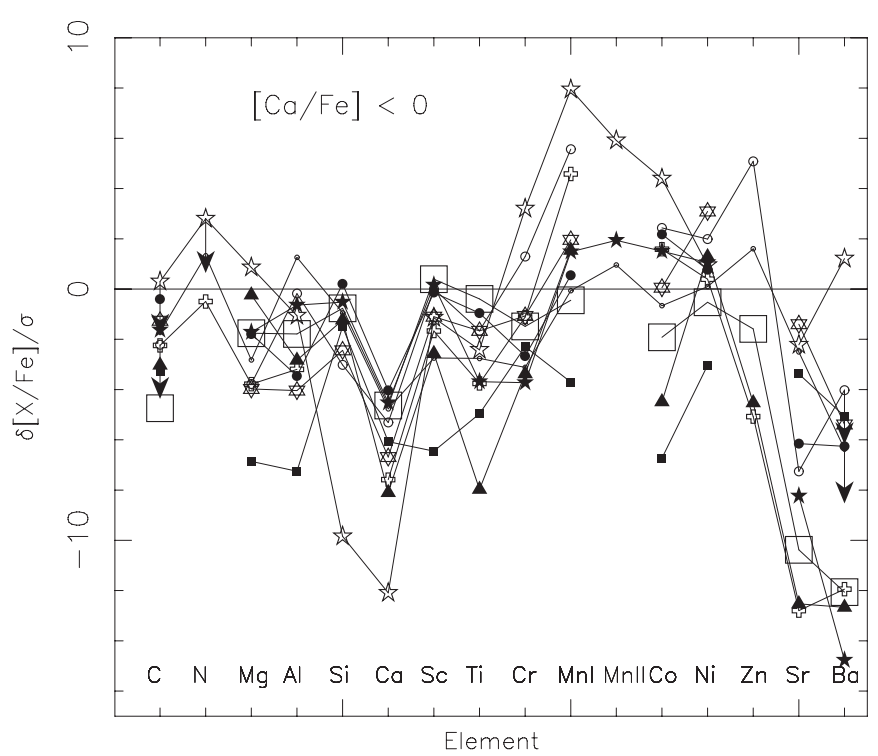

Figure 12. $\delta[\mathrm{X} / \mathrm{Fe}] / \sigma$ for $10 \mathrm{EMP}$ stars in our sample with sub-solar $[\mathrm{Ca} / \mathrm{Fe}]$, where $\delta[\mathrm{X} / \mathrm{Fe}] / \sigma$ is the difference between the derived value of $[\mathrm{X} / \mathrm{Fe}]$ and that from the linear fit to $[\mathrm{X} / \mathrm{Fe}]$ vs. $[\mathrm{Fe} / \mathrm{H}]$ using the derived Fe-metallicity of the star divided by a generous estimate of the uncertainty in the abundance ratio. A different symbol is used for each of the 10 stars.

\subsection{Low $[\mathrm{Ca} / \mathrm{Fe}]$ Stars}

A substantial number of stars with $[\mathrm{Ca} / \mathrm{Fe}]$ below the solar ratio (the "low-Ca" stars) are found in the present sample, a phenomenon that is virtually non-existent among solar neighborhood stars and Galactic disk stars, which are considerably closer and with higher metallicities than the $0 \mathrm{Z}$ sample. Low -Ca stars are also never seen among Galactic globular cluster stars, which are a population with characteristics closer to our $0 \mathrm{Z}$ sample. As is shown in Figure 6, there are $10 \mathrm{C}$-normal stars, as well as $1 s$-process enhanced $\mathrm{C}$-star with a high $[\mathrm{Fe} / \mathrm{H}]$ (HE2353-1758) which we ignore, with $[\mathrm{Ca} / \mathrm{Fe}]$ below the solar ratio. Table 13 gives the mean value of $[\mathrm{Ca} / \mathrm{Fe}]$ for $\mathrm{C}$-normal stars in our sample as +0.27 dex independent of $[\mathrm{Fe} / \mathrm{H}]$. Note that there are no $\mathrm{C}$-normal stars with $[\mathrm{Ca} / \mathrm{Fe}]>+0.54 \mathrm{dex}$, which would be the comparable requirement for "high-Ca" stars.

The minimum value for $[\mathrm{Ca} / \mathrm{Fe}]$ in our sample is $-0.6 \mathrm{dex}$ for HE1424-0241, an extremely peculiar star described in detail in Cohen et al. (2007). However, only one of the low$\mathrm{Ca}$ stars has $[\mathrm{Mg} / \mathrm{Fe}]$ below the solar ratio (see Figure 5), while HE1424-0241 has the normal EMP halo star value of +0.44 dex, and only HE1424-0241 has $[\mathrm{Si} / \mathrm{Fe}]<-0.07 \mathrm{dex}$ (with an extremely low value of -1.0 dex).

Figure 12 displays the chemical inventory of the family of 10 stars (all giants ${ }^{13}$ from our sample with $[\mathrm{Ca} / \mathrm{Fe}]<0$ dex. The vertical axis is $\delta[\mathrm{X} / \mathrm{Fe}] / \sigma$, where the numerator of this expression is the deviation from the linear fit to $[\mathrm{X} / \mathrm{Fe}]$ versus $[\mathrm{Fe} / \mathrm{H}]$ at the Fe metallicity of the star. The denominator is the predicted observational uncertainty given in Tables 5 and 4, with $\sigma$ is set to 0.2 dex for $\mathrm{C}$ (from the $G$ band of $\mathrm{CH}$ ) and $\mathrm{N}$ from the $3360 \AA$ band of $\mathrm{NH}$. (The $\sigma$ values used for $\mathrm{Mg}, \mathrm{Si}$, and $\mathrm{Ca}$ for giants are $0.09,0.16$, and $0.11 \mathrm{dex}$, respectively.) Low $[\mathrm{Ca} / \mathrm{Fe}]$ is associated with low $[\mathrm{Mg}, \mathrm{Si} / \mathrm{Fe}]$, but the relative depletion of $\mathrm{Mg}$ and of $\mathrm{Si}$ (with the exception of one very anomalous star with very deficient $\mathrm{Si}$ ) measured in units of $\sigma$ is less than half the size of the Ca deficiency. If we attempt to compare

\footnotetext{
13 One star of these 10 low-Ca stars has $T_{\text {eff }} 2 \mathrm{~K}$ higher than the upper limit we allow for giants.
}

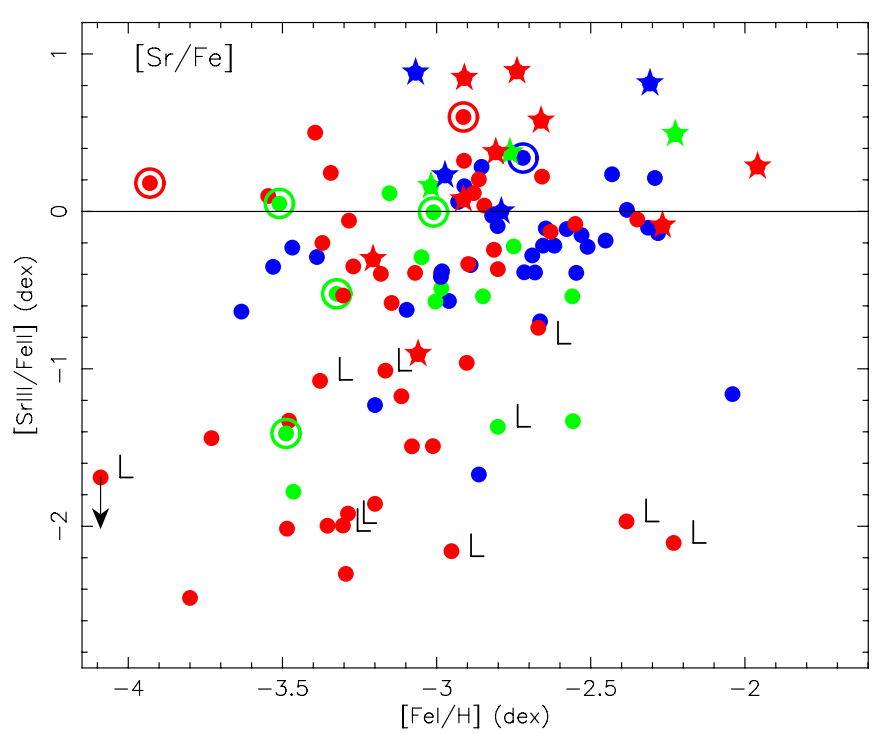

Figure 13. [ $\mathrm{Sr}$ II $/ \mathrm{Fe}$ II] vs. [Fe/H] with the stars with sub-solar $[\mathrm{Ca} / \mathrm{Fe}]$ marked by the letter "L." Note that the low-Ca family also has low heavy-neutron capture elements, well below the mean of our 0Z sample. Symbols are as in Figure 3. A typical error bar is shown for a single star.

(A color version of this figure is available in the online journal.)

the number of low outliers for $\mathrm{Mg}$ with those for $\mathrm{Ca}$, there are three stars with $[\mathrm{Mg} / \mathrm{Fe}]<0$ dex, of which the lowest $[\mathrm{Mg} / \mathrm{Fe}]$ is -0.24 dex $(\mathrm{HE} 0533-5340)$, while the lowest $[\mathrm{Ca} / \mathrm{Fe}]$ is -0.57 dex (HE1424-0241). However, since the mean value for $[\mathrm{Mg} / \mathrm{Fe}]$ is 0.10 dex higher than that of $[\mathrm{Ca} / \mathrm{Fe}]$, perhaps the relevant number for comparison is that there are 12 stars with $[\mathrm{Mg} / \mathrm{Fe}]<+0.10$ dex, 1 of which is a C-star, but only 4 of them are among those with sub-solar [Ca/Fe].

Sub-solar ratios of $[\mathrm{Ca} / \mathrm{Fe}]$ are also clearly associated with abnormally low $[\mathrm{Sr} / \mathrm{Fe}]$ and $[\mathrm{Ba} / \mathrm{Fe}]$ (see Figure 12 and also Figure 13). The low-Ca stars tend to lie along the lower envelope of the $[\mathrm{Sr} / \mathrm{Fe}]$ and $[\mathrm{Ba} / \mathrm{Fe}]$ versus $[\mathrm{Fe} / \mathrm{H}]$ distributions shown in the two upper panels of Figure 9, but not all the anomalously low neutron-capture element stars have low $[\mathrm{Ca} / \mathrm{Fe}]$. A check of the two stars with the lowest [Sr II/Fe II] in our sample (see Figure 9), neither of which has low-Ca, does not show any obvious anomalies among the lighter elements. A few of the low-Ca stars have high $[\mathrm{Mn} / \mathrm{Fe}]$ for their Fe-metallicity. Honda et al. (2011) have found a star (BS16920-016) with unusually high $[\mathrm{Zn} / \mathrm{Fe}]$, close to normal $[\mathrm{Mg} / \mathrm{Fe}]$, slightly low $[\mathrm{Si} / \mathrm{Fe}]$ and $[\mathrm{Ca} / \mathrm{Fe}]$, although not sub-solar $[\mathrm{Ca} / \mathrm{Fe}]$, and high $[\mathrm{Mn}, \mathrm{N} / \mathrm{Fe}]$, accompanied by a deficiency of the heavy neutroncapture elements, which may be related to this group of 10 low-Ca EMP stars we have isolated from our sample.

Differential analyses of large samples of solar-neighborhood dwarfs within a small range of $T_{\text {eff }}$ in the thin disk of the Galaxy as compared to stars in the thick disk such as those of Edvardsson et al. (1993), Bensby et al. (2005), Reddy et al. (2006), and Nissen \& Schuster (2010) have been able to achieve very high precision. These surveys have demonstrated that the trends of $[\mathrm{X} / \mathrm{Fe}]$ versus $[\mathrm{Fe} / \mathrm{H}]$ are close but not identical between the various disk stellar populations of the Galaxy; the trends of the various disk components are separated by not more than 0.15 dex, a small difference not easy to detect in the present sample. However, in all such studies to date, sub-solar values of the $\alpha$-element abundance ratios (i.e., of $[\mathrm{Mg}, \mathrm{Si}$, or $\mathrm{Ca} / \mathrm{Fe}]$ ) are never seen. This also holds for the First Stars survey of EMP giants (Cayrel et al. 2004), whose sample is in the mean 
$\sim 2.5$ mag brighter than ours; there are no stars with sub-solar $[\mathrm{Mg}, \mathrm{Si}$, or $\mathrm{Ca} / \mathrm{Fe}]$ in the First Stars survey; there are also no stars with sub-solar $[\mathrm{Ca} / \mathrm{Fe}]$ in the pioneering study of McWilliam et al. (1995a).

A few moderately metal-poor halo field stars have been found that appear to be $\alpha$-poor. Fulbright (2002) suggested that lower $[\alpha / \mathrm{Fe}]$ stars are found among those with high space velocities with respect to the local standard of rest, while Stephens \& Boesgaard (2002) suggest such stars are associated with the outer halo. The most extreme $\alpha$-poor stars, including that found by Carney et al. (1997), were reviewed by Ivans et al. (2003). These stars show depletions of $\mathrm{Na}, \mathrm{Mg}, \mathrm{Al}, \mathrm{Si}, \mathrm{Ca}$, and $\mathrm{Sc}$ with respect to $\mathrm{Fe}$, with $\mathrm{Sr}, \mathrm{Y}$, and $\mathrm{Ba}$ also anomalously low. The explanation offered by Ivans et al. (2003) is that their chemical inventory has a composite origin, with SNIa contributing a substantial fraction $(>1 / 3)$ of their chemical inventory, and SNII the rest. We calculate a rough guess of this value for the Sun, assuming that the SNII contribution has the mean $[\mathrm{Mg} / \mathrm{Fe}]$ of our $0 Z$ sample, $+0.37 \mathrm{dex}$, and that SNIa contribute Fe, but no $\mathrm{Mg}$; the result is that SNIa contribute $\sim 30 \%$ of the Sun's total chemical inventory. Given that, how such a substantial enhancement of the relative contribution of SNIa at such low metallicities as are being considered among the $0 \mathrm{Z}$ stars could occur is quite unclear.

Only the prompt SNIa contribution is relevant for the chemical inventory of EMP stars. What distinguishes the prompt SNIa from the more numerous delayed SNIa is not clear (Greggio et al. 2008). Initial calculations of SNIa nucleosynthesis yields were carried out by Nomoto et al. (1984). The set of calculations often used today are those of Iwamoto et al. (1999), with more recent multi-dimensional calculations by Travaglio et al. (2004b). These strongly suggest that $\mathrm{Mg}$, given that $\mathrm{Mg}$ is produced almost entirely by SNII, should be even more depleted than Ca. However, this is not the case among the EMP stars studied here, although that does hold, at least roughly, for the sample collected by Ivans et al. (2003).

If one tries to use the difference between hydrostatic and explosive $\alpha$-burning nucleosynthesis invoked in Cohen et al. (2007), this too fails, as while $\mathrm{Mg}$ is produced in hydrostatic $\alpha$-burning, both $\mathrm{Si}$ and $\mathrm{Ca}$ are produced by explosive $\alpha$-burning, but $[\mathrm{Si} / \mathrm{Fe}]$ is closer to normal in all but one of these stars. Any proposed explanation for the low-Ca EMP stars must also address the accompanying depletion of the heavy neutron capture elements.

Slightly sub-solar $[\mathrm{Ca} / \mathrm{Fe}]$ is found in the stellar populations of several of the dSph satellites of the Milky Way, but only at the high Fe metallicity end of their abundance distributions, and also in those Galactic globular clusters Pal 12 (Cohen 2004) and Rup 106 (Brown et al. 1997) believed to have originally been part of the Sgr dSph galaxy, again with $[\mathrm{Fe} / \mathrm{H}]$ considerably above that characteristic of our $0 \mathrm{Z}$ sample. Examples of this are shown in Tolstoy et al. (2009) and in Kirby et al. (2011). These stars, however, show the typical SNIa pattern of depleted $\alpha$-elements, reaching down to -0.3 dex for $[\mathrm{Ca} / \mathrm{Fe}]$ and -0.2 dex for $[\mathrm{Mg} / \mathrm{Fe}]$ in Fornax (see, e.g., Figure 11 of Tolstoy et al. 2009), and have $[\mathrm{Fe} / \mathrm{H}]>-1.1 \mathrm{dex}$. They also have highly enhanced $[\mathrm{Ba} / \mathrm{Fe}]$, presumably arising from the $s$-process in AGB stars, through which the SNIa progenitors must pass prior to reaching the white dwarf regime.

Tolstoy et al. (2009) suggest that the last stage of chemical evolution in Fornax accompanies a dwindling star formation rate (SFR) before star formation ceases altogether. When the SFR is very low, the ratio of Type Ia to Type II SNe is large, producing sub-solar ratios for $[\alpha / \mathrm{Fe}]$. AGB stars within the aging population will continuously produce $s$-process elements, leading to highly-enhanced $[\mathrm{Ba} / \mathrm{Fe}]$, a characteristic not manifest in the EMP low-Ca population.

Our view of these various anomalies, and in particular of the Ca-poor nature of $\sim 10 \%$ of our $0 \mathrm{Z}$ sample of stars, is that early SNII must have been more diverse in their ejecta abundances than current models such as those of Woosley \& Weaver (1995) or more recently those of Kobayashi et al. (2006), based on the yields of Nomoto et al. (2006), or of Tominaga et al. (2007) or Heger \& Woosley (2010) suggest to date if they are the ultimate cause of what we have observed. As the above studies of SNII behavior indicate, the progenitor mass, the mass cut of ejection, the fallback, and other details of the SN can profoundly affect the chemical composition of the ejected material, and are difficult to model.

Several of the model SNII yield grids suggest that heavier progenitors produce more $\mathrm{O}$ and $\mathrm{Mg}$ (i.e., hydrostatic $\alpha$-elements) with respect to $\mathrm{Ca}$ and $\mathrm{Ti}$ (explosive $\alpha$-elements) than do lower mass progenitors. The models of Nomoto et al. (2006) predict a sizable increase (a factor of $\sim 18$ ) in the $\mathrm{Mg} / \mathrm{Ca}$ ratio in the ejecta for progenitor masses increasing from 13 to $30 M_{\odot}$. This suggests that a bias of the initial mass function (IMF) toward higher mass stars on the upper main sequence might also be relevant in producing the low-Ca EMP stars.

Cohen \& Kirby (2012) recently demonstrated that a group of stars with a very peculiar chemical inventory exists in the extreme outer halo Galactic globular cluster NGC 2419. The most obvious symptom is a very large deficiency of $\mathrm{Mg}$ (a factor of 5 compared to the bulk of the cluster stars). This is accompanied by very high $\mathrm{K}$, somewhat high $\mathrm{Si}$ and $\mathrm{Ca}$, and high Sc. The Fe-peak elements are constant in abundance for all the stars within this globular cluster. This too, while very peculiar, is not related to the low-Ca population we have isolated within the OZ EMP halo field stars.

\subsection{Other Smaller Outlier Families}

There are two other families of outliers we recognize within our sample. The first is a group of two EMP stars, both with $[\mathrm{Fe} / \mathrm{H}]<-3.5$ dex, and both highly C-enhanced (HE1012-1540 and HE2323-0256), although neither shows $\mathrm{C}_{2}$ bands. Multiple measurements of $v_{r}$ from high-dispersion spectra exist for each of these two stars. Unlike the higher-Fe-metallicity C-stars with $\mathrm{C}_{2}$ bands and in most cases $s$-process enhancements, Table 2 offers no evidence that either HE1012-1540 or HE2323-0256 is a binary. Their chemical inventory is shown in Figure 14. It is characterized by high $\mathrm{C}$ and $\mathrm{N}$, carrying on into high $\mathrm{Na}, \mathrm{Mg}$, and/or $\mathrm{Al}$, with more or less normal abundances for $\mathrm{Ca}$ and for the $\mathrm{Fe}$-peak elements. $[\mathrm{Sr} / \mathrm{Fe}]$ and $[\mathrm{Ba} / \mathrm{Fe}]$ are close to the solar value, which is quite high for such metal-poor stars. Although the higher metallicity $\mathrm{C}$-rich stars clearly have $\mathrm{C}>\mathrm{O}$, a tentative detection of $\mathrm{O}$ based on two UV OH lines in HE1012-1540 suggests that $\mathrm{O}$ is also highly enhanced in HE1012-1540; the upper limits from the $6300 \AA$ and $7770 \AA$ lines are consistent with this value. Assuming the UV line equivalent widths are valid, then $\epsilon(\mathrm{C}) / \epsilon(\mathrm{O}) \sim 1 / 2$. The issue of the oxygen abundance in the extremely low metallicity $\mathrm{C}$-rich stars requires additional attention and future observations. The origin of these stars is discussed in more detail in Section 9, where it is suggested that they are the continuation of the classical C-stars with $\mathrm{C}_{2}$ bands to still lower $[\mathrm{Fe} / \mathrm{H}]$; see Norris et al. (2013b) for a different interpretation. 


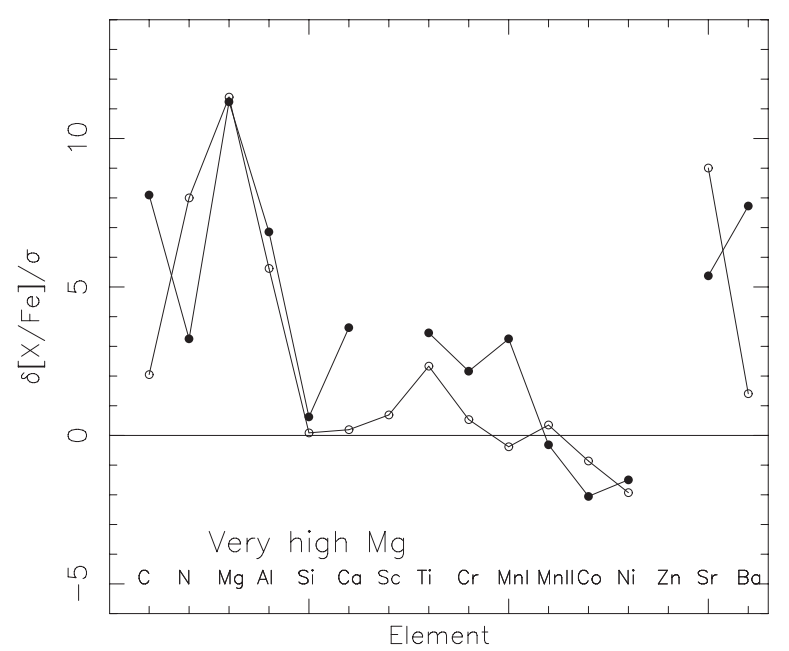

Figure 14. Same as Figure 12 for 2 EMP stars in our sample with very high $[\mathrm{Mg} / \mathrm{Fe}]$.

There is also is a small group of stars with peculiarities in only a small number of Fe-peak elements exemplified by HE2344-2800, shown in Figure 15. This subgiant has high $[\mathrm{Cr} / \mathrm{Fe}]$ and $[\mathrm{Mn} / \mathrm{Fe}]$ (measured from both $\mathrm{Mn}$ I and $\mathrm{Mn}$ II lines), but everything else is more or less normal. There are also a number of other stars which tend to have low heavy-neutron capture abundance ratios without having any of the peculiarities discussed above.

The families of outliers discussed above include all of the major outliers we have found.

\subsection{Mixed Stars}

Among the C-normal stars in our 0Z sample, we have found only limited signs of internal mixing of material that has modified the surface composition of the giants in our sample. Spite et al. (2006) detected in the First Stars project sample the expected variation of the ${ }^{12} \mathrm{C} /{ }^{13} \mathrm{C}$ ratio, which is a very sensitive diagnostic of such mixing, showing a correlation with $[\mathrm{C} / \mathrm{Fe}]$ and an anti-correlation with $[\mathrm{N} / \mathrm{Fe}]$. The $\mathrm{S} / \mathrm{N}$ of our spectra in the region of the $G$ band of $\mathrm{CH}$ is not generally adequate to reliably measure the weak ${ }^{13} \mathrm{CH}$ features.

Although not believed to arise from internal mixing, we see no sign of widespread anti-correlations between $\mathrm{Mg}$ and $\mathrm{Al}$ or $\mathrm{Mg}$ and $\mathrm{Na}$ such as are common among globular cluster stars, as described in the review by Gratton et al. (2004), where various theories for their origin are discussed. Since these are common in globular cluster stars, they are presumably present in at least some of those halo field stars which were formerly in globular clusters. The cool giant HE2217-4053 has high $\mathrm{Na}$ $([\mathrm{Na} / \mathrm{Fe}]+0.81$ dex $)$ and $\mathrm{Al}$ relative to $\mathrm{Fe}$, with $\mathrm{Mg}$ relatively low $([\mathrm{Mg} / \mathrm{Fe}]+0.06 \mathrm{dex})$, which is the classical pattern seen in such cases. Two other cool giants, HE2200-1946 and HE2339-5105, also show unusually high $[\mathrm{Na} / \mathrm{Fe}]$ but normal $[\mathrm{Mg} / \mathrm{Fe}]$. These all have very low $E(B-V)$, less than $0.02 \mathrm{mag}$, and are quite cool, so even though the $v_{r}$ for two of these are close to $0 \mathrm{~km} \mathrm{~s}^{-1}$, the $\mathrm{NaD}$ lines cannot be substantially enhanced by interstellar contributions. Ignoring $\mathrm{C}$-rich stars, only cool giants have $[\mathrm{Na} / \mathrm{Fe}]>+0.3$ dex.

\section{CARBON ENHANCED EMP STARS}

There are a substantial number of C-rich stars in our sample. Several subclasses of these stars have been defined by Beers $\&$

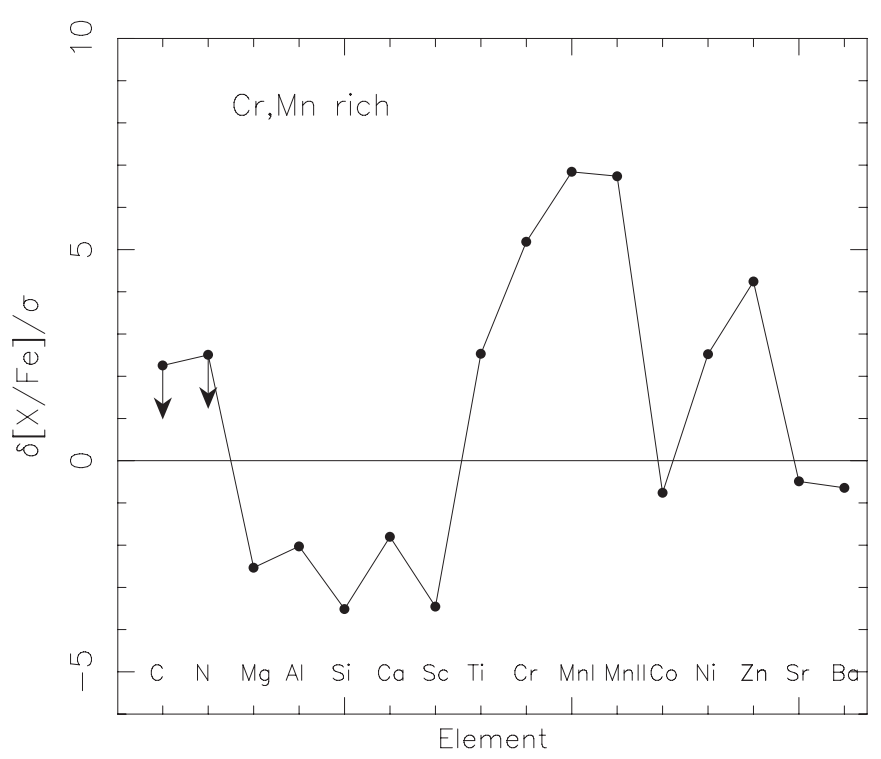

Figure 15. Same for HE2344-2800, a main sequence turnoff region star, which has abnormally high $\mathrm{Cr}$ and $\mathrm{Mn}$.

Christlieb (2005), with further refinements by Aoki et al. (2010). The key discriminants are whether or not the $s$-process elements (typically $\mathrm{La}$ and/or $\mathrm{Ba}$ ) are enhanced as well as having $[\mathrm{C} / \mathrm{Fe}]>1 \mathrm{dex}$ (denoted CEMP-s), whether both the $s$ and $r$-process (i.e., Eu) are enhanced (CEMP-rs), or whether there is no enhancement of the heavy elements beyond the Fe peak. Here we make an additional distinction between those stars with detectable $\mathrm{C}_{2}$ bands and those $\mathrm{C}$-rich stars which do not show $\mathrm{C}_{2}$ bands.

\subsection{C-Stars}

The C-rich stars that show bands of $\mathrm{C}_{2}$ (denoted in this paper as "C-stars" as an abbreviation for "classical carbon stars") are indicated here in the set of figures that display abundance ratios by a star symbol; the color of the star symbol indicates $T_{\text {eff }}$ as is true for each of the symbols used. There are $27 \mathrm{C}$ stars in our sample. Most of them are CEMP-s stars; as shown in Figure 9, only four of these C-stars do not have highly enhanced $[\mathrm{Ba} / \mathrm{Fe}]$, hence are classified as CEMP-no. These four stars span the full range in $[\mathrm{Fe} / \mathrm{H}]$ over which we have found CEMP-s stars, so within the very limited statistics of our sample there is no difference in their Fe-metallicity distribution. Two of these four stars are binaries with measured periods (see Table 2). One of these four CEMP-no stars is a dwarf with $T_{\text {eff }} \sim 6500 \mathrm{~K}$. There are two stars with very high $[\mathrm{C} / \mathrm{Fe}]$ and $[\mathrm{Ba} / \mathrm{Fe}]$ which do not show $\mathrm{C}_{2}$ bands. Both of these are quite hot with $T_{\text {eff }}>6350 \mathrm{~K}$; the $\mathrm{C}_{2}$ bands presumably are not detected due to the high $T_{\text {eff }}$. Here we classify these two stars as C-rich, but perhaps they should really be considered C-stars. One of these two is the CEMP-rs star, HE2148-1247, discussed in detail in Cohen et al. (2003).

There are more C-stars in our $0 \mathrm{Z}$ sample than one might expect if the fraction of CEMP stars at low metallicity in the Galactic halo is about $15 \%$ (estimates in the literature range from about 10 to $20 \%$ for the HES sample below -2 dex (14\% Cohen et al 2005; 9\% Frebel et al. 2006; $21 \%$ Lucatello et al. 2006). As noted in Section 2, C-stars are over-represented in our sample of candidate EMP stars from the HES as a consequence of a problem in the initial sample selection for our high resolution work; see Cohen et al. (2005). 
The CEMP-s stars are by now well understood as lowmetallicity analogs of the $\mathrm{CH}$ and $\mathrm{Ba}$ stars whose characteristics are reviewed by Wallerstein \& Knapp (1998). We believe they are binaries where we see the secondary star, while the (initially) more massive primary star has already become a low luminosity white dwarf. The third dredge up during the thermal pulses of the AGB phase of stellar evolution brings material to the surface of the primary which is enhanced in $\mathrm{C}$ and in the $s$-process elements. It is subsequently transferred to the secondary via a strong stellar wind. Our radial velocity monitoring for these CEMP-s stars (see Table 2 and the discussion in Section 4) confirms that essentially all of these stars are binaries.

The theoretical basis for this, and its application to CEMP-s stars, has been explored extensively in the literature; see, e.g., Busso et al. (1999). Recent models of nucleosynthesis in AGB stars including those of Bisterzo et al. (2011) and of Lugaro et al. (2012) apply the latest theoretical stellar evolution models varying the mass and metallicity of the AGB star, the details of the ${ }^{13} \mathrm{C}$ pocket within which neutrons can be produced, and the dilution factor (the ratio of the mass accreted by the secondary star to that of its outer convective envelope) to reproduce in detail the chemical inventory of a large number of such stars with data from the literature. Subsequent to our early suggestion of accretion induced collapse as a source of $r$-process elements to produce CEMP-rs stars outlined in Cohen et al. (2003), there have been a number of additional suggestions of how to accomplish this; see Bisterzo et al. (2011) for a recent discussion.

One other point of interest is that the $[\mathrm{Sr} / \mathrm{Fe}]$ ratio rises only modestly in CEMP-s stars as compared to the $[\mathrm{Ba} / \mathrm{Fe}]$ ratio, which sometimes rises to very high levels, enhancements approaching a factor of 100 above the solar value. This suggests that the $s$-process production of $\mathrm{Ba}$ is much more efficient than that of Sr. However, there is no enhancement of either of these elements above the solar value at $[\mathrm{Fe} / \mathrm{H}]<-3.3 \mathrm{dex}$.

\subsection{The Most Metal-poor C-rich Stars}

HE1150-0428 is the most metal-poor genuine C-star in our sample, with $[\mathrm{Fe} / \mathrm{H}]-3.2$ dex. Below this metallicity, there are no stars with detected $\mathrm{C}_{2}$ bands, even though there are still a few C-rich stars. Of the 23 stars in our sample with lower $[\mathrm{Fe} / \mathrm{H}], 4(17 \%)$ have $[\mathrm{C} / \mathrm{Fe}]>0.7 \mathrm{dex}$, but none of these show $\mathrm{C}_{2}$ bands. We believe that the apparent deficit of C-stars with $\mathrm{C}_{2}$ bands at the lowest metallicities is, at least in part, an artifact of our nomenclature. Three of these four C-rich stars have $T_{\text {eff }} \geqslant 5550 \mathrm{~K}$. The fourth has $[\mathrm{Fe} / \mathrm{H}]$ a factor of 5 lower than the most Fe-poor C-star. Since the strength of bands of $\mathrm{C}_{2}$ is roughly proportional to $N(C)^{2}$, while those of $\mathrm{CH}$ depend only on the first power of $N(C)$, we ascribe the disappearance of genuine $\mathrm{C}$-stars with detectable bands of $\mathrm{C}_{2}$ (see Figure 5) to the lower $\mathrm{Fe}$ abundance (taking $\mathrm{Fe}$ as a proxy for $\mathrm{C}$ ) and to the (by chance) tendency toward somewhat higher $T_{\text {eff }}$ to explain the abrupt disappearance of C-stars at the lowest metallicities. There may also be a shift toward higher $\mathrm{O}$ abundance, i.e., lower $\mathrm{C} / \mathrm{O}$ ratios, but evidence to support this is weak at present.

In terms of other abundance ratios, roughly half the C-rich stars below -3.3 dex have high $[\mathrm{Na} / \mathrm{Fe}]$ and $[\mathrm{Al} / \mathrm{Fe}]$, but comparably high values can be found in somewhat higher Fe-metallicity C-stars. The only genuine outliers beyond any expectation are the very high $[\mathrm{Mg} / \mathrm{Fe}]$ ratios found for HE1012-1540 and HE2323-0256 (aka CS22949-037; see Figure 5); CS22948-034 (Aoki et al. 2002) is similar to these two CEMP outliers. This small family of C-rich stars is dis- cussed in 8.3; see Figure 14. All their other abundance ratios are within or close to the range spanned by the somewhat higher Fe-metallicity C-stars for the species $\mathrm{Mg}$ to $\mathrm{Ni}$.

Three of these four stars have sub-solar $[\mathrm{Ba} / \mathrm{Fe}]$, while the fourth has $[\mathrm{Ba} / \mathrm{Fe}] \sim 0.3 \mathrm{dex}$, so none of these would be considered CEMP-s stars using a conventional definition of highly super-solar $[\mathrm{Ba} / \mathrm{Fe}]$. However, at these low metallicities, the $\mathrm{Ba} / \mathrm{Fe}$ ratio is falling rapidly as $[\mathrm{Fe} / \mathrm{H}]$ decreases, and these four stars do lie at the upper end of the sample in $[\mathrm{Ba} / \mathrm{Fe}]$ for their low Fe-metallicities. Unfortunately, none of them has a detection of $\mathrm{Eu}$, so we cannot distinguish between $r$ and $s$-process production of the $\mathrm{Ba}$.

We suggest that the $\mathrm{C}$-rich stars at the lowest Fe-metallicities we have found in our sample from the HES are the remnants of binary mass transfer systems, but that the evolution and nucleosynthesis involved are somewhat different from the picture at higher (but still very low) Fe metallicity. We make this suggestion in part because these stars comprise a fraction of the total in the sample at such low metallicity which is similar to that of the known binaries (i.e., the CEMP-s stars) at only slightly higher metallicity. Presumably there are binary systems among the most extreme EMP stars in our sample, and some of them would engage in mass transfer, and perhaps be seen today as the C-rich EMP stars below $[\mathrm{Fe} / \mathrm{H}]-3.2$ dex. If this scenario is valid, then the total amount of $\mathrm{C}$ transferred in these extreme cases must be lower, otherwise we would see a detectable rise in $[\mathrm{C} / \mathrm{Fe}]$ as $[\mathrm{Fe} / \mathrm{H}]$ decreases below $-3 \mathrm{dex}$, which we do not. This would require either less $\mathrm{C}$-enriched material being transferred from the donor star in very low metallicity binary systems or less C-enhancement of that material.

Another possibility that we have offered earlier is that these stars are the EMP equivalent of the R-type carbon stars, which are not binaries. However, these stars are somewhat hotter than the conventional R-type carbon stars.

A number of theoretical papers simulating low metallicity AGB models (Lau et al. 2007; Ventura \& d'Antona 2009) comment that AGB stars of lower metallicity are expected to reach a higher maximum temperature at the base of the convective envelope. Thus the lowest $Z$ models are expected to undergo a more advanced nuclear processing. Perhaps the reaction ${ }^{12} \mathrm{C}+\alpha \rightarrow{ }^{16} \mathrm{O}$ might keep $\epsilon(\mathrm{C}) / \epsilon(\mathrm{O})<1$ in the accreted material, thus avoiding the formation of $\mathrm{C}_{2}$. With regard to the absence of $s$-process enhanced $\mathrm{C}$-rich stars below $[\mathrm{Fe} / \mathrm{H}]-3.2$ dex, we first note that $[\mathrm{Ba} / \mathrm{Fe}]$ is dropping rapidly at the lowest metallicities in the entire stellar population; see Figure 9. It is difficult to make a lot of Ba when there are fewer and fewer Fe seeds to which neutrons can be added; one tends to make lead instead. This has been known for quite a while; Figure 12 of Busso et al. (1999) indicates the shift from the production of $\mathrm{Ba}$ to $\mathrm{Pb}$ among the most metal-poor AGB stars they modeled, all of which are more Fe-rich than the stars we are considering here. Furthermore, Lau et al. (2007) point out that zero metallicity stars avoid the third dredge up and hence do not produce any $s$-process elements at all. So the absence of $s$-process material in the most EMP C-rich stars should not be a surprise.

The strongest evidence against our hypothesis is that the radial monitoring programs, fragmentary as they are, since most of these stars have been discovered quite recently, do not support the suggestion that these stars are binaries, as is shown in Table 2. However, the models of Lau et al. (2007) suggest that zero metallicity massive AGB stars, even with mass as low as $5 M_{\odot}$, end their evolution as SN1.5 after/during the AGB stage as their 


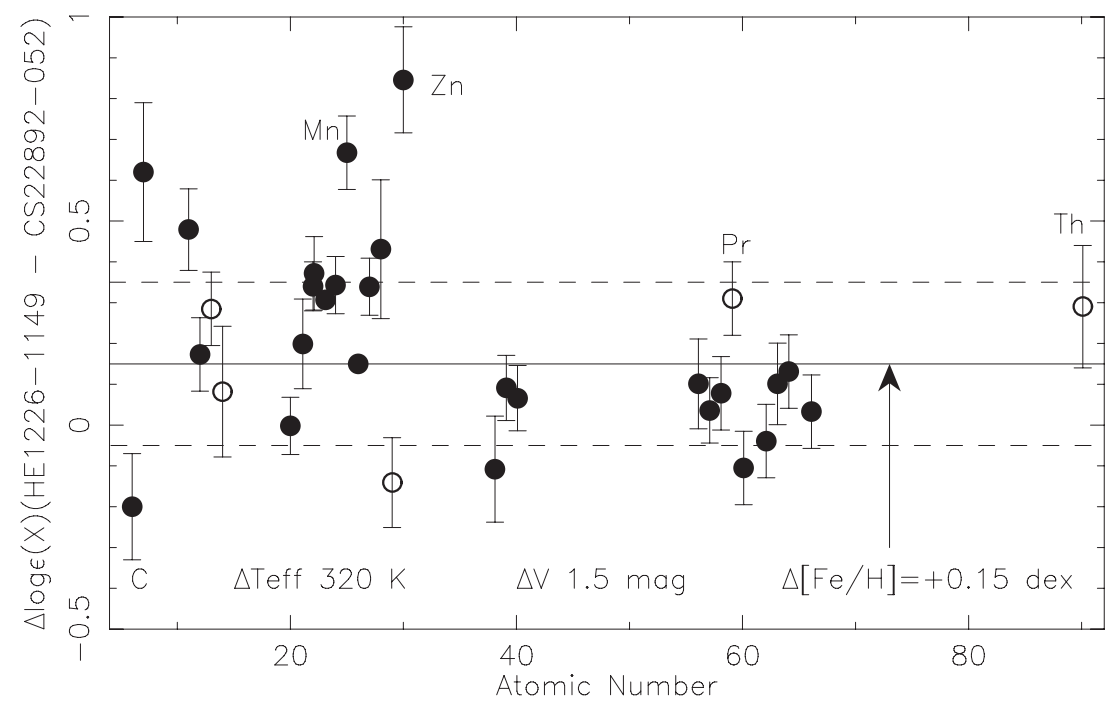

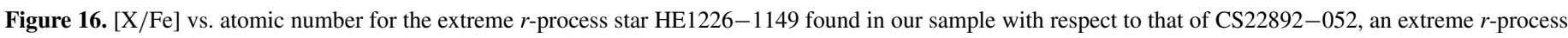

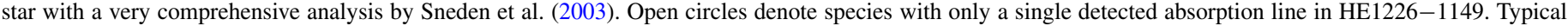

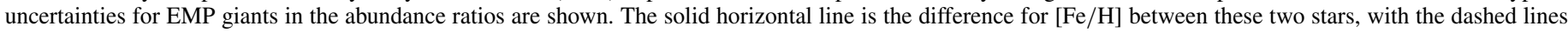
representing differences \pm 0.2 dex above or below this value.

degenerate cores are able to grow to the Chandrasekhar limit before the star loses its envelope. The envelope loss is delayed due to the weaker stellar winds expected in the lowest metallicity stars. Hence the primary explodes before it can become a white dwarf. In this case, the secondary which we now see might get a velocity kick and be ejected from the system, and that may be why the apparent fraction of binaries among these C-rich stars as viewed at the present time is not as high as in the somewhat higher metallicity CEMP-s stars.

Norris et al. (2013b) on the other hand suggest that the C-rich stars with Fe-metallicity below -3.1 dex represent a different mode of star formation in a very low metallicity environment than do the C-normal stars of similar Fe-metallicity. They ascribe the star formation for the $\mathrm{C}$-rich population to cooling via fine structure transitions of $\mathrm{C}$ II and $\mathrm{O}$ I, while a second unspecified but different cooling mechanism led to the formation of the C-normal stars.

A careful study of the crucial elements, including CNO, in the most metal-poor C-rich stars, as well as further radial velocity monitoring and theoretical modeling, and of course increasing still further the sample size of stars at these extremely low metallicities, will help to unravel this puzzle.

\section{0. $R$-PROCESS ENHANCED STARS}

A small number of stars with highly enhanced europium (i.e., with $[\mathrm{Eu} / \mathrm{Fe}]>1 \mathrm{dex}$ ) have been discovered among the very metal-poor stars, as well as a larger number with more modest $r$-process enhancements. Among the best studied of these is CS22892-052, which was analyzed in great detail by Sneden et al. (2003). Even initially it was apparent that the heavy elements beyond the Fe-peak in this and similar highly Eu-enhanced stars follow the canonical $r$-process distribution (scaled by a constant value for a given star). Sneden et al. (2009) refine their abundances for five $r$-process rich stars with improved atomic data and spectra to demonstrate how extremely closely the distributions of these heavy neutron capture elements in these stars follow the solar $r$-process distribution with $\sim 15$ rare earth elements detected, while BD+17 3248 now has 32 heavy neutron capture elements with spectroscopic abundances
(Roederer et al. 2010). Aoki et al. (2010) recently discovered the most extreme such star known to date, with $[\mathrm{Eu} / \mathrm{Fe}]+1.9$ dex, also unusual as it is a main-sequence star rather than a giant; this star is not $\mathrm{C}$-enhanced.

We have found one new extreme $r$-process enhanced star in our sample, HE1226-1149, with $[\mathrm{Eu} / \mathrm{Fe}]+1.6 \mathrm{dex}$ and $[\mathrm{Eu} / \mathrm{Ba}]+0.65$ dex; see Figure 9. This star is not C-rich. As is typical for such stars, it has $[\mathrm{Fe} / \mathrm{H}]-2.9$ dex and is a giant with $T_{\text {eff }}=5120 \mathrm{~K}$. The abundance ratios $[\mathrm{X} / \mathrm{Fe}]$ for this star for 13 heavy-neutron capture elements are compared with those of CS22892-052 taken from Sneden et al. (2003) in Figure 16. HE1226-1149, has approximately the same absolute abundances of these elements as does CS22892-052, but a slightly higher (by only $0.15 \mathrm{dex}$ ) Fe abundance. To within the observational errors the abundance ratios of the heavy neutroncapture elements follow the standard $r$-process abundance distribution. HE0011-0035 and HE2244-2116, both with $[\mathrm{Eu} / \mathrm{Fe}] \sim+0.65 \mathrm{dex}$, are less extreme $r$-process enhanced stars.

It is interesting that extremely $r$-process enhanced stars seem to occur only within a small range in $[\mathrm{Fe} / \mathrm{H}]$ near -3.0 dex; see, e.g., Barklem et al. (2005). Presumably they result from an input from a single source (i.e., a single $\mathrm{SN}$ of the appropriate type), which adds a fixed (to first order) $\Delta \epsilon(\mathrm{Eu})$. At higher $[\mathrm{Fe} / \mathrm{H}]$, this will not be enough to significantly raise the $[\mathrm{Eu} / \mathrm{Fe}]$ ratio. Their absence at lower $[\mathrm{Fe} / \mathrm{H}]$ may be related to the rapid decline of $[\mathrm{Ba} / \mathrm{Fe}]$ at the lowest metallicities shown in Figure 9.

The detections of $\mathrm{Eu}$ are quite uncertain among the extreme C-stars as there are no strong lines of Eu II redder than $4130 \AA$, and the spectra of such $\mathrm{C}$-rich stars are very crowded with molecular features of $\mathrm{CH}$ and $\mathrm{CN}$ at the relevant wavelengths. We rechecked the detections of $\mathrm{Eu}$ in all such stars again in 2012 December, and modified the most uncertain of them to upper limits, but there still may be some cases where molecular features are being mistaken for Eu lines. However, in most cases, even in the nine C-stars for which we still claim a detection of $\mathrm{Eu}$, multiple lines of Eu have been detected which each give consistent abundances for this element. For these nine C-stars, the median [Eu/Ba] is -0.8 dex, a typical $s$-process ratio, showing the significant difference in this key abundance ratio between the $r$-process seen in HE1226-1149, where 


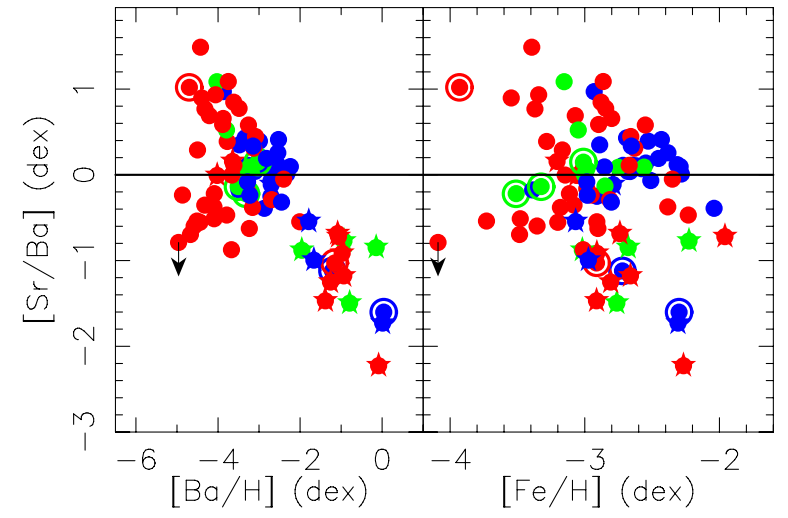

Figure 17. $[\mathrm{Sr} / \mathrm{Ba}]$ is shown as a function of $[\mathrm{Ba} / \mathrm{H}]$ (left panel) and of $[\mathrm{Fe} / \mathrm{H}]$ (right panel). Upper limits are not shown unless $[\mathrm{Ba} / \mathrm{H}]<-4.5$ dex. Symbols are as in Figure 3. The error bars in each panel of this figure are comparable in size to the points.

(A color version of this figure is available in the online journal.)

[Eu/Ba] is $+0.6 \mathrm{dex}$, and the $s$-process of neutron capture formation of the heavy elements seen in the C-stars.

At the lowest metallicities probed here, even in stars which are not C-rich, it is quite difficult to detect Eu unless it is highly enhanced. The predicted line strengths for $[\mathrm{Eu} / \mathrm{Fe}]+0.5 \mathrm{dex}$ at $T_{\text {eff }} \sim 5000 \mathrm{~K}$ and $[\mathrm{Fe} / \mathrm{H}]-3.2$ dex are below $10 \mathrm{~m} \AA$ for even the strongest Eu II lines in the optical wavelength regime reaching down to $3800 \AA$, making it difficult to reliably detect any Eu lines. Since our sample stars are faint, there are many upper limits for their europium abundances, and, as shown in Figure 9, there are no solid detections of Eu for any star in our sample below $[\mathrm{Fe} / \mathrm{H}]=-3.3$ dex. Compounding the difficulty of detecting Eu in EMP stars is the general trend of an increasing deficiency in the heavy neutron capture elements toward lower Fe metallicity best shown in Figure 9 for $[\mathrm{Ba} / \mathrm{Fe}]$.

With these caveats in mind, we compare the frequency of extreme $r$-process enhanced stars found by the HERES project (Barklem et al. 2005) with that found here. In both cases, hot dwarfs are excluded as almost all extreme $r$-process enhanced stars known have $T_{\text {eff }}$ near $5100 \mathrm{~K}$, and stars with strong $\mathrm{CH}$ are also excluded. The agreement for the fraction of extremely $r$-process enhanced stars, given the very small numbers involved, is reasonable. Adopting the definition of Barklem et al. (2005) for moderately $r$-process enhanced stars of $0.3<[\mathrm{Eu} / \mathrm{Fe}]<1.0$ dex, with the same exclusions, we find that $8 \%$ of our $0 \mathrm{Z}$ sample is moderately $r$-process enhanced. This is somewhat smaller than the fraction found by Barklem et al. (2005) of $14 \%$. The lower mean $[\mathrm{Fe} / \mathrm{H}]$ values in our $0 \mathrm{Z}$ sample lead to a higher fraction of stars with only upper limits for their Eu abundance, and this probably gives rise to the lower fraction of moderately enhanced $r$-process stars in our sample as compared with that of the HERES project.

\subsection{Heavy Neutron Capture Elements in Normal EMP Stars}

With the exception of the C-stars, the ratios among the heavy neutron capture elements among EMP stars appear to follow the $r$-process rather than the $s$-process ratios, when such a distinction is possible. However, given the many upper limits to Eu, this is not easy to verify for most of our sample of EMP stars from the HES for the reasons discussed in Section 10. The relative abundances $[\mathrm{X} / \mathrm{Fe}]$ of the heavy neutron capture elements show both a tremendous variation in their overall depletion with respect to $\mathrm{Fe}$, as is illustrated in Figure 9, and

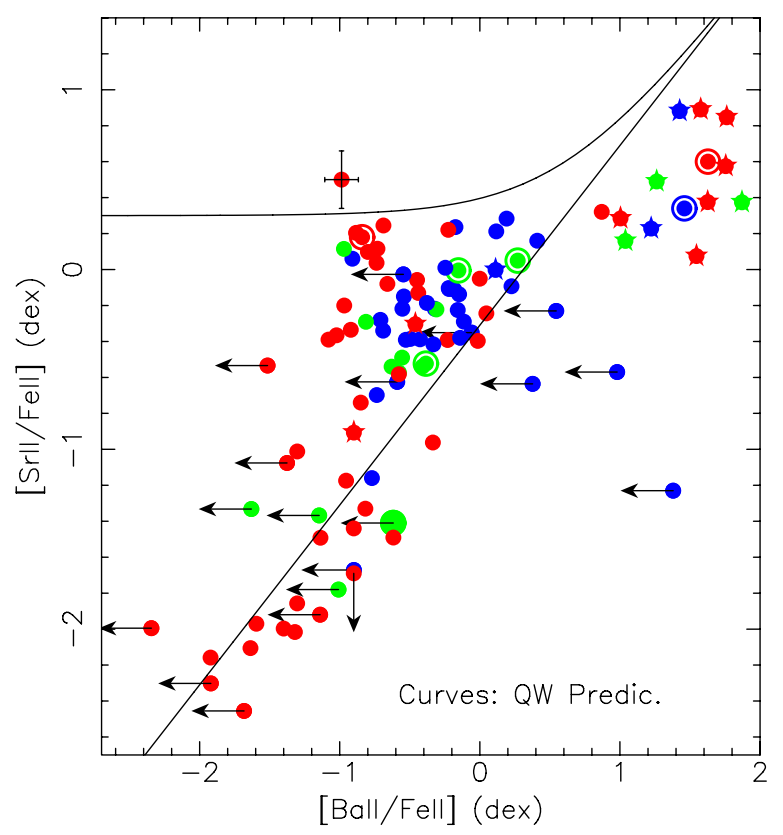

Figure 18. Prediction by Qian \& Wasserburg (2008) of the allowed region (the area between the two curves) of $[\mathrm{Sr} / \mathrm{Fe}]$ as a function of $[\mathrm{Ba} / \mathrm{Fe}]$ is compared with our derived abundance ratios. Symbols are as in Figure 3. A typical error bar is shown for a single star.

(A color version of this figure is available in the online journal.)

also variations in the ratios of the first peak (i.e., $\mathrm{Sr}$ ) versus those in the second peak (i.e., Ba). The need for an additional channel to reach the unexpectedly high $[\mathrm{Sr} / \mathrm{Ba}]$ ratios seen in some EMP stars has been widely discussed in the literature (see, e.g., McWilliam et al. 1995a; Travaglio et al. 2004a; Bisterzo et al. 2011).

Figure 17 shows $[\mathrm{Sr} / \mathrm{Ba}]$ both as a function of $[\mathrm{Ba} / \mathrm{H}]$ and also of $[\mathrm{Fe} / \mathrm{H}]$. Stars with only upper limits are not shown except for the most metal-poor star in our sample, with an upper limit for $\mathrm{Sr}$ and a very marginal detection of $\mathrm{Ba}$. As contrasted with the large range and chaos seen in plots of $[\mathrm{Sr} / \mathrm{Fe}]$ and $[\mathrm{Ba} / \mathrm{Fe}]$ versus $[\mathrm{Fe} / \mathrm{H}]$ (see Figure 9) or the somewhat less chaotic relation of $[\mathrm{Sr} / \mathrm{Ba}]$ versus $[\mathrm{Fe} / \mathrm{H}]$, the left panel of our Figure $17^{14}$ shows a much clearer relation such that the lowest $\mathrm{Ba}$ stars seem to have the largest enhancement of $\mathrm{Sr}$ relative to $\mathrm{Ba}$. This implies that the production of the heavy neutron capture elements is occurring with well defined relative ratios which are a function of the Ba abundance, but these processes are decoupled in their efficiency from those that produce the Fe-peak elements. One can also view this in terms of mixing rather than production, i.e., mixing into the almost pristine gas of the proto-Galactic halo of the ejected neutron-capture process material. Perhaps this is very localized compared to that for the Fe-peak elements. Perhaps the neutron-capture processes require a special rare type of SNII, so that stochastic local mixing becomes important.

Qian \& Wasserburg (2008) have presented a phenomenological theory to explain the abundances of the heavy-neutron capture elements by mixing ejecta of several different types of SNII that might occur in the early formation of the Galaxy. They predict allowed regions in the plane $[\mathrm{Sr} / \mathrm{Fe}]$ as a function of $[\mathrm{Ba} / \mathrm{Fe}]$, which are illustrated as lines in Figure 18, with our data superposed. The agreement is quite good in that essentially

\footnotetext{
14 A figure similar to the left panel of our Figure 17 based on results from the
} First Stars project can be found in Francois et al. (2008, their Figure 15). 
Table 16

Abundance Ratios Grouped by Distance

\begin{tabular}{|c|c|c|c|c|c|c|c|c|c|}
\hline \multirow[t]{2}{*}{ Species } & \multicolumn{3}{|c|}{ Close $^{a}$} & \multicolumn{3}{|c|}{ Inner Halo $^{b}$} & \multicolumn{3}{|c|}{ Outer Haloc } \\
\hline & $\begin{array}{c}\langle[\mathrm{X} / \mathrm{H}]\rangle \\
(\mathrm{dex})\end{array}$ & $\begin{array}{c}N^{\mathrm{d}} \\
(\mathrm{dex})\end{array}$ & $\sigma$ & $\begin{array}{c}\langle[\mathrm{X} / \mathrm{H}]\rangle \\
(\mathrm{dex})\end{array}$ & $\begin{array}{c}N^{\mathrm{d}} \\
(\mathrm{dex})\end{array}$ & $\sigma$ & $\begin{array}{c}\langle[\mathrm{X} / \mathrm{H}]\rangle \\
(\mathrm{dex})\end{array}$ & $\begin{array}{c}N^{\mathrm{d}} \\
(\mathrm{dex})\end{array}$ & $\sigma$ \\
\hline$[\mathrm{Fe} / \mathrm{H}]$ & -2.86 & 54 & 0.37 & -2.88 & 58 & 0.51 & -3.02 & 10 & 0.40 \\
\hline$[\mathrm{Na} / \mathrm{Fe}]$ & 0.16 & 21 & 0.65 & 0.18 & 49 & 0.52 & 0.28 & 8 & 0.38 \\
\hline$[\mathrm{Mg} / \mathrm{Fe}]$ & 0.39 & 54 & 0.24 & 0.39 & 59 & 0.25 & 0.49 & 10 & 0.16 \\
\hline$[\mathrm{Al} / \mathrm{Fe}]$ & -0.12 & 53 & 0.25 & -0.18 & 47 & 0.27 & -0.06 & 10 & 0.30 \\
\hline$[\mathrm{Si} / \mathrm{Fe}]^{\mathrm{e}}$ & 0.09 & 44 & 0.26 & 0.42 & 47 & 0.41 & 0.41 & 9 & 0.31 \\
\hline$[\mathrm{Ca} / \mathrm{Fe}]$ & 0.30 & 54 & 0.13 & 0.22 & 56 & 0.22 & 0.25 & 10 & 0.25 \\
\hline$[\mathrm{Sc}$ II $/ \mathrm{Fe}$ II] & 0.20 & 47 & 0.27 & 0.13 & 46 & 0.23 & 0.09 & 10 & 0.13 \\
\hline [Ti II/Fe II] & 0.28 & 53 & 0.14 & 0.26 & 59 & 0.22 & 0.10 & 10 & 0.18 \\
\hline$[\mathrm{Cr} / \mathrm{Fe}]$ & -0.26 & 50 & 0.12 & -0.36 & 59 & 0.19 & -0.41 & 10 & 0.13 \\
\hline$[\mathrm{Mn} / \mathrm{Fe}]$ & -0.57 & 49 & 0.19 & -0.63 & 51 & 0.34 & -0.71 & 10 & 0.25 \\
\hline$[\mathrm{Co} / \mathrm{Fe}]^{\mathrm{f}}$ & 0.39 & 38 & 0.22 & 0.36 & 41 & 0.30 & 0.17 & 9 & 0.23 \\
\hline$[\mathrm{Ni} / \mathrm{Fe}]$ & -0.06 & 38 & 0.14 & -0.08 & 42 & 0.28 & -0.10 & 8 & 0.27 \\
\hline$\left[\mathrm{Sr} \text { II } / \mathrm{Fe} \mathrm{II}^{\mathrm{f}}\right]^{\mathrm{f}, \mathrm{g}}$ & -0.45 & 36 & 0.51 & -0.71 & 37 & 0.79 & -1.13 & 9 & 1.10 \\
\hline$[\mathrm{Ba} \text { II } / \mathrm{Fe} \text { II }]^{\mathrm{f}, \mathrm{g}}$ & -0.31 & 36 & 0.58 & -0.78 & 37 & 0.68 & -1.10 & 9 & 0.66 \\
\hline
\end{tabular}

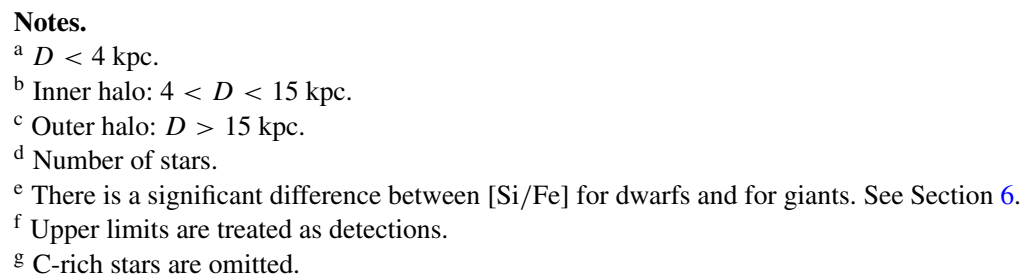

all of the stars in our sample (ignoring the C-stars) lie within the area designated as allowed.

\section{DISTANCE EFFECTS}

We have looked for a dependence of chemical inventory on distance within our in-situ HES EMP sample. We first calculate the absolute luminosity for each star from its observed $V$ mag and adopted reddening. We then interpolate between the $\alpha$ enhanced isochrones of $\mathrm{Yi}$ et al. (2003) for $[\mathrm{Fe} / \mathrm{H}]-1.2,-2.2$, and -3.2 dex at a fixed age of $12 \mathrm{Gyr}$ to find the predicted $M_{V}$ at the $T_{\text {eff }}$ we adopt given the $[\mathrm{Fe} / \mathrm{H}]$ we have deduced for the star. We assume the star is at or more luminous than the main sequence turnoff. The distances thus derived for EMP stars from the HES range from $0.5 \mathrm{kpc}$ for the nearest dwarfs ${ }^{15}$ to $34 \mathrm{kpc}$, with 10 EMP giants lying beyond $15 \mathrm{kpc}$. We divide our $0 \mathrm{Z}$ sample into three groups, close ( $D<4 \mathrm{kpc}, 54$ stars), inner halo (4<D<15 kpc, 59 stars), and outer halo $(D>15 \mathrm{kpc}$, 10 stars). We looked for a relationship between $[\mathrm{Fe} / \mathrm{H}]$ and $D$, but, as indicated in Table 16, did not find anything. The mean and dispersion for the 10 outer halo giants observed in situ is $[\mathrm{Fe} / \mathrm{H}]=-3.02$ dex with $\sigma 0.39 \mathrm{dex}$; the median is -2.90 dex. For the 54 inner halo stars we find a median of -2.88 dex, essentially identical. Note that neither of the two peculiar CEMP-no extreme EMP stars with highly enhanced $\mathrm{Na}$ and $\mathrm{Mg}$ discussed in Section 9 belongs to the outer halo group.

Recently, the huge and homogeneous data archive of SDSS and SEGUE spectra has been used to search for metallicity gradients in the halo, but the results of Carollo et al. (2007; see also de Jong et al. 2010) are in conflict with those of Sesar et al. (2011), who used the CFHT Legacy Survey. Sesar et al. (2011) find the median metallicity to be independent of distance out to

\footnotetext{
15 Our sample includes two bright previously known very metal-poor dwarfs
} which are both closer than $0.5 \mathrm{kpc}$. at least $25 \mathrm{kpc}$. The most recent SDSS results from Schlaufman et al. (2012) find no gradient in the mean $[\mathrm{Fe} / \mathrm{H}]$ in the halo out to at least $17 \mathrm{kpc}$. Although ours is an in-situ sample, our data is not ideal for this purpose, as we have not taken into account the potential effect of the selection function for EMP stars within the HES, which strongly suppresses the higher metallicity part of the distribution (Schörck et al. 2009), and thus tends to make the medians for the two distance ranges approach each other. Furthermore, due to lack of proper motions, we do not know the 3D orbits of most of our sample stars, so there may be considerable blurring of any distinction between the inner and outer halo.

We have also looked for distance effects on the abundance ratios. The ratios $[\mathrm{Mg} / \mathrm{Fe}]$ and $[\mathrm{Ca} / \mathrm{Fe}]$ are of particular interest as there have been suggestions in the literature of a decline in $\alpha$ enhancement between the inner and outer halo, which is shown most clearly in the SDSS-based recent survey of the smooth halo by Schlaufman et al. (2012, see their Figure 4). However, we have not found any systematic difference with distance for most of the abundance ratios with suitable data from our in situ halo field star sample. In particular, as shown in Figure 19, the mean $[\mathrm{Ca} / \mathrm{Fe}]$ ratio appears to be independent of distance. However, the lack of a strong gradient in $\alpha / \mathrm{Fe}$ with distance in our sample may result at least in part from sampling issues; our data are confined to the most metal-poor population at all distances.

The only potential distance effect that we noticed in checking all the abundance ratios for those species for which we had enough data was for $[\mathrm{Sr} / \mathrm{Fe}]$ and for $[\mathrm{Ba} / \mathrm{Fe}]$. As shown in Figure 20 and in Table 16, there is a hint that the outer halo stars have systematically ratios of $[\mathrm{Sr} / \mathrm{Fe}]$ systematically lower by $\sim 0.4$ dex at a given $[\mathrm{Fe} / \mathrm{H}]$ than do inner halo stars; $[\mathrm{Ba} / \mathrm{Fe}]$ appears to display the same trend with distance, but is complicated by the many upper limits for $\mathrm{Ba}$ abundances among our sample stars. Statistical measures of abundance ratios as a function of distance are given in Table 16. Ignoring [Si/Fe], 


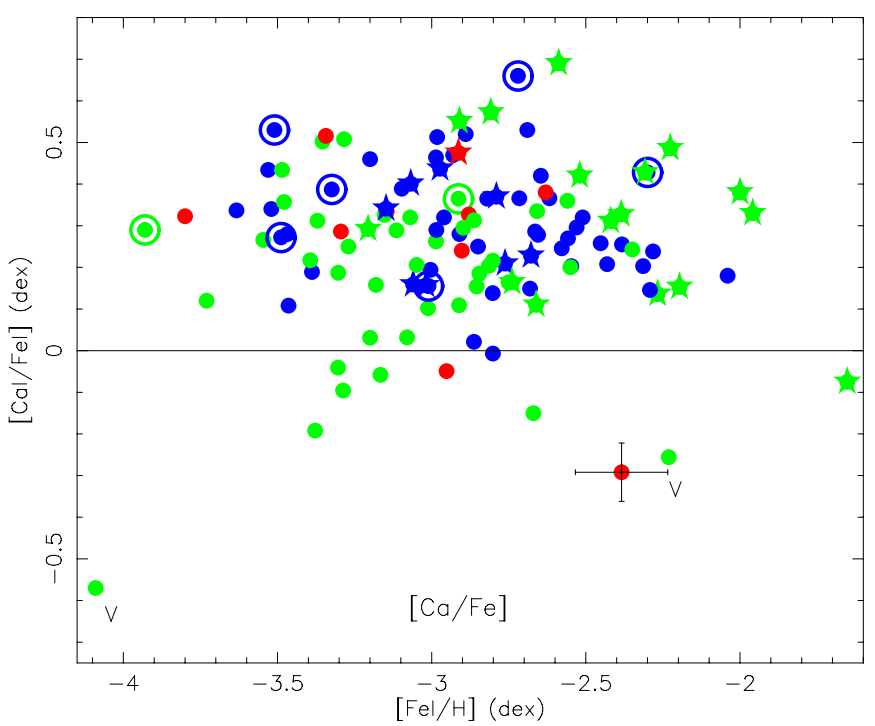

Figure 19. As in Figure 6, but with colors denoting distance; blue: close, green: inner halo, red: outer halo. A typical error bar is shown for a single star.

(A color version of this figure is available in the online journal.)

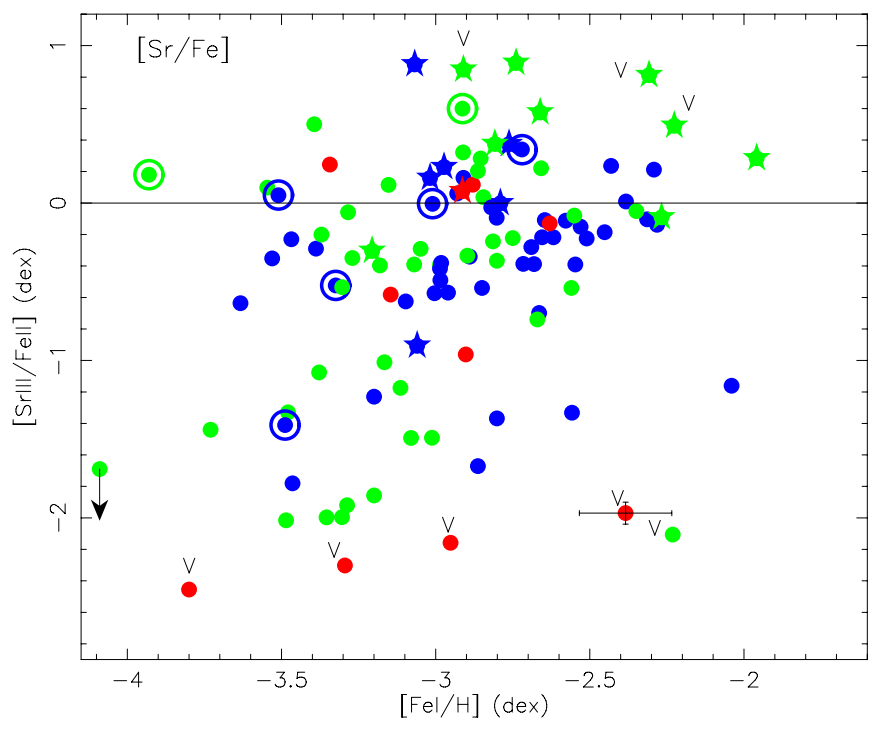

Figure 20. Same for the ratio $[\mathrm{Sr} / \mathrm{Fe}]$. The ratio $[\mathrm{Sr} / \mathrm{Fe}]$ appears to be lower among the most distant stars than among the nearer stars of the same $[\mathrm{Fe} / \mathrm{H}]$.

(A color version of this figure is available in the online journal.)

where there is an obvious analysis problem between dwarfs and giants (see Section 6), the total range in the mean from $D<4 \mathrm{kpc}$ to $D>15 \mathrm{kpc}$ with in situ measurements is large only for $[\mathrm{Sr}$ II/Fe II] and for $[\mathrm{Ba}$ II/Fe II]; most of the other abundance ratios show maximum differences in the mean abundance ratio between the three distance bins of 0.15 dex or less.

There have been a large number of studies of halo stars passing through the solar neighborhood; see, e.g., Stephens \& Boesgaard (2002), Fulbright (2002), Gratton et al. (2003), Roederer (2009), Nissen \& Schuster (2010, 2011). These have the advantage that the stars, being much closer than our in situ halo sample, are brighter, and often have parallaxes, so that a Galactic orbit can be computed, allowing a clean kinematic separation between inner and outer halo stars. Overall, these studies find small systematic differences in abundance ratios between the mean $[\mathrm{Mg} / \mathrm{Fe}]$ at a level of 0.1 to $0.2 \mathrm{dex}$, with

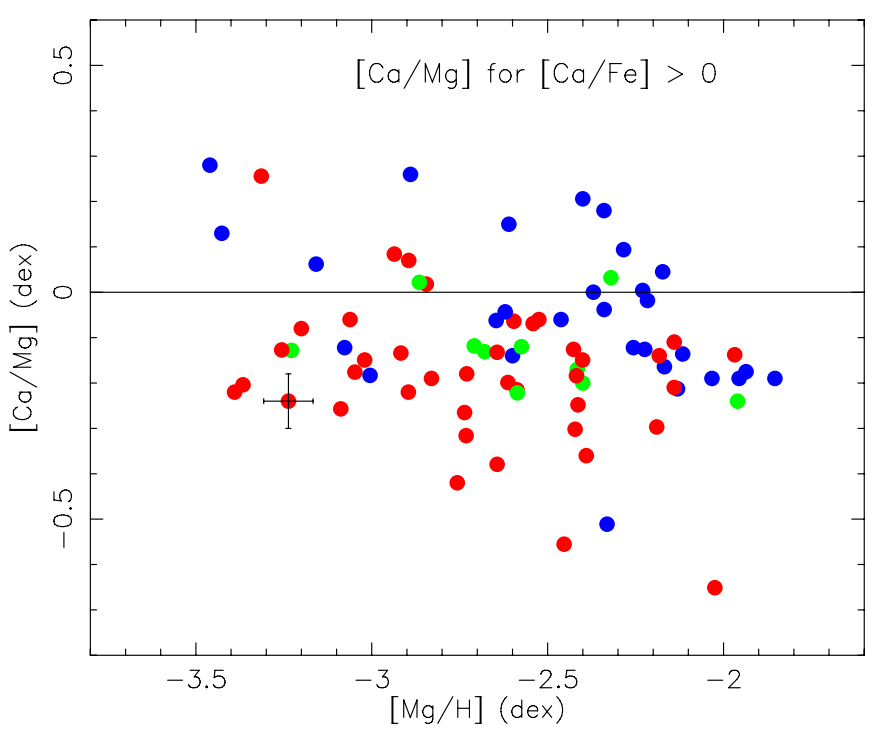

Figure 21. $[\mathrm{Ca} / \mathrm{Mg}]$ is shown as a function of $[\mathrm{Mg} / \mathrm{H}]$ for the $\mathrm{C}$-normal stars with $[\mathrm{Ca} / \mathrm{Fe}]>0$ in our $0 \mathrm{Z}$ sample. Symbols are as in Figure 3. Notes regarding checks of outliers are as in Figure 5. A typical error bar is shown for a single star.

(A color version of this figure is available in the online journal.)

the outer halo stars having lower $\alpha / \mathrm{Fe}$. Smaller such trends are sometimes claimed for $\mathrm{Si}, \mathrm{Ca}, \mathrm{Ti}$, and $\mathrm{Ni}$, with even more subtle and smaller differences claimed by Nissen \& Schuster (Nissen \& Schuster 2010, 2011) in the behavior of [Zn,Y, Ba/Fe]. Many of the low $\alpha$ stars have retrograde orbits, have ages 2 Gyr younger than those of the normal- $\alpha$ stars (Schuster et al. 2012), and have $R_{\text {apo }}>20 \mathrm{kpc}$. As indicated above, our sample, which lacks kinematic data, is not ideal for this purpose. Furthermore, we cannot hope to achieve the precision abundances that can be obtained for the much brighter solar neighborhood stars selected to have very similar stellar parameters; a 0.1 dex difference in abundance ratio is too small to be detectable in our sample of EMP stars given the uncertainties in our data.

\section{COMMENTS ON NUCLEOSYNTHESIS AND RELATED ISSUES}

Models of nucleosynthesis in SNIa and of SNII physics including ejection mechanisms, fallback, and mixing within the ejecta (Kobayashi et al. 2006; Tominaga et al. 2007; Heger \& Woosley 2010) have been developed that can successfully explain the chemical inventory of (most) EMP Galactic halo stars. Here we highlight the outliers from the general trends established by the many recent studies of EMP halo field stars, the major one of which (in addition to our own $0 \mathrm{Z}$ project), is the First Stars project (Cayrel et al. 2004).

Before doing so, we consider evidence from the $0 \mathrm{Z}$ project regarding the possible variations in SNII progenitor mass. The ratio $\mathrm{Ca} / \mathrm{Mg}$ varies significantly with the progenitor mass of SNII; see, e.g., the grid of SNII yields by Heger \& Woosley (2010). Figure 21 displays that ratio for our $0 \mathrm{Z}$ sample as a function of $[\mathrm{Mg} / \mathrm{H}]$, ignoring the C-rich stars and the largest group of outliers, the low-Ca family. It is apparent that there are small systematic offsets between the mean relation for the giants, subgiants, and main sequence turnoff region stars, with the giants having higher $[\mathrm{Mg} / \mathrm{Ca}]$ than the subgiants, and those higher than the dwarfs. This is presumably a result of the small dependence of non-LTE corrections for $\mathrm{Mg}$ and for $\mathrm{Ca}$ on stellar parameters discussed in Section 6.1. The nature of these offsets 
and their magnitude are close to those predicted by combining the calculations of Andrievsky et al. (2010) and Spite et al. (2012) for non-LTE in giants versus dwarfs for $\mathrm{Mg}$ and for $\mathrm{Ca}$ respectively.

The most interesting point of Figure 21 is that there is only a small change in the ratio $[\mathrm{Ca} / \mathrm{Mg}]$ with increasing $\mathrm{Mg}$ abundance, with a slope of approximately $-0.1 \mathrm{dex} / \mathrm{dex}$ for giants. A similar figure was constructed by McWilliam et al. (1995a, their Figure 24), who found a very similar slope for their sample of giants. This strongly suggests that the mean SNII progenitor mass, and hence the IMF (at least for massive stars capable of becoming SNII) varied somewhat but not significantly over the period of time during which the relevant SNII occurred. Note that this only applies for the C-normal stars with $[\mathrm{Ca} / \mathrm{Fe}]>0$ dex.

The many C-stars found among the EMP halo field stars can be explained by binary mass transfer from an AGB companion as discussed in Section 9. Although theoretical population syntheses of C-rich star production via binary mass transfer have trouble reproducing the observed high fraction of C-rich stars quantitatively, as well as the apparent relative absence of $\mathrm{N}$-rich stars, (see, e.g., Izzard et al. 2009), our radial velocity surveys (as well as those of others, see Section 4) and corresponding binary fractions strongly support the binary mass transfer scenario.

Ignoring the C-rich stars, the major class of outliers is the low-Ca stars. We believe that these are the result of variations of SNII nucleosynthesis yields with progenitor mass and perhaps other factors. Heavier progenitors produce more $\mathrm{O}$ and $\mathrm{Mg}$ with respect to $\mathrm{Ca}$ and $\mathrm{Ti}$, with the yields of Nomoto et al. (2006) showing changes in the ratio of $\mathrm{Mg}$ to $\mathrm{Ca}$ of a factor of 18 in the ejecta with progenitor mass increasing from 13 to $30 M_{\odot}$. This change could perhaps explain the low $[\mathrm{Ca} / \mathrm{Fe}]$ family of stars if one assumes a local effect (i.e., non-global, inhomogeneous mixing) from a rare higher mass SN progenitor. Presumably stochastic effects from individual early $\mathrm{SN}$ and limited mixing in the proto-Galactic halo led to stars with unusually low $\mathrm{Ca} / \mathrm{Fe}$ being formed at a low frequency $(\sim 10 \%)$ in the young halo, and not at all (or at a much lower frequency) once the cumulative number of SNII contributing to the chemical inventory of a star becomes much larger and the halo became better mixed, as is the case for the higher-metallicity disk. An explanation in terms of contributions from prompt SNIa may also be feasible, but has trouble reproducing the detailed behavior of the $\alpha$-elements in the low-Ca EMP population.

A very peculiar halo red giant, this time with normal $\mathrm{C}$, a huge excess of $\mathrm{Ca}$ and a small deficit of $\mathrm{Mg}$ relative to its $\mathrm{Fe}$, was found by Lai et al. (2009). If the lighter $\alpha$-elements such as $\mathrm{Mg}$ were also highly enhanced, one might view this star as having a strong contribution from a PISN, but $\mathrm{Mg}$ is not enhanced at all. This star is faint and quite cool with very uncertain stellar parameters (see Lai et al. 2009 for details), and their results should be checked. If their result holds, it could have arisen from a very low-mass progenitor Type II SN. Alternatively, it could be heavily contaminated by Type Ia SN ejecta. We note that no $\mathrm{C}$-normal star in our sample has $[\mathrm{Ca} / \mathrm{Fe}]>0.55 \mathrm{dex}$.

The recent surge in detailed abundance studies of the stellar population in the dwarf satellites of the Milky Way (see, e.g., Tolstoy et al. 2009; Kirby et al. 2011) has led to new insights on how the star formation history and the influence of galactic winds affects the chemical inventory of a low luminosity galaxy. In part motivated by such results, as well as by the rapid improvement in the mass resolution of cosmological dark matter simulations, simulations of the stellar component of galaxy halos have become detailed enough to follow the assembly of the halo of a galaxy such as the Milky Way. These take into account both in situ star formation as well as accretion of stars from subhalos, and follow the impact of the accretion on the chemical inventory within the Galaxy's halo. Such techniques, strongly focused on the $\alpha / \mathrm{Fe}$ ratios, were initially developed by Bullock \& Johnston (2005), Zolotov et al. (2010), and Tissera et al. (2012) have considerably advanced the art and are much more successful in reproducing the observed mean trends in our survey, although not the outliers.

Another converging thread for the study of EMP Milky Way halo stars is the increasing quantity and quality of data on damped Lyman absorbers (DLAs) found in the spectra of high redshift QSOs. The latest study by Rafelski et al. (2012) finds that the metallicity distribution and $\alpha / F e$ ratios of $z>2$ DLAs are consistent with being drawn from the same parent population as those of Milky Way halo stars. Furthermore, Cooke et al. (2012) have found a modestly C-enhanced DLA with $[\mathrm{Fe} / \mathrm{H}] \sim-2.8$ dex. Fumagalli et al. (2011) recently broke the long-standing lower metallicity limit for high redshift gas clouds (Lyman limit systems seen in QSO spectra) by finding two with upper limits of $Z<10^{-3.8} Z_{\odot}$, which is well within the EMP Galactic halo star range we are exploring here.

\section{SUMMARY}

Our $0 \mathrm{Z}$ survey had the goals of finding more EMP stars with $[\mathrm{Fe} / \mathrm{H}]<-3.0$ dex and using them (and in particular their chemical inventories) to improve our understanding of the young Milky Way, focusing on the evolution of its heavy element content, how well the ISM in the young Galaxy was mixed, and what nucleosynthesis processes contributed. This work began in 2000 with the Keck Pilot Project (Cohen et al. 2002; Carretta et al. 2002), and with this paper it concludes. Although we have published many papers along the way describing stars of particular interest, here we have attempted an overview of the entire ensemble of 122 stars for which we have obtained high dispersion spectra at either Keck or Magellan and subsequently have carried out detailed abundance analysis for each sample star. Our sample is an in-situ probe of the Milky Way halo, and $8 \%$ of the sample is more distant than $15 \mathrm{kpc}$.

The most interesting thing we have found is that, ignoring Carbon stars, $10 \%$ of the sample has sub-solar $[\mathrm{Ca} / \mathrm{Fe}]$, a phenomenon that is virtually non-existent among solar neighborhood stars and Galactic disk stars, which are considerably closer and with higher metallicities than the $0 \mathrm{Z}$ sample, as well as among Galactic globular cluster stars. These low-Ca stars also have slightly low abundances for the other $\alpha$-elements we detect $(\mathrm{Mg}$ and $\mathrm{Si})$, as well as low abundance ratios for the heavy neutron-capture elements. There are two potential explanations for producing such an unusual chemical inventory. The first involves a more top-heavy IMF in the young Milky Way, biasing the mass of the SNII progenitors higher so that the $\mathrm{Mg} / \mathrm{Ca}$ ratio in the $\mathrm{SN}$ ejecta becomes higher (Nomoto et al. 2006), thus producing a "deficiency" of Ca. The second is a substantial contribution from prompt SNIa elevating the Fe-peak but not producing much of anything else, but this hypothesis has some problems reproducing the detailed $\alpha$-element distributions.

There are smaller groups of outliers within our sample, of which the most unusual is a group of two C-rich stars where the high $\mathrm{C}$-enhancement is carried further through to high $\mathrm{N}, \mathrm{Na}$, $\mathrm{Mg}$, and/or Al. Both of the two members of this class have $[\mathrm{Fe} / \mathrm{H}]<-3.5$ dex and presumably the lack of detectable 
$\mathrm{C}_{2}$ bands is a consequence of their very low Fe-metallicity. These stars appear "normal" for the elements we can probe between $\mathrm{Ca}$ and the Fe-peak, but then show relatively high heavy-neutron capture element abundances (e.g., [Ba/Fe] +0.27 and -0.84 dex) given their very low $[\mathrm{Fe} / \mathrm{H}]$.

Multiple epochs of high-dispersion spectroscopy are available for a substantial fraction of the sample stars. While all the higher Fe-metallicity $\mathrm{C}$-stars that show $s$-process enhancement appear to be binaries, and most of the stars with $\mathrm{C}_{2}$ bands but no $s$-process enrichment also appear to be binaries, there is no evidence to support a binary origin for these two anomalous C-rich stars. We speculate that these two stars may be remnants from mass transfer binary systems involving a very low metallicity primary which ended its evolution as SN1.5 during or after the AGB phase, such the primary was able to grow to the Chandrasekhar limit before it lost its envelope. The primary thus would explode and end its stellar evolution before becoming a white dwarf.

We have also found a new extreme $r$-process enhanced star with $[\mathrm{Eu} / \mathrm{Fe}]+1.6$ dex. As expected, for the 13 heavy neutroncapture elements we could detect, its chemical inventory follows the standard $r$-process abundance ratios.

Another surprise is the very low abundances of $\mathrm{Sr}$ and $\mathrm{Ba}$ found in a substantial number of the EMP giants; 13 stars have $[\mathrm{Sr} / \mathrm{Fe}]<-1.5$ dex and 8 lie below this value for $\mathrm{Ba}$, with a few more having upper limits slightly higher than $[\mathrm{Ba} / \mathrm{Fe}]=$ $-1.5 \mathrm{dex}$. The $\mathrm{Sr} / \mathrm{Ba}$ ratio is well behaved as a function of $[\mathrm{Ba} / \mathrm{H}]$, but not so well behaved as a function of $[\mathrm{Fe} / \mathrm{H}]$, illustrating again the decoupling of the production of the heavy neutron-capture elements from that of Fe.

The behavior of C-rich stars changes below $[\mathrm{Fe} / \mathrm{H}]-3.2 \mathrm{dex}$; no stars showing bands of $\mathrm{C}_{2}$ were seen, whereas this is common among higher Fe-metallicity stars. Some potential contributing factors were discussed, including the possibility of substantial $\mathrm{O}$ enhancement keeping $\mathrm{C} / \mathrm{O}<1$.

Overall, ignoring C-stars, approximately $15 \%$ of the sample are strong outliers in one or more elements between $\mathrm{Mg}$ and $\mathrm{Ni}$. This rises to $\sim 19 \%$ if very strong outliers for $\mathrm{Sr}$ and $\mathrm{Ba}$ are included. Ignoring the strong outliers, the dispersions around linear fits of $[\mathrm{X} / \mathrm{Fe}]$ versus $[\mathrm{Fe} / \mathrm{H}]$ for the $\mathrm{C}$-normal stars are comparable to those predicted from the observational and analysis uncertainties for $\mathrm{Si}, \mathrm{Sc}$, and $\mathrm{Ni}$. They rise to $\sim 1.7$ times the expected values for $\mathrm{Ti}, \mathrm{Cr}$, and $\mathrm{Mn}$, and to $\sim 2$ for $\mathrm{Mg}$ and $\mathrm{Ca}$, rising to $\sim 4$ for $\mathrm{Sr}$ and $\mathrm{Ba}$. The dispersion around a similar linear fit for $[\mathrm{Ca} / \mathrm{Mg}]$ versus $[\mathrm{Mg} / \mathrm{H}]$ shows a small scatter once a dependence on $T_{\text {eff }}$, presumably from non-LTE effects, is removed.

The most metal-poor star in our sample has $[\mathrm{Fe} / \mathrm{H}]-4.1$ dex. Given how arduous was the path to find it, future searches for UMP or HMP stars will require a survey where extensive screening to eliminate higher metallicity interlopers is not necessary. The initial photometry (presumably including a narrow band filter centered on the $3933 \AA$ Ca II line) or spectra must discriminate well enough to cleanly isolate such stars for detailed study, which was not the case for the HES. SDSS spectra are much more suitable, but UMP and HMP stars are so rare that it may require an "all star" survey to find them in the halo of the Milky Way. While selected dSph satellites of the Milky Way have much less advanced chemical evolution, and mean $\mathrm{Fe} / \mathrm{H}$ values for their member stars more than a factor of 10 below that of the halo of the Milky Way, their low luminosity and in many cases large distances may render them less productive than a Milky Way halo field star survey.
We are very grateful to the Palomar, Las Campanas, and Keck time allocation committees for their long-term support of this campaign during the initial phase of moderate resolution spectroscopy which began in 2000 and ended in 2006 as well as the subsequent high resolution spectroscopy. J. Cohen acknowledges partial support from NSF grants AST-0507219 and AST0908139. I. Thompson acknowledges partial support from NSF AST-0507325. E.N.K. acknowledges support from the Southern California Center for Galaxy Evolution, a multicampus research program funded by the University of California Office of Research, and partial support from NSF grant AST-1009973. This work was partially supported by Sonderforschungsbereich SFB 881 "The Milky Way System" (subproject A4) of the German Research Foundation (DFG). We are grateful to the many people who have worked to make the Keck Telescopes and their instruments, and the Magellan Telescopes and their instruments, a reality and to operate and maintain these observatories. The authors wish to extend special thanks to those of Hawaiian ancestry on whose sacred mountain we are privileged to be guests. Without their generous hospitality, none of the observations presented herein would have been possible.

\section{APPENDIX}

\section{NOTES ON INDIVIDUAL STARS}

The hot turnoff region dwarf HE0102-0633 has very strong $\mathrm{Na} \mathrm{D}$ lines and a very strong resonance line of K I at $7699 \AA$. This star has a high reddening for a EMP star from the HES, $E(B-V)=0.14 \mathrm{mag}$, and $v_{r}=+2 \mathrm{~km} \mathrm{~s}^{-1}$. Hence we only derive an upper limit for $[\mathrm{Na} / \mathrm{Fe}]$ for this star. We believe these features are mostly interstellar in origin.

A similar situation appears to hold for much cooler star HE1416-1032. The detected features include strong NaD lines and an upper limit for the $5688 \AA \mathrm{Na}$ I line, but $v_{r}$ for this star is $\sim 0 \mathrm{~km} \mathrm{~s}^{-1}$, and $E(B-V)$ is also rather high for the present sample, 0.08 mag. One ISM component can be seen about $0.4 \AA$ to blue of each component of the $\mathrm{NaD}$ lines, and there may be other components within the feature we consider the stellar $\mathrm{NaD}$ lines. We consider the equivalent widths of all three $\mathrm{Na}$ I features as upper limits, and set the stellar $[\mathrm{Na} / \mathrm{Fe}]$ upper limit to the most stringent of these, which comes from the $5688 \AA$ line.

\section{REFERENCES}

Aihara, H., Allende Prieto, C., An, D., et al. 2011, ApJS, 193, 29 Andrievsky, S. M., Spite, F., Korotin, S. A., et al. 2011, A\&A, 530, A105 Andrievsky, S. M., Spite, M., Korotin, S. A., et al. 2010, A\&A, 509, 88 Aoki, W., Beers, T. C., Honda, S., \& Carollo, M. 2010, ApJ, 723, L201 Aoki, W., Norris, J. E., Ryan, S. G., Beers, T. C., \& Ando, H. 2002, ApJL, 576, L141

Asplund, M. 2005, ARA\&A, 43, 481

Asplund, M., Grevesse, N., Sauval, A. J., \& Scott, P. 2009, ARA\&A, 47, 481

Barklem, P. S., Christlieb, N., Beers, T. C., et al. 2005, A\&A, 439, 129

Baumüller, D. G., \& Gehren, T. 1997, A\&A, 325, 108

Beers, T. C., \& Christlieb, N. 2005, ARA\&A, 43, 531

Beers, T. C., Preston, G. W., \& Shectman, S. 1985, AJ, 90, 2089

Beers, T. C., Preston, G. W., \& Shectman, S. 1992, AJ, 103, 1987

Beers, T. C., Rossi, S., Norris, J. E., et al. 1998, SSRv, 84, 139

Bensby, T., Feltzing, S., Lundström, I., \& Ilyin, I. 2005, A\&A, 433, 185

Bergemann, M., \& Gehren, T. 2008, A\&A, 492, 823

Bergemann, M., Lind, K., Collet, R., Magic, Z., \& Asplund, M. 2012, MNRAS, 427, 27

Bernstein, R., Shectman, S. A., Gunnels, S. M., Mochnacki, S., \& Athey, A. E. 2003, Proc. SPIE, 4841, 1694

Bisterzo, S., Gallino, R., Straniero, O., Cristallo, S., \& Kappeler, F. 2011, MNRAS, 418, 284 
Bonifacio, P., Spite, M., Cayrel, R., et al. 2009, A\&A, 501, 519 Brown, J. A., Wallerstein, G., \& Zucker, D. 1997, AJ, 114, 180 Bullock, J., \& Johnston, K. V. 2005, ApJ, 635, 931

Busso, M., Gallino, R., \& Wasserburg, G. J. 1999, ARA\&A, 37, 239

Caffau, E., Bonifacio, P., François, P., et al. 2011, Natur, 477, 67

Carney, B. W., Wright, J. S., Sneden, C., et al. 1997, AJ, 114, 363

Carollo, D., Beers, T. C., Lee, Y. S., et al. 2007, Natur, 450, 1020

Carretta, E., Gratton, R., Cohen, J. G., Beers, T. C., \& Christlieb, N. 2002, AJ, 124,481

Castelli, F., \& Kurucz, R. L. 2004, arXiv:astro-ph/0405087

Cayrel, R., Depagne, E., Spite, M., et al. 2004, A\&A, 416, 1117

Christlieb, N., Bessell, M. S., Beers, T. C., et al. 2002, Natur, 419, 904

Christlieb, N., Schörck, T., Frebel, A., et al. 2008, A\&A, 484, 721

Cohen, J. G. 2004, AJ, 127, 1545

Cohen, J. G., Christlieb, N., Beers, T. C., Gratton, R., \& Carretta, E. 2002, AJ, 124,470

Cohen, J. G., Christlieb, N., McWilliam, A., et al. 2004, ApJ, 612, 1107

Cohen, J. G., Christlieb, N., McWilliam, A., et al. 2008, ApJ, 672, 320

Cohen, J. G., Christlieb, N., Qian, Y., \& Wasserburg, G. 2003, ApJ, 588, 1082

Cohen, J. G., Christlieb, N., Thompson, I., McWilliam, A., \& Shectman, S 2012, in ASP Conf. Ser. 458, Galactic Archeology: Near-Field Cosmology and the Formation of the Milky Way, ed. W. Aoki, M. Ishigaki, T. Suda, T. Tsuijimoto, \& N. Arimoto (San Francisco, CA: ASP), 61

Cohen, J. G., \& Huang, W. 2009, ApJ, 701, 1053

Cohen, J. G., \& Kirby, E. 2012, ApJ, 760, 86

Cohen, J. G., McWilliam, A., Christlieb, N., et al. 2007, ApJL, 659, L161

Cohen, J. G., McWilliam, A., Shectman, S., et al. 2006, AJ, 132, 137

Cohen, J. G., \& Melendez, J. 2005, AJ, 129, 303

Cohen, J. G., Shectman, S., Thompson, I., et al. 2005, ApJL, 633, L109

Cooke, R., Pettini, M., \& Murphy, M. T. 2012, MNRAS, 425, 347

Cutri, R. M., et al. 2003, Explanatory Supplement to the 2MASS All-Sky Data

Release, http://www.ipac.caltech.edu/2mass/releases/allsky/doc/explsup.html de Jong, J. T. A., Yanny, B., Rix, H. W., et al. 2010, ApJ, 714, 663

Depagne, E., Hill, V., Spite, M., et al. 2002, A\&A, 390, 187

Dobrovolskas, V., Kucinskas, A., Andrievsky, S. M., et al. 2012, A\&A, 540, A 128

Dominy, J. F. 1984, ApJS, 55, 27

Edvardsson, B., Andersen, J., Gustaffson, B., et al. 1993, A\&A, 275, 101

Francois, P., Depagne, E., Hill, V., et al. 2008, A\&A, 476, 935

Frebel, A., Aoki, W., Christlieb, N., et al. 2005, Natur, 434, 871

Frebel, A., Christlieb, N., Norris, J. E., et al. 2006, ApJ, 652, 1585

Frebel, A., Norris, J. E., Aoki, W., et al. 2007, ApJ, 658, 534

Fulbright, J. P. 2002, AJ, 123, 404

Fulbright, J., Rich, R. M., \& Castro, S. 2004, ApJ, 612, 447

Fumagalli, M., O’Meara, J. M., \& Prochaska, J. X. 2011, Sci, 334, 1245

Gratton, R. G., Carretta, E., Desidera, S., et al. 2003, A\&A, 406, 131

Gratton, R., Sneden, C., \& Carretta, E. 2004, ARA\&A, 42, 385

Greggio, L., Renzini, A., \& Daddi, E. 2008, MNRAS, 388, 829

Grevesse, N., \& Sauval, A. J. 1998, SSRv, 85, 161

Gunn, J. E., \& Griffin, R. F. 1979, AJ, 84, 752

Gustaffson, B., Bell, R. A., Eriksson, K., \& Nordlund, B. 1975, A\&A, 42, 407

Hansen, C. J., Bergemann, M., Cescutti, G., et al. 2013, A\&A, 551, A57

Heger, A., \& Woosley, S. E. 2010, ApJ, 724, 341

Honda, S., Aoki, W., Beers, T. C., \& Takada-Hidei, M. 2011, ApJ, 730, A77

Houdashelt, M. L., Bell, R. A., \& Sweigart, A. V. 2000, AJ, 119, 1448

Ivanova, D. V., \& Shimanskiı̌, V. V. 2000, ARep, 44, 376

Ivans, I. I., Sneden, C., Renee James, C., et al. 2003, ApJ, 592, 906

Iwamoto, K., Brachwitz, F., Nomoto, I. I., et al. 1999, ApJS, 125, 439

Izzard, R. G., Glebbeek, E., Stancliffe, R. J., \& Pols, O. 2009, A\&A, 508, 1359

Johnson, J. A., \& Bolte, M. 2002, ApJL, 579, L87

Kelson, D. D. 2003, PASP, 115, 688

Kim, Y.-C., Demarque, P., Yi, S. K., \& Alexander, D. R. 2002, ApJS, 143, 499

Kirby, E. N., Cohen, J. G., Smith, G. H., et al. 2011, ApJ, 727, 79

Kobayashi, C., Umeda, K., Nomoto, K., Tominaga, N., \& Ohkubo, T. 2006, ApJ, 653,1145

Kurucz, R. L. 1993, ATLAS9 Stellar Atmosphere Programs and 2 km/s Grid, (Kurucz CD-ROM No. 13)

Lai, D., Rockosi, C. M., Bolte, M., et al. 2009, ApJL, 697, L63

Latham, D. W., Stefanik, R. P., Torres, G., et al. 2002, AJ, 124, 1144

Lau, H., Stancliffe, R. J., \& Tout, C. A. 2007, MNRAS, 385, 301

Lee, Y. S., Beers, T. C., Sivarani, T., et al. 2008, AJ, 136, 2022

Lind, K., Asplund, M., Barklem, P. S., \& Belyaev, A. K. 2011, A\&A, 528, A103

Lucatello, S., Beers, T. C., Christlieb, N., et al. 2006, ApJ, 652, L37
Lucatello, S., Gratton, R., Cohen, J. G., et al. 2003, AJ, 125, 875

Lucatello, S., Tsangarides, S., Beers, T. C., et al. 2005, ApJ, 625, 825

Lugaro, M., Karakas, A. I., Stancliffe, R. J., \& Rijs, C. 2012, ApJ, 747, A2

Magic, Z., Collet, R., Hayek, W., \& Asplund, M. 2013, A\&A, submitted (arXiv:1307.3273)

McCarthy, J. K. 1988, PhD thesis, California Institute of Technology

McClure, R. D. 1997, PASP, 109, 256

McWilliam, A., Preston, G. W., Sneden, C., \& Searle, L. 1995a, AJ, 109, 2757

McWilliam, A., Preston, G. W., Sneden, C., \& Shectman, S. 1995b, AJ, 109,2736

Nissen, P. E., \& Schuster, W. J. 2010, A\&A, 511, L10

Nissen, P. E., \& Schuster, W. J. 2011, A\&A, 530, A15

Nomoto, K., Thielemnn, F. K., \& Yokoi, K. 1984, ApJ, 286, 644

Nomoto, K., Tominaga, N., Umeda, H., Kobayashi, C., \& Maeda, K. 2006, NuPhA, 777, 424

Norris, J. E., Bessell, M. S., Yong, D., et al. 2013a, ApJ, 762, A25

Norris, J. E., Christlieb, N., Korn, A. J., et al. 2007, ApJ, 670, 774

Norris, J. E., Yong, D., Bessell, M. S., et al. 2013b, ApJ, 762, A28

Oke, J. B., \& Gunn, J. E. 1982, PASP, 94, 586

Preston, G. W., \& Sneden, C. 2001, AJ, 122, 1545

Preston, G. W., Sneden, C., Thompson, I. B., Shectman, S. A., \& Burley, G. S. 2006, AJ, 132, 85

Qian, Y. Z., \& Wasserburg, G. J. 2008, ApJ, 687, 272

Rafelski, M., Wolfe, A., Prochaska, J. X., Neeleman, M., \& Mendez, A. J. 2012, ApJ, 755, A89

Ralchencko, Yu., Kramida, A. E., Reader, J., \& the NIST ASD Team. 2010, NIST Atomic Spectra Database (version 4.0), http://physics.nist.gov/asd

Ramírez, S. V., Cohen, J. G., Buss, J., \& Briley, M. M. 2001, AJ, 122, 1429

Reddy, B. E., Tomkin, J., Lambert, D. L., \& Allende Prieto, C. 2006, MNRAS, 367,1329

Roederer, I. U. 2009, AJ, 137, 272

Roederer, I. U., Sneden, C., Lawler, J. W., \& Cowan, J. J. 2010, ApJL, 714, L123

Schlaufman, K. C., Rockosi, C. M., Lee, Y. S., et al. 2012, ApJ, 749, A77

Schlegel, D. J., Finkbeiner, D. P., \& Davis, M. 1998, ApJ, 500, 525

Schörck, T., Christlieb, N., Cohen, J. G., et al. 2009, A\&A, 507, 817

Schuster, W. J., Moreno, E., Nissen, P. E., \& Pichardo, B. 2012, A\&A, 538, A21

Sesar, B., Juric, M., \& Ivezic, Z. 2011, ApJ, 731, 4

Shi, J. R., Gehren, T., Mashonkina, L., \& Zhao, G. 2009, A\&A, 503, 533

Shortridge, K. 1993, in ASP Conf. Ser. 52, Astronomical Data Analysis Software and Systems II, ed. R. J. Hannisch, R. J. V. Brissenden, \& J. Barnes (San Francisco, CA: ASP), 21

Skrutskie, M. F., Cutri, R. M., Stiening, R., et al. 2006, AJ, 131, 1163

Smith, J. A., Tucker, D. L., Kent, S., et al. 2002, AJ, 123, 2121

Sneden, C. 1973, PhD thesis, Univ. of Texas

Sneden, C., Cowan, J. J., Lawler, J. E., et al. 2003, ApJ, 591, 936

Sneden, C., Lawler, J. E., Cowan, J. J., Ivans, I. I., \& Den Hartog, E. A. 2009, ApJS, 182, 80

Sobeck, J. S., Kraft, R. P., Sneden, C., et al. 2011, AJ, 141, 175

Spite, M., Andrievsky, S. M., Spite, F., et al. 2012, A\&A, 541, A143

Spite, M., Cayrel, R., Hill, V., et al. 2006, A\&A, 455, 291

Stephens, A., \& Boesgaard, A. M. 2002, AJ, 123, 1647

Takeda, Y., Zhao, G., Chen, Y. Q., Qui, H. M., \& Takada-Hidai, M. 2002, PASJ, 54,275

Thevenin, F. \& Idiart, T. P. 1999, ApJ, 521, 753

Tissera, P. B., White, S. D. M., \& Scannapieco, C. 2012, MNRAS, 420, 255

Tolstoy, E., Hill, V., \& Tosi, M. 2009, ARA\&A, 47, 371

Tominaga, N., Umeda, H., \& Nomoto, K. 2007, ApJ, 660, 516

Travaglio, C. D., Gallino, R., Arnone, E., et al. 2004a, ApJ, 601, 864

Travaglio, C., Hillebrandt, W., Reinicke, M., \& Thielemann, F. K. 2004b, A\&A, 425,1029

Tsangarides, S., Ryan, S. G., \& Beers, T. C. 2004, MmSAI, 75, 772

VandenBerg, D. A., Bergbusch, P. A., Dotter, A., et al. 2012, ApJ, 755, A15

Ventura, P., \& d'Antona, F. 2009, A\&A, 499, 835

Vogt, S. E., Allen, S. L., Bigelow, B. C., et al. 1994, Proc. SPIE, 2198, 362

Wallerstein, G., \& Knapp, G. R. 1998, ARA\&A, 36, 369

Wisotzki, L., Christlieb, N., Bade, N., et al. 2000, A\&A, 358, 77

Woosley, S. E., \& Weaver, T. A. 1995, ApJS, 101, 181

Yi, S., Kim, Y.-C., \& Demarque, P. 2003, ApJS, 144, 259

Yong, D., Norris, J. E., Bessell, M. S., et al. 2013, ApJ, 762, A26

York, D. G., Adelman, J., Anderson, J. E., Jr., et al. 2000, AJ, 120, 1579

Zhang, L., Karlsson, T., Christlieb, N., et al. 2011, A\&A, 528, A92

Zolotov, A., Willman, B., Brooks, A., et al. 2010, ApJ, 721, 783 